\title{
Estudo da Existência da Fase de Kosterlitz-Thouless no Estado Fundamental de Rotores Quânticos
}

OSCAR BOLINA JƯNIOR

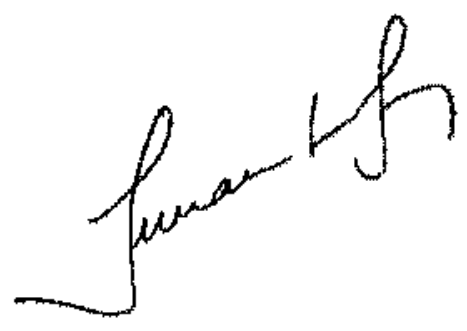

Tese de doutorado apresentada ao Instituto de Física da

Universidade de Sầo Paulo

Orientador: Prof. Dr. Jose Fernando Perez

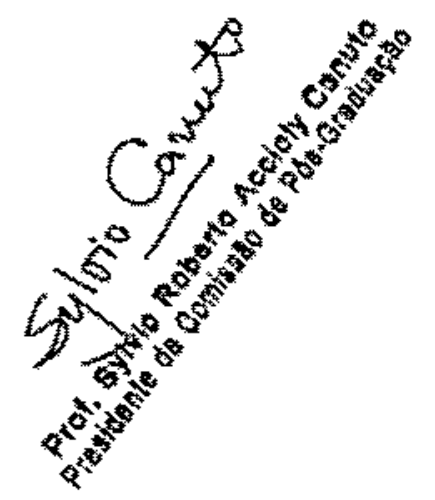

Dezembro, 199 ?

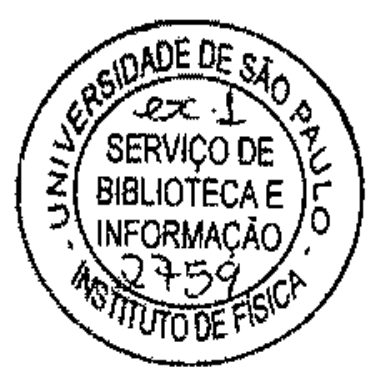

Prof. Dr. José Fernando Perez - IFUSP (Orientador)

Prof. Dr. Walter Wreszinski - IFUSP

Prof. Dr. Eduardo Jordão Neves - IME

Ptof. Dr. Clodoaldo Grotta Ragazzo- IME

Prof. Dr. Bruto Max Pimentel Escobar - IFT 


$$
\begin{gathered}
530.414 \\
B 689 \\
D \\
8 x .1
\end{gathered}
$$

\section{FICHA CATALOGRÁFICA}

Preparada pelo Serviço de Biblioteca e Informaçăo do Instituto de Física da Universidade de Săo Paulo

\section{Bolina Junior, Oscar}

Estudo da Existência da Fase de Kosterlitz-Thouless no Estado Fundamental de Rotores Quânticos. Såo Paulo, 1997.

Tese (Doutoramento) Universidade de \$ão Paulo. Instituto de Fisica - Departamento de Física Matemática

Area de Concentraçăo: Fisica das Partículas Elementares Orientador: Prof. Dr. José Femando Perez

Unitemos: 1. Transição de Kosterlitz-Thouless;

2. Rotores Quânticos; 3. Estimativas de Correlaçäo;

4. Representação das Cargas; 5. Transformaçẫo de Sine-Gordon. 


\title{
RESUMO
}

Investigamos a existência de uma fase de Kosterlitz-Thouless no estado fundamental de um sistema de rotores quânticos em uma dimensăo. Provamos que o modelo nảo exibe uma transiçăo de primeira ordem, já que a estimativa de McBryan-Spencer é válida para os rotores. Obtemos a funçăo de partição do modelo nas representaçoes de Lie-Trotter, de sine-Gordon, e na representaçäo das cargas. Nessa última, provamos um limite inferior paza a função de correlaçāo entre cargas externas.

Ainda na representaçâo das cargas, damos uma nova prova do decaimento polinomial do limite superior para a função de correlação de um gás de dipolos (caroço duro) na presença de cargas externas.

\begin{abstract}
We investigate the existence of a Kosterlitz-Thouless phase in the ground state of a one-dimensional array of quantum rotators. We prove that this model does not exhibit a first-order phase transition since a McBryan-Spencer bound holds for it. We obtain the partition function of the rotators in the Lie-Trotter, sine-Gordon, and charge representations. In this latter representation, we give a new proof of the upper bound polynomial decay on the external charges correlation function of a dipole gas with hardcore.
\end{abstract}




\section{AGRADECIMENTOS}

Mais uma vez me cabe agradecer ao Prof. Perez já tantos anos de orientaçäo e troca de idéias (de fato, näo é bem troca, visto que elas vinham mais de lá para cá). A par do trabalho científico, fique registrada minha gratidão por seu empenho naqueles sábados fora de hora, naqueles dias de imigrante ilegal , ...;

Ao Parreira, com cuja colaboraçăo esta tese (e já alguns papers) foi elaborada;

Ao Mingo, que tem me ajudado a resolver o que deixo sem resposta aqui;

Ao Departamento de Física-Matemática pelo suporte administrativo;

Sou grato ao Prof. Iberê Caldass por sua ajuda e paciência na conclusão deste trabalho;

Muito especialmente agradeço ao Prof. Abel Klein, da Universidade da Califótina, Irvine, que por um ano tăo generosamente aí me receben;

A FAPESP e à CAPES, o apoio financeiro. 


\section{Conteúdo}

1 Introduçào à Transição de Kosterlitz-Thouless 13

1.1 Thansiçães de Segunda Ordem ............... 16

1.2 A Transição de Kosterlitz-Thouless . . . . . . . . . . . . 17

1.3 A Transição em Gases de Coulomb . . . . . . . . . . . 19

2 Revisäo do Gás de Coulomb e Alguns Resultados 21

2.1 Gases de Coulomb ................... 21

2.2 A Representaçāo de sine-Gordon do Gás de Coulonb ..... 24

2.3 A Função de Correlação . . . . . . . . . . . 26

2.4 Limite Inferior da Função de Correlação . . . . . . . . 26

2.5 A Função de Partição de Cargas Externas . . . . . . . . . 27

2.6 O Operador de Desordem . . . . . . . . . . 28

3 Estimativas de Correlação na Representação das Cargas 30

3.1 Introdução . . . . . . . . . . . . . 30

3.2 A Função de Partição de Gases de Dipolos . . . . . . . . . 32

3.3 A Desigualdade Eletrostática . . . . . . . . . . 33

3.4 As Cargas Externas . . . . . . . . . . . . . . . . 34

3.5 A Expansão de Cluster . . . . . . . . . . . . . . . 36

3.6 O Limite Superior . . . . . . . . . . . . . . . . 37

3.7 Dipolos de Comprimentos Diferentes ... . . . . . . 39

3.8 Renormalizaçâo em $L_{1}$ e $L_{2} \ldots \ldots \ldots \ldots \ldots \ldots$

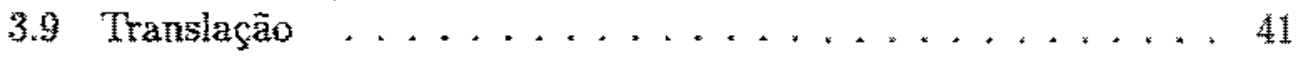

3.10 Expansōes de Cluster . . . . . . . . . . . . . . 42

4 Modelo de Villain 45

4.1 Combinaçoes Convexas . . . . . . . . . . . 46 
4.2 A Construção dos Blocos $\ldots \ldots \ldots \ldots \ldots \ldots, \ldots \ldots$

4.3 Reprimindo a Atividade $\ldots \ldots \ldots \ldots \ldots \ldots$

4.4 Um Passo Intermediário $\ldots \ldots \ldots \ldots \ldots \ldots$

4.5 O Procedimento Indutivo para a Função de Partição .... 52

4.6 A Hipótese Indutiva . . . . . . . . . . . . 54

4.7 Extração da Energia de Cargas Isoladas . . . . . .

4.8 A Função de Correlaçäo das Cargas Externas ....... 58

5 Rotores Quânticos 61

5.1 A Função de Partiçāo . . . . . . . . . . . . 61

5.2 Exemplos de Rotores Planos . . . . . . . . . . 65

5.3 A Aproximação de Villain . . . . . . . . . . . . 66

5.4 A Estimativa de McBryan-Spencer . . . . . . . . . . . 69

6 A Transformação de Dualidade 72

6.1 Transformada de Fourier . . . . . . . . . . . 72

6.2 O Modelo Dual de Villain e a Representaçäo das Cargas ... 75

6.3 Estimativa da Correlą̧äo entre Cargas Externas . . . . . 76

6.4 O Operador de Desurdem . . . . . . . . . . 77

6.4 .1 Comentários. .................. 81

6.5 Conclusão ................. 83

A. A Desigualdade Eletrostätica

B A Função de Green 86 


\section{APRESENTAÇĀO}

Nesta tese estudamos um sistema de rotores quânticos em uma rede unidimensional com vistas a demonstrar a existência de uma fase de KosterlitzThouless para o estado fundamental desse modelo no limite termodinâmico de volume infinito.

O sistema de rotores que estudamos ô descrito pelo Hamiltoniano

$$
H=\sum_{x} \frac{p^{2}(x)}{2 I}-J \sum_{x} \cos (\theta(x)-b(x+1))
$$

$x \in \mathrm{Z}$, com acoplamento ferromagnético, $J>0$, onde $\theta(x) \in[-\pi, \pi]$ é a co-

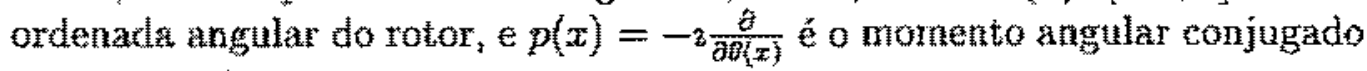
à variátyel $\theta(x)$.

A idéia de demonstrar a transição de Kosterlitz-Thouless para esse modelo parece natural se lembramos que alguns modelos clássicos bidimensionais na rede como o rotor plano, o gás de Villain, e o gás de caroço duro apresentam uma fase de Kosterlitz-Thouless abaixo de certo valor crítico da temperatura, no qual a função de correlação tem apenas decainnento polinomial, com um expoente que depende da temperatura.

Os rotores quânticos untidimensionais podem ser mapeados, por meio da representação de Lie-Trotter, num sistema classsico bidimensional. O mapeamento produz um gás de Villain semelhante ao clássico com interą̧āo coulombitua em duas dimensōes.

A diferençà estat em que no sistema clássico bidimensional en que o modelo quântico paçamento depende de un paràmetro discreto $n$ que no fim dever ser tomado no limite $n \rightarrow \infty$. Como o valor do espaçamento è $n^{-1}$, no limite $n \rightarrow \infty$ obtemos um contínuo nessa direçāo.

A idéia básica da tese é aplicar o mesmo método de prova da transição Kosterlitz-Thouless que foi usado com sucesso nos modelos clássicos citados acima para o gás de Villain na rede anisotrópica, com obtenção de resultados no limite do contínuo na direçäo temporal extra.

Vale lembrar ainda que gases de Coulomb no contínuo também exibem uma 
fase de Kosterlitz-Thouless, a qual pode ser provada usando essencialmente o mesmo procedimento empregado na rede.

Loygo, o modelo de rotores, que leva a um gás na rede com espaçamento discreto em uma direçâo, contínuo na outra, não deve -- em princípio - apresentar comportamento muito diferente do correspondente gás na rede discreta ou no contíno. O problema é fazer o discreto terder ao conthou corretamente. Provar a transiçăo de Kosterlitz-Thouless significa mostrar que a função de corralação do modelo tem dois comportamentos distintos em funçäo da temperatura. Especificamente, a transição de Kosterlitz-Thouless é a passagem de uma fase de alta temperatura em que 0 decaimento da correlaçäo é exponencial a um lase de baixa temperatura em que o decaimento da correlação é polinomial.

Nos rotores, $\odot$ acoplamento $J$ substitui a temperatura (ou temperatura inversa b) como paràmetro de determinação das fases do modelo. A idéia é mostrar que ordem de longo alcance existe para valores suficientemente grandes de $J$. Além disso, nossas estimativas devem ser uniformes em $n$, ou seja, independentes desse parâmetro no limite do contínuo.

No entanto, encontramos dificuldades técnicas na execuçäo desse roteiro; o que nos tem impedido de concluir a prova. Conseguimos escrever a função de partiçăo gás de Villain resultante do mapeamento dos rotores à semelhança de urn gás de Villain clássico na rede bidimensional. Desenvolvemos todos as elementos da teoria da transição de Kosterlitz-Thouless de gases clássicos para o nosso gás de Villain, como representaçăo de sine-Gordon, operador de desordem, espaço dual; obtemos estimativas de correlaçăo, como a estimativa de McBryan-Spencer e de Jensen. A referência basica atqui ainda é [24], [25].

No entanto, o método de prova da transiçăo aplicado à função de partiçăo do gás de Villain obtido a partir dos rotores năo se mostrou adequado para lidar com o parämetro discreto do eixo temporal. No caso de gases clássicos, a prova segue por indução sobre escalas de blocos construídos na rede em que, em cada escala, o balanço energia-entropia nos blocos dever ser favorável, no sentido de que a energia ganha da entropia. Estarnos convencidos de que para - gás de Villain que obtivemos esse balanço é favorável a partir de uma escala grande, na qual os blocos têm lados-digamos - macroscópicos, qualquer coisa independente de $n$. O problema é que para se chegar até essa escala por induçã a, é preciso acertar o balanço energia-entropia em alguma escala inicial menor, em que o espaçamento de rede desequilibria aquele balanço em 
favor da entropia.

É irónico encontrarmos dificuldade com os rotores, una vez que esse sistema tếm servido de base para a explicaçăo heuritica da transiçäo de KosterlitzThouless [20].

Os resultados de correlação a que aludimos acima mostram que os rotores năo possuem transiçăo de primeira ordem, mas nada nos dizem acerca de transiçöes de ordens mais altas, como é à de Kosterlitz-Thouless.

Esta tese é essencialmente um trabalho de desenvolvimento de métodos para obtenção de estimativas de correlaçâo tanto para gases clássicos como para gases obtidos por mapeamento do sistema quẫntico.

Ja desde o início da tese nos propusemos a verificar como certas estimativas de correlação, mesmo em modelos clássicos para os quais a transiçẵo de Kosterlitz-Thouless já havia sido provada, podia ser feita diretamente na representação das cargas. Como se verá adiante, a chamada representaçäo de sine-Gordon é o instrumento de preferência para a realização de tais provas. O método de obter estimativas de correlaçäo que apresentamos em conexão com a representação das cargas consiste em escrever a função de correlação em expansão de cluster. As dificuldades com essas expansoes sằ geralmente muito grandes. Todavia, no caso da correlaçäo, a expansão de cluster, combinada com a desigualdade eletrostática, traz grande simplificidade.

O elemento de relevância aqui é que muitos modelos não possuem uma transformaçăo de sine-Gordon diponivel, e entâo näo há como dispensar a representaçäo das cargas para efetuar as estimativas.

Sumarizamos nossos resultados abaixo, juntamente com a descriçäo da tese,

O Capítulo 1 é dedicado a uma introduçào bastante geral à teoria das transiçoes de fase, começando pelas transiçóes de fase mais simples e conliecidas, que são as da água ao passar de um estado físico a outro. Apresentamos a idéia do ordem-desordem e de parâmetro de ordem ainda no ambito das transiçöes de primeira ordem.

A seguir, discutimos brevemente alguns resultados relacionados com transiçōes de segunda orden para entrarmos no tema principal da transição de Kosterlitz-Thouless em sistemas bidimensionais.

No Capítulo 2 fazzemos uma revisão de gases de Coulomb e enunciamos os resultados acerca da transiçấo de Kosterlitz-Thouless nesses sistemas. Explicamos o que é a representaçâa de sine-Gordon, e como esses resultados 
săo conseguidos nessa representação. Para isso, definimos a funçäo de cor* relaçäo e o operador de desordem para os gases de Coulomb. Discutimos como a obtençẵo de um limite inferior para a função de correlaçào, através da desigualdade de Jensen, é o primeiro passo para provar a existência da transiçāo de Kosterlitz-Thouless. O passo seguinte é encontrar um limite superior para a funçăo de correlaçāo (ou, o que é equivalente, um limite inferior para o operador de desordem).

Esses limites - e, en particular, o limite superior para a funģăo de correlaçăo - são usualmente obtidos na representaçâo de sine-Cordon. Para gases de dipolos e de Coulomb o limite superior para a correlação foi provado por Frohlich-Spencer [22] nessa representaçào.

Não conhecemos nenhuma prova alternativa desses resultados que tenha sido feita diretamente na representação das cargas. Os próprios autores dessas provas comentam [24]:

If one attempts to obtain such results (estimativas de correlaçāo) directly in the $q$ representation the required estimates appear to be far more complicated.

O leitor poderá ver no Capítulo 3 como pudemos obter limites superiores para a função de correlaçäo de um gás de dipolos de orientaçăo discreta que não se sobrepöem, sem usar a representaçäo de sine-Gordon. Fizemos isso primeiramente para o caso mais simples de um gás de dipolos de comprimentos iguais, e depois generalizamos para o caso em os dipolos possuem dois comprimentos diferentes. Conseguimos sscrever uma expansäo de cluster para a funçầo de correlaçẫo fácil de trabathar. Os termos mais dificeis sẵo postos numa forma convariente para derivar o resultado que desejamos. Pelas perspectivas que abre de poder ser empregado para sistema que não possuem uma transformação de sine-Gordon disponivel, achamos que esse método é a principal contribuição da tese. Provamos então:

Teorema 1. A função de correlação de um gás de dipolos com caroço duro é limitada superiormente por

$$
G(x, y) \leq C_{z} e^{-m \log |x-y|}
$$


$\operatorname{com} C_{p}<\infty e$

$$
m=\frac{\beta}{2 \pi}\left(1+(1+\epsilon) \beta z e^{-(3) 16}\right)^{-1}
$$

para qualquer $t>0$, onde 0 é a temperatrura inversa, $z$ e a atividade.

Estimativa sernelhante vale para dipolos de comprimentos diferentes.

Convum citar que uma representaço das carghs associada com modelos de percolaçấ foi usada em [34].

Como tratamos somente de dipolos no capítulo anterior, achamos por bem apresentar o método já bem conhecido de obtenção de limites superiores para a funçào de correlação usando a representação de sinte-Gordon. Isso é feito no Capítulo 4, para o gás de Villain. Procuramos simplíficar ao máxírno a prova, sem nos preocupar com a otimizaçäo das estimativas. Convém lembrar, no entanto, que todos os elementos dessa prova - que é muito mais elaborada do que para os dipolos - já estão contidos no tratamento dos di. polos (comprimentos diferentes) do capítulo anterior. Esperamos que o uso combinado desses dois capítulos - ainda que empregando métodos diferentes - possa levar a um entendimento dos detalhes mais dificeis da transiçäo de Kosterlitz-Thouless.

No Capítulo 5 estudamos os rotores quânticos (0.1). Definimos nosso nodelo de rotores de modo bem geral e empregamos a formula de Trotter para obter fungäo de particăo do modelo dássico bitimensional no qual os roto* res loram mapeados. O mapeamento levon a

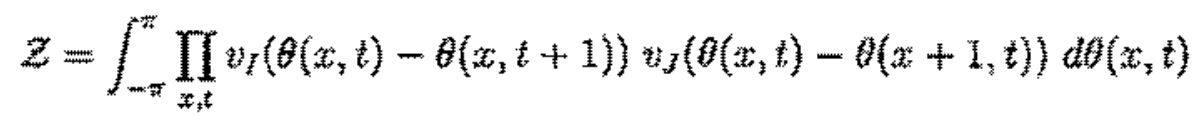

comer

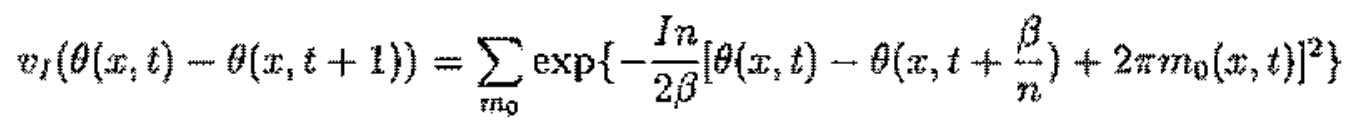

e

$$
v_{J}(\theta(x, t)-\theta(x+1, t))=\exp \left\{-\frac{\beta J}{n} \cos (\theta(x, t)-\theta(x+1, t))\right\}
$$

Nessa representaçẫo deduzimos uma estimativa de McBryan-Spencer para a funçấo de correlaçāo dos rotores quânticos na chamada aproximaçăo de Villain para o potencial da forma co-seno dada acima. Tal aproximaçäo 
consiste em substituir o potencial en (0.2) por una gaussiana periodizada. Assim, em vez do comseno, a nova interaçäo é lada por

$$
\sum_{m=-\infty}^{\infty} e^{-(z / 2)\left(0_{i}-\theta_{j}+2 \pi m\right)^{2}}
$$

A estimativa de MeBryan-Spencer é uma garantia da nëo exisyencia de transiç艹 de ordens mais altats.

Temos

Teorema 2. Seja $G\left(y-y^{\prime}: s-s^{\prime}\right)$ a função da correlaçăo entre dois sítios $(y, s),\left(y^{\prime}, s^{\prime}\right)$ na rede bidimensional no limite termodinâmico de $\beta, n \rightarrow \infty$. Temos a seguinte estimativa

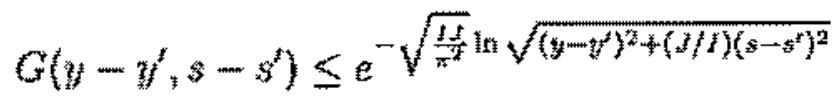

No Capítulo 6, escrevemos a funça de partiçăo do sistema na representaçäo de sine-Gordon. Isso é feito por meio de uma transformaçāo de dualidade - que é o efetuar a transformata de Fourier na variuvel $\theta$ do integrando da função de partiçăo obtida acima.

Nessa representação a função de partiçäo dos totores se escreve

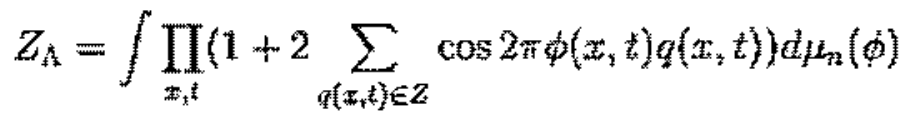

onde

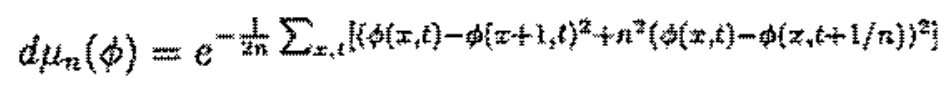

é a gaussiana discreta.

A partir daqui, a prova da transiçāo de Kosterlitz-Thouless seguiria - em princípio - pelas expansöes de multi-escala do gás obtido como foi feito para o gás de Coulomb em [30].

Como dissemos, temos encontrado dificuldades téchicas para realizaçäo desse programa. No final do Capitulo 6 tecemos comentários sobre o metodo de construção de blocos e extraçāo da auto-entergia desses blocos com o qual 
se reprime a atividade do gás e se obtém estimativas válidas quando a atividade é pequena. Em nossa rede de espaçamentos desiguais, no limite do contínuo do espaçamento em uma única direção, o balanço entre agrupamento de blocos e extraçäo da auto-energia mostra-se desfavorável a que façamos a atividade pequena e impede a obtençăo de estimativas de correlaçăo.

Da representação de sine-Gordon, passamos a representação das cargas, e aí estabelecenos um límite inferior para a funçäo de correlação entre cargas externas do gás de Villain resultante:

Teorema 3. Seja a funçäo de correlação de cargas externas

$$
Z^{E}(x)=Z^{-1} \int e^{-(1 / 2)\left(q_{A}+p(y, t), \cdots \Delta^{-1}\left(y_{\lambda}+\rho(y, t)\right)\right)} \prod_{y, t} d \lambda q(y, t)
$$

onde $\mathcal{Z}$ a a funçăo de partição na ausência de cargas externas, e pax a distribuiçảo das cargas externas fracionánas colocadas no sistema, uma $+\xi$, situada na origem, outra, $-\xi$, situada em $x$. Temos

$$
G_{A}^{\varepsilon}(x) \geq C_{\xi} e^{-\xi^{z} \sqrt{\operatorname{la}_{y}}|x| x \mid}
$$

$\operatorname{com} 0<C_{\xi}<\infty$

No Apèndice A mostramos como a desígualdade eletrostâtica, que é um instrumento básico de aplicaça nos capitulos 3,4 e 6 , como um exercício simples de completar quadrados no peso de Gibbs da medida do gás.

No Apêndice $B$ achamos por bem derivar explicitamente o comportamento assintótjeo da funçăo de Green do gás bidimensional obtido pelo mapeamento dos rotores, já que nossos resultados (Teoremas 2,3 ) se baseiatm na diferença $C(x, t)-C(0,0)$. Partindo da funçẫo de Green na rede finita com espaçamentos desiguais, mostramos como obter sua expressão no limite de volume infinito, espaçamento zero em uma direção, e posterior limite termodinâmico de temperatura zero. 


\section{Capítulo 1}

\section{Introdução à Transição de Kosterlitz-Thouless}

As transições de fase mais familiáres são as transiçôes entre gelo e água e ảgua e vapor. Essas transições enwolvem uma resstruturação microscópica da substância ao passar de uma fase à outra, e são chamadas transições de primeira ordem. No diagrama de fase de un liquido simples - por exemplo, no plano pressão $\times$ temperatura, como na figura acima - as linhas separaram os diferentes estados em que o líquido pode se apresentar. Cada linha, por sua vez, indica regióes de coexistência de fases. As fases sólida e gasosa estão em equilibrio ao longo dá curva de aublimaçán ; a curva de fusão é a de equilibrio entre as fases sólida e liquida: as fases líquida e gasosa estão em equilibrio ao longo da curva de pressäa de vapor. Essa última curva, em particular, termina $\mathrm{em}$ um ponto chamado ponto crítico, no qual o líquido pode ser convertido em gás contimuamente.

As transiçōes de primeira ordem ocorrem quando cruzamos as linhas de uma região à outra, ou de um estado a outro. Se observarmos a comportamento de uma grandeza do sistema - por exemplo a densidade - notaremos que seu valor dá um salto quando cruzamos as linhas que distinguem as fases.

Em contraste, há transiçōes na qual, ao cruzarmos as linhas de fase, a densidade (nosso exemplo de novo) permanece contínua. As grandezas que se alteram descontinuamente estâo relacionadas com as derivadas primeiras de algum potencial termodinâmico, que serầ descontínuas para valores de $P \ell$ T na curva. Essas transiçôes nầo săo de primeira ordem. Vamos chamar toda transição que não for de primeira ordem de transiçăo de segunda ordem. 


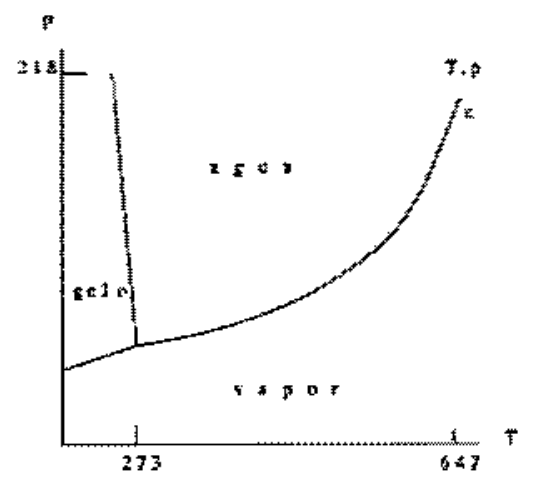

Figura 1.1: Diagrama de fase da água no plano $p T$ mostrando o ponto crítico em $c$

A idéia lundamental no estudo de transiçöes de fase é a de que a transiçăo pode ser deserita por um parâmetro de ordem. Já no àmbito das transiçôes de primeira ordem, é possível introduzir um parẫnetro de ordem associado com um grupo de simetria, seja ele finito, discreto ou contínuo. O parâmetro de ordem é uma medida do tipo de ordem existente nas vizinhangas de um ponto crítico. Por exemplo,

(i) num cristal ferromagnético, com um eixo de magnetização ao longo da direçăo $z$, um parâmetro de ordem conveniente é a média estatística da com ponente $z$ da magnetizaçâo em determinado ponto da rede. $O$ vetor magnetizagăo nào tem módulo determinado quando o campo aplicado ế nulo, podendo com isso apontar em direçoes diferentes. Essas escolhas de orientaçăo năo são mais possiveis, porém, quando a temperatura é maior que uma temperatura crítica, chamada temperatura de Curie;

(ii) no modelo de Ising, como parâmetro conveniente considera-se o valor esperado da interaçâo spin-spin $\left\langle\sigma_{j} \sigma_{j}\right\rangle$ entre spins situados em $i$ ej. Essas correlaçôes de longo alcance entre spins estão associadas com a ordem de longo alcance na qual a rede possui magnetizaçăo, mesmo na ausência de urn campo magnético. Tais transições magnéticas sẫo análogas à transiçāo líquido-gấs;

(iii) na transição líquido-gás, o parâmetro de ordem é dado pela diferenç̧a $\rho_{L}-\rho_{C}$ entre as densidades das fases coexistentes, ou seja, no estado líquido e gasoso. Aqui também há uma oscilação do valores da densidade quando a temperatura está abaixo da temperatura crítica. Tomando como exemplo 


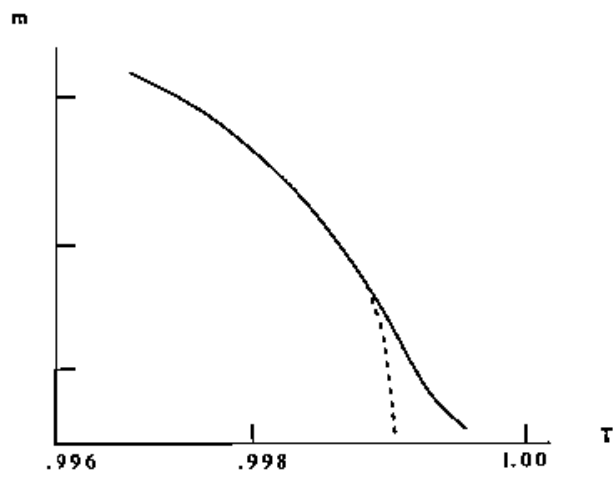

Figura 1.2: Diagrama do comportamento dos parâmetros de ordem em função da temperatura

a água, a $T=376 K, p=1 \mathrm{~atm}$, a densidade não tem valor fixo, mas há uma escolha entre um valor mais alto, correspondente a densidade do estado líquido, e um valor mais baixo, correspondente ao vapor. Acima do ponto crítico, não há mais escolhas.

Nos exemplos (ii) e (iii) o gráfico que ilustra a relação entre o parâmetro de ordem e a temperatura é uma função decrescente de $T$ que se anula em $T_{c}$. O ponto $T_{c}$ é o ponto final de uma linha de transições de primeira ordem. Em duas dimensões, próximo de $T_{c}$ o comportamento das variáveis (magnetização /densidade) com $T$ é de lei de potência

$$
\begin{gathered}
|M| \approx\left(T_{c}-T\right)^{1 / 8} \\
\rho_{L}-\rho_{G} \approx\left(T_{c}-T\right)^{1 / 3}
\end{gathered}
$$

para $T \leq T_{\mathrm{c}}$.

A existência de um quantidade que é diferente de zero abaixo de uma temperatura crítica, e é zero acima dela é uma característica comum associada aos pontos críticos de grande quantidade de sistema físicos.

Que transições de fase são manifestações da interação entre partículas pode ser bem compreendida pela análise novamente do modelo de Ising, que, como vimos, apresenta transiçào do tipo ordem-desordem. No caso unidimensional, as energias de excitação para levar o sistema de um estado ordenado para um de estados não ordenados são de ordem de grandeza muito menor do que a entropia correspondente, impossibilitando que o sistema se estabilize em qualquer dos estados possíveis. Já em duas e três dimensões, a diferença 
de energia entre o estado ordenado e um estado desordenado qualquer tem a mesma ordem de grandeza da entropia - ambos trese com o volume do sistema -, possibilitando entâa a estabilização do estado ordenado [1]. Como transiçôes de fase são fenômenos de sistemas de volumes infinitos, para considerar todos os infinitos graus de liberdade de tais sistemas no ponto crítico, usamos o procedimento do grupo de renormalizaçăo. Por esse pro cedimento, imaginamos que a rede está dividida em blocos ou células. Se as interaçōes entre os blocos fossem nulas, não haveria correlaçăo a grandes distâncias (maior do que a dimensäo típica de um bloco) entre os blocos. Cono essa são as correlaçōes que produzem divergência nas derivadas de funções termodỉnärnicas, concluínos que as interaçōes importantes para o fenômeno de transição de fase são aquelas entre blocos. 0 método que leva em consideraçẫo tutuaģöes em grandes comprimentos de escala ś conkecido

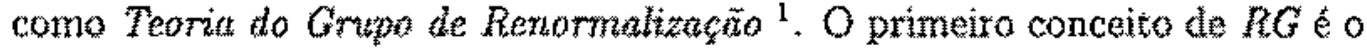
explicado acima de retirar uma fração finita dos graus de liberdade do sistema, efetuando a média sobre eles. O segundo conceito é o de obtençăo de leis de recorrencia com as quais se determina a dependencia do comportamento das grandezas físicas (funçōes de partição) com as escalas dos blocos.

\subsection{Transições de Segunda Ordem}

A questão primeira no estudo de sistemas de spins, em uma rede cúbica com interaçöes quadráticas de curto alcance entre vizinhos tnais próximos, é a de se determinar se tais sistemas têm ordem de longo alcance em baixas temperaturas no limite termodinâmico. Como vimos, estas são as transiç̧ôes de primeira ordem no campo magnético.

Entre os modelos do tipo de Heisenberg (1) os modelos clássicos, isotrópicos e anisotrópicos, ferromagnéticos e antiferromagéticos, em dimensão $\nu \geq 3$ têm transiçăo de primeira ordem [13]; (ii) o modelo quântico isotrópico antiferromagnético também exibe transição de fase em dimensäo $z \geq 3$ para spins $S \geq 1$ [16]; quanto ao modelo quăntico ferromagnético, nada se sabe; (iii) os modelos clássicos, ferromagnéticos e antiferromagnéticos, en $y=2$ dimensöes, têm transiçäo de primeira ordem para todas as anisotropias [I8]; (iv) o modelo quântico antiferromagnético de Heisenberg com arisotropia arbitrária apresenta ordern de longo alcance quando o módulo do valor do

\footnotetext{
IEse netodo foi originalmente elaborado para estudar transiçŏes de segunda ordem
} 
spin, dependente da anisotropia, é suficientemente grande $[17] ;(v)$ o modelo de Ising em $\nu=2$ dimensôes tem estados de equilíbrio com ordem de longo alcance em baixaz temperaturas (o modelo isotrópico de Heisenberg - ferromagnético ou antiferromagnético -, incluindo o rotor, näo tem).

En 1960 Mermin e Wagner [4] provaram que o modelo isotrópico de Heisenberg em duas dimensōes com interaçöes de curto alcance não pode ser ferromagnético nem antiferromagnético, ou seja, nāo há magnetizaçäo espontânea. Stanley e Kaplan [0] apresentaram evidência numérica, baseada em expansöes de altas temperaturas da susceptibilidade, indicando a existencia de uma transiçăo de fase no modelo isotrópico de Heisenberg em duas dimensōes, com interą̧óes ferromagnéticas de visinhos mais prớximos, nâo acompanhadas por ordem de longo alcance. Esse resultado questionava, de um lado, 0 amulamento da magnetizaçāo espontânea - como previsto pelo argumento de ondas de spins - $-e$, de outro lado, a implicaçäo de que a ausencia de ordem de longo alcance seria uma consequência do amulamento da magnetização espontânea.

Parâmetros de orden nunca se anulam abaixo de $T_{6}$, embora possam se anular acima de $T_{5}$. Como o parâmetro de ordem pode tender a zero continuamente quando $T \rightarrow T_{\mathrm{c}}$ por baixo, essa continuidade garante que a transiçäo năo seja de primeira ordem [8]. Notamos ąqui que quando a transiçāo envolve uma quelbra de simetria, o parâmetro de ordem fict bem definido. Por isso podemos tambér dizer que o parămetro de ordem denota uma variável de futuação cujo valor médio é nma medida da ordem ou simetria quebrada no sistema.

\subsection{A Transição de Kosterlitz-Thouless}

Tendo ern vista os conceitos acima, parece já possivel entender a definição formal de transição de Kosterlitz-Thouless, conforme geralmente a encontramos.

A transiçāo de Kosterlitz-Thouless ocorre em uma classe de sistemas bidimensionais, de uma fase de alta temperatura para uma fase de baixa temperatura, caracterizada por o decaimento da correlaçāo obedecer a uma lei de potência. Não hả ordem convencional em ambas as fases, no sentido de que nāo há parâmetro de ordem local, mass a razão de decaimento muda de um 
decaimento polinomial abaixo da temperatura crítica a um decaimento exponencial acima da temperatura critica. Assim, é o comportamento distinto da correlação em alta e baixa temperaturas que distingue as duas hases.

Wegner [9] foi o primeiro a salientar a importăncia da funçăo de correlaçâo como forma de distinguir as duas fases de determinado modelo. Se um modelo sofre transição de fase sem apresentar magnetizaçāo espontânea, é necessấrio encontrar um parâmetro do problema que reconheça suas diferentes fases. A função de correlaçăo funciona como tal parärnetro.

Os sistemas bidimensionais que nos interessam mais de perto quando discutimos a transição de Kosterlitz-Thouless são gases de particulas carregadas. A funçăo de correlaçăo, nesse caso, se relaciona com a interação das cargas do gâs com cargas extermas inseridas no sistema.

Para esse modelos, o fenômeno físico que gera o comportamento distintivo da funçâo de correlaçäo estấ relacionado com uma mudança da forma de agrupamento das cargas no gás. Em certo caso limite do gás, quando a temperatura for suficientemente pequena, todas as partículas estaräo agrupadas aos pares. Em um estreito intervalo de temperatura, a partir de uma temperatura critica, ocorre um processo no qual as cargas, que formavam pares neutros, se desemparelham, e uma transiäo de fase termodinâmica acontece a transiçầ de Kosterlitz-Thouless - a qual tern inicio naquela temperatura crítica do gás. Essa transiçâa foi primeiramente observada por Berezinski, e Kosterlitz e Thouless [11], em sistemas do tipo X-Y nos quais a ordem de longo alcance é destruida por vórtices.

Kosterlitz-Thouless [11] explicaram o mecanismo desse tipo de transiçâo de fase. Eles mostraram que em variáveis angulares, o espaço de configuraçōes pode ser visto como uma superposição de duas configuraçöes independentes $\theta=\theta_{s w}+\theta_{e}$. Correspondenternente, duas contribuiçôes à funçăo de partição tền de ser levadas em conta: uma säo as excitaçôes de ondas de spin que sẫo carnpos sem massa. Apenas essas excitaçôes nâo sāo capazes de gerar uma transiçăo de fase porque estāo relacionadas com a energia cinética, $\mathrm{e}$ sâo responsáveis por destruir a ordem de longo alcance convencional. Outra contribuiçẵo são as excitaçöes de vórtices que interagem como cargas atravês de um potencial bidimensional, e desordenam a função de correlaçăo de spin-spin. A representaçăo 'vórtice-ondas de spin' de sistemas bidimenteionats aparece porque as variatveis na rede são ângulos. A periodicidade nessa variảvel angular dá origem a configurações singulares de spins que destroem a ordem da função de correlação a altas temperaturas. Em trểs dimensões 
a representação anấloga derivada da teoria de gauge na rede é uma representaçāo de 'monopolo-fót ton'; en quatro dimensōes a representaçào é a de 'corrente de monopolo-foton' [14].

\subsection{A Transição em Gases de Coulomb}

Frohlich e Spencer [25] toram capazes de provar a existência ta transição de Kosterlitz-Thouless para vários sistemas bidimensionais na rede, como o gás de Coulomb, o rotor plano, o gás de Villain, o gás de esferas rígidas (hard core), e os modelos $Z_{N},(N \geqslant 1)$. Todos esses sistemas podem ser expressos, por uma tranformaçâa de dualidade, como gases de particulas interagentes através de forças de Coulomb.

Kadanoff [3] fol o primeiro a mostrar que mutitos problemas em Mecânica Estatística podiam ser reeseritos como problemas de gás de Coulomb. Essa associaçāo é possivel, a despeito da natureza de longo alcance do potencial coulombiano, por causa da ocorrencia de blindagem (screening).

Blindagem é um fenômeno da regino de altas temperaturas de sistemas globalmente neutros que causa uma cancelamento do carảcter de longo alcance da força, dexando um potencial efetivo que decai exponencialmente agindo entre grupos de partículas. A força entre a maioria dos pares de partículas distantes é quase inteiramente cancelada pelas forças das partículas restantes na configuraçảo mais provável.

Nesses termos, podemos dizer que a fase de Kosterlitz Thouless è caracterizada pela existência de uma temperatura crítica $\beta_{c}$, tal que, para $\beta<\beta_{c}$, o sistema apresenta blindagem de Debye das cargas externas fracionárias, e, para $\beta>\beta_{\mathfrak{c}}$ nenhuma blindagem ocorre.

Se observarmos o diagrama de fase de estados de equilibrio de um gás de Coulomb - vamos exemplificar com o diagrama do gas de caroço duro $\%$, cujos parâmetros relevantes sẫo a atividade $z$ do gás ê a temperatura inversa $\beta$, notamos que a regita delimitada por $(\beta, z)=(0,0)$ e $(\beta, z)=\left(\infty, e^{\epsilon \beta}\right)$, $\epsilon<1 / 16$, apresenta dois comportamentos diferentes para o gás. Para altas temperaturas e baixas densidades - o dominio de Debye-Huckel - a correlação truncada decai exponencialmente

$$
G_{\beta}^{T}(x, \xi) \leq o(\exp (-m(\beta)|x|) \quad m(\beta)>0
$$


uniformemente no volume para todo $\xi \in(0,1)$. Para essa fase plasmática do gás de Coulomb esse resultado foi provado por Brydges e Federbush [21] e Yang [29].

Quando $\beta$ cresce, uma fase dipolar aparece. Essa é a regiăo da fase de Kosterlitz-Thouless para a qual a correlação $G_{\beta}(x, \xi)$ satisfaz

$$
a(1+|x|)^{-\beta \xi} \leq G_{\beta}(x, \xi) \leq b(1+|x|)^{-c}
$$

para constantes $a_{2} b$ e $c>0$.

Há ainda um terceiro dominio, o de balxas temperaturas para $z>e^{\ell \beta}$, caracterizado pela existểncia de pelo menos dois estados ordenados nos quais as cargas do gás se dispö̌ten em formaçāo cristalina análoga a estrutura do $\mathrm{NaCl}[22$ ].

O gás de Coulomb na rede em duas dimensōes não sofre blindagem se a temperatura e a densidade sāo suficientemente pequenas. Frohlich e Spencer predisseram, por argumentos de entropia-energia, que, para $\beta>8 \pi$, $\alpha$ gás se comportaria cono um gás diluído de dipolos em que grandes dipolos seriam improváveis. A funçăo de correlação entre duas cargas fracionárias opostas teria decaimento polinomial. Esse resultado foi provado por Marchetti e Klein[32] usando o mesmo argumento de energia-entropia numa forma conveniente à prova rigorosa da transição em temperatura inversa $\beta \geq 8 \pi$.

Conforme a temperatura aumenta, deixa de fazer sentido o pensar as cargas como estando ligadas aos pares para a formaçâa de dipolos, e tem-se de tratar com um número igual de partículas livres de sinais opostos que näo sofreram blindagem.

Resultados semelhantes aos citatos acima para sistemas na rede valem para o caso contínuo. Ern particular, para o gás de Coulomb no continuo, citamos a referência [31]. 


\section{Capítulo 2}

\section{Revisão do Gás de Coulomb e Alguns Resultados}

Como mencionamos no primeiro capitulo, gases de Coulomb na rede em duas dimensões exibem a transiça de fase de Kosterlitz-Thouless, a qual ê caracterizada pela existência de uma temperatura crítica, $\beta_{c}$ tal que, para $\beta<\beta_{c}$, a correlação truncada decai exponencialmente, $e$, para $\beta>\beta_{c:}$ a correlação decai polinomialmente.

Neste capítulo fazemos uma revisäo de sistemas coulombianos que exibern a transição de fase de Kosterlitz-Thouless. Damos definiçöes e citamos alguns resultados para funçôes de correlação. O resultado principal - prova de limite inferior para o operador de desordem - aparece no Capítulo 4 para o gás de Villain. A razão da escolha dessse gás é que nosso sistema quântico de rotores será mapeado em urn gás de Villain, para o qual a prova deve valer, ainda no caso em que o espaçamento de rede em una direção seja contínuo. $O$ limite inferior para a funç̧ão de correlaçăo - que, juntamente com a stimativa para o operador de desordem mencionada acima, completa a prova da transiçäo do Kosterlitz-Thouless para o gás de Villain - será derivado no Capstulo $\theta$ para o gás obtido do mapeamento do rotores.

\subsection{Gases de Coulomb}

Consideramos um sistema de partículas clássicas com cargas elétricas inteiras interagindo através de urn potencial coulombiano de cargas estáticas em 
alguma região finita $\Lambda \subset Z^{2}$. A cada sítio $x \in \Lambda$ associamos uma carga elétrica total $q(x)$ concentrada em $x$.

Uma configuração do sistema é dada pela função

$$
q_{\Lambda}: \Lambda \rightarrow Z, x \in \Lambda \rightarrow q(x) \in Z
$$

A energia eletrostática de uma configuração $q_{\Lambda}$ é

$$
\begin{aligned}
E\left(q_{\Lambda}\right) & =\frac{1}{2} \sum_{x, y \in \Lambda} q(x) q(y) C(x, y) \\
& \equiv \frac{1}{2}\left(q_{\Lambda},(-\Delta)^{-1} q_{\Lambda}\right),
\end{aligned}
$$

$C(x, y)$ é a funçāo de Green do laplaciano de diferença finita $\Delta$ com condições de contorno de Dirichilet em $\Lambda$.

Vamos nos restringir a configuraçōes neutras únicas cujas energias permanecem finitas quando $\Lambda \uparrow \mathbf{Z}^{2}$.

$O$ fator de Boltzmann de uma configuração $q_{\Lambda}$ é

$$
e^{-\beta E\left(q_{\Lambda}\right)}, \quad \beta=1 / T
$$

e o peso a priori

$$
\prod_{x \in \Lambda} \lambda(q(x))
$$

cujas medidas satisfazem, para cada $q \in Z$, as propriedade de uma distribuição chamada típica:

a) $\lambda(q)=\lambda(-q)$

b) $|\lambda(q)| \leq e^{v q^{2}}, \quad$ para algum $v$.

$\mathrm{O}$ estado de equilíbrio do sistema é definido pela medida de Gibbs

$$
d \mu_{\Lambda}\left(q_{\Lambda}\right)=Z_{\Lambda}^{-1} e^{-\beta E\left(q_{\Lambda}\right)} \prod_{x \in \Lambda} \lambda(q(x))
$$

onde

$$
\mathcal{Z}_{\Lambda}=\sum_{q_{\Lambda}=\{q(x)\}_{x} \in \Lambda} e^{-\frac{g}{2}\left(q_{\Lambda},(-\Delta)^{-1} q_{\Lambda}\right)} \prod_{x \in \Lambda} \lambda(q(x)) .
$$

é a função de partição .

Em duas dimensões a função de Green é dada por

$$
C(x, y)=\frac{1}{(2 \pi)^{2}} \int_{-\pi}^{\pi} d k_{1} \int_{-\pi}^{\pi} d k_{2} \frac{e^{2 \vec{k} \cdot \vec{x}}}{4-2 \cos k_{1}-2 \cos k_{2}}
$$


Note que esca função é sempre positiva, mas nẫo é finita em $\left(k_{1}, k_{2}\right)=(0,0)$. No entanto, impondo a condiçẫo de neutralidade ${ }^{1}$ a função de Green fica bem definida em todo seu domínio. Isso pode ser visto da seguinte forma: decompomos a funçăo de Green em duas partes

$$
C(x, y)=C^{\prime}(x, y)+C(0,0)
$$

onde $C^{t}$ e finita no limite infravermelho

$$
C^{\prime}(x, y)=\frac{1}{(2 \pi)^{2}} \int_{-\pi}^{\pi} d k_{2} \int_{-\pi}^{\pi} d k_{2} \frac{\mathrm{e}^{2 \vec{k} \cdot \vec{x}}-1}{4-2 \cos k_{1}-2 \cos k_{2}}
$$

e

$$
C(0,0)=\frac{1}{(2 \pi)^{2}} \int_{-\pi}^{\pi} d k_{1} \int_{-\pi}^{\pi} d h_{2} \frac{1}{4-2 \cos k_{1}-2 \cos k_{2}}
$$

Substituindo as expressőes acima na funçäo de partiçäo obtemos explicitamente

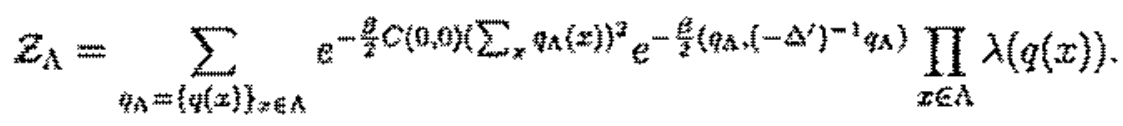

onde $A^{\prime}$ o o laplaciano para o qual a funçăo de Green correspondente é $C^{\prime}$. Dessa forma vemos que a exponencial seleciona automaticamente as configuraçoes neutras $\left(\sum_{x} g_{n}(x)=0\right)$.

Os seguntes modelos especiais [27] podem ser tratados simultaneamente:

1) O găs de esferas rugidas

No gás de esferas rigidas, uma tinica particula, independentemente de sua carga, pode ocupar um sitio da rede, de modo que a repulsäo entre duas partículas posicionadas no sisterna é infinita. A cada partícula da rede esta associada uma atividade $z$, independentemente da carga. A funçào de distribuição das cargas a prion è dada por

$$
d \lambda(q)=\delta_{q, 0}+z\left(\delta_{q, 1}+\delta_{q,-1}\right)
$$

onde $\delta$ é a funçäo de Dirac.

2) O güs standard

\footnotetext{
${ }^{i}$ Densidade média de carga 6 zero
} 
O gás standard é aquele en que qualquer número de particulas pode ser inserido em cada ponto da rede. Cada partícula, independentemente de sua carga, tem associada a si uma atividade $z$ e uma carga a. A funçầo te distribuiçăo das cargas a pronto dada por

$$
\lambda(q)=\frac{I_{n}(2 z)}{I_{0}(2 z)} \text { para } q=0, \pm 1, \pm 2, \ldots,
$$

onde $z$ é a atividade que controla a densidade do sistema, $I_{q}$ denota a funçäo de Bessel modificada de ordem $q_{y}$ ou seja, o coeficiente $q$ da transformada de Fourier de $\exp z \cos \phi$;

3) O gús de Villain

$$
\lambda(q)=1 \text { para } q=0, \pm 1, \pm 2, \ldots
$$

\subsection{A Representação de sine-Gordon do Gás de Coulomb}

Na representação de sine-Cordon (ou Siegert), a função de partiçäo do sistema coulombiano é escrita como uma mêdia sobre um funcional de funçôes aleatórias gaussianas. A média é o funcional gerador de uma teoria de sineGordon. Essa representação é especialmente conveniente para descrever as propriedades do estado de equilibrio como, por exemplo, a blindagern de Debye.

Seja $\phi: Z^{\nu} \rightarrow R$ o campo gaussiano em $Z^{\nu}$ com distribuição

$$
d \mu_{\beta}(\phi)=N^{-1} \prod_{j} d \phi(j) \exp \left[\frac{1}{2 \beta}(\phi, \Delta \phi)\right]
$$

onde

$$
(\phi, \Delta \phi)=-\sum_{|i-j|=\mid}(\phi(i)-\phi(j))^{2}
$$

e

$$
N=\operatorname{det}\left(-\frac{\Delta}{2 \pi \beta}\right)^{-1 / 2}
$$

co fator de renormalizagâa .

O funcional característico da medida gaussiana com média 0 e covariància 
$\beta(-\Delta)^{-1}$ é dado por

$$
\int e^{x(\phi, \rho)} d \mu_{\beta}(\phi)=e^{-\frac{\beta}{2}\left(\rho,(-\Delta)^{-1} \rho\right)}
$$

A densidade $\rho(x)$ é a variável conjugada ao campo escalar $\phi$ em $R^{2}$ - é uma fonte - , e $\phi(\rho)=\sum \phi(x) \rho(x)$, com $\rho(x)=\sum_{j} q_{j} \delta\left(x-x_{j}\right)$.

A substituiçāo da expressāo (2.2) acima na função de partição fornece

$$
\mathcal{Z}=\int d \mu_{\beta}(\phi) \sum_{q_{\Lambda}} e^{\imath \phi(q)} \prod_{x \in \Lambda} d \lambda(q(x))
$$

Perfazendo a transformada de Fourier da medida de Gibbs com respeito às variáveis de carga $\{q(x)\}$ obtemos uma representação equivalente do gás de Coulomb como uma sine-Gordon da teoria quântica euclidiana de campos

$$
Z_{\Lambda}=\int \prod_{x, t} \hat{\lambda}(\phi(x, t)) d \mu_{\beta}(\phi)
$$

onde

$$
\hat{\lambda}(\phi(x))=\sum_{q_{\Lambda}} e^{2 \phi(q)} \prod_{x \in \Lambda} d \lambda(q(x))
$$

1) Pata o gás de esferas rigidas

$$
\hat{\lambda}_{z}(\phi)=1+z \cos \phi
$$

2) Para o gás standard

$$
\hat{\lambda}_{z}(\phi)=e^{z \cos \phi}
$$

3) Para o gás de Villain (com $\zeta: 1$ em (2.1))

$$
\hat{\lambda}_{z}(\phi)=\sum_{n} \delta(2 \pi n-\phi)
$$

onde usamos a fórmula de soma de Poisson:

$$
\sum_{n=-\infty}^{\infty} \delta(\phi-n)=\sum_{k=-\infty}^{\infty} e^{-\imath 2 \pi k \phi}
$$

para obter a transformada de Fourier da unidade. 


\subsection{A Função de Correlação}

A função

$$
\left\langle e^{\imath \phi(f)}\right\rangle_{\Lambda}(\beta, \lambda)=Z_{\Lambda}^{-1} \int e^{-\beta E\left(q_{\Lambda}+f\right)} \prod_{x \in \Lambda} d \lambda(q(x))
$$

mede a correlaçāo entre cargas externas $f(x)$, localizadas em sítios diferentes de $\Lambda$, que foram inseridas de fora no sistema. Assim, consideramos a distribuição de equilíbrio para uma situação em que uma carga fracionária $\xi$ está fixada na origem, e verificamos o que ocorre quando outra carga de intensidade oposta àquela é inserida no sistema no ponto $x>0$. A situação é descrita pela funçāo de correlaçāo de cargas externas, análoga à dada acima:

$$
G_{\Lambda}^{\xi}(x)=\frac{Z_{\Lambda}^{\xi}(x)}{Z_{\Lambda}}
$$

onde

$$
Z_{\Lambda}^{\xi}(x)=\int e^{-E\left(q_{\Lambda}+\xi\left(\delta_{0}-\delta_{x}\right)\right)} \prod_{j \in \Lambda} d \lambda(q(j))
$$

e $f(j)=\xi\left(\delta_{j 0}-\delta_{j x}\right)$, onde $\xi: \Lambda \rightarrow \mathbf{R}$ é a distribuição de cargas externas.

Como se sabe, o comportamento da função de correlação reflete as propriedades de blindagem do gás de Coulomb.

A funçāo de correlação também pode ser escrita na representação de sineGordon como

$$
G_{\Lambda}^{\xi}(x)=Z_{\Lambda}^{-1} \int d \mu_{\beta}(\phi) e^{t(\xi, \phi)} \prod_{x \in \Lambda} \hat{\lambda}(\phi(x))
$$

Resultado 2.3.1 (Frohlich-Spencer) Para o gás de Coulomb de esferas rígidas,

$$
G^{\xi}(0, x)<\text { const. }(1+|x|)^{-\delta(\beta, \xi)}
$$

para algum $\delta(\beta, \xi)>0$, para todo $\beta>\beta(\xi, z)$.

\subsection{Limite Inferior da Função de Correlação}

O primeiro passo para provar a existência da transiçāo de Kosterlitz-Thouless consiste em encontrar um limite inferior para a funçāo de correlaçāo . Isso é feito por meio da desigualdade de Jensen aplicada à variável de carga. Como esse resultado é de mesma natureza daquele demonstrado para os rotores 
quânticos no Capitulo 6, aqui apenas o citamos sem provas.

Temos que

$$
G_{7}(x) \geq|x|^{-3 / 2 \pi}
$$

para $z \leq e^{\epsilon \beta}, \beta>\beta_{c}$ para algum $\epsilon>0$.

Se blindagem de Debye ocorre,

$$
G_{\lambda}^{\varepsilon}(x) \rightarrow C>0
$$

exponencialmente rápido quando $|x| \rightarrow \infty$.

\subsection{A Função de Partição de Cargas Externas}

Na representaçäo de sine-Gordon a funçäo de partição na presença de cargas externas fica

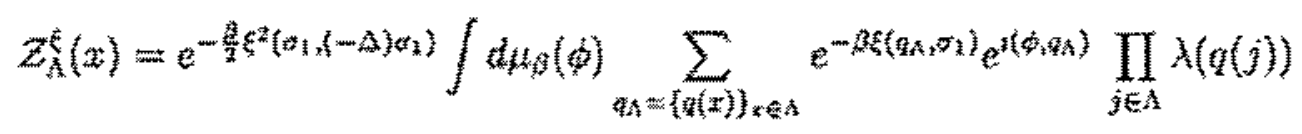

onde introduzimos

$$
a_{1}(t)=(-\Delta)^{-1}\left(\partial_{1} f^{x}\right)(j)
$$

e a funçäo $f^{x}$ te definida por

$$
f^{x}\left(j_{1}, j_{2}\right)= \begin{cases}1, & \text { if } 1 \leq j \leq x, j_{2}=0 \\ 0, & \text { otherwise. }\end{cases}
$$

onde $\partial_{\alpha} \alpha=1,2$ e a derivada de diferença finita na direçäo $\alpha$, definida por

$$
\left(\partial_{\alpha} f\right)(j)=f\left(j+e_{\alpha}\right)-f(j)
$$

onde $e_{\alpha}(\alpha=1,2)$ é o vetor unitário na direção $\alpha$. Alternativamente

$$
Z_{\lambda}^{\xi}(x)=e^{-\frac{\beta}{2} \xi^{2}\left(\alpha_{x},\{-\Lambda) \sigma_{1}\right)} \int d \mu_{\beta}(\phi) \hat{\lambda}_{x}(\phi)
$$

mas agora

$$
\hat{\lambda}_{x}(\phi)=\sum_{q} e^{q \phi q+\beta \xi_{\sigma_{1} q}} \lambda(q)
$$




\subsection{O Operador de Desordem}

Estabelecer um limite inferior para o valor esperado to operador de desordem [8] pode ser usado en substibição a caleular um limite superior - este más difícil - para a função de corretução. Na representação do gas chamamos operador de desordem ao operador

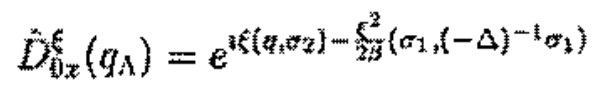

onde

$$
\sigma_{2}(j)=(-\Delta)^{-1}\left(\partial_{2} f\right)(j)
$$

para $\leqslant \leq(0,1)$.

$O$ valor esperado é entro

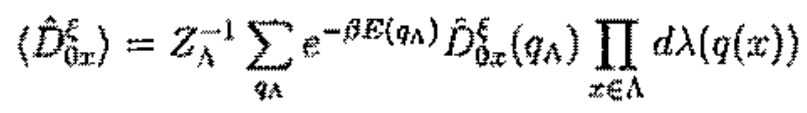

Usando (2.2) podemos escrever, na representação de šne-Gordon,

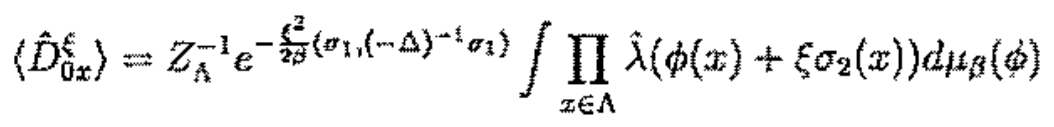

Repare que

$$
G(x)=e^{-\frac{x^{*}}{37}\left(\sigma_{1}(-\Delta)^{-1} \sigma_{1}\right)}
$$

6 a contribuição las ondas de spin ao valor esperado do operador de desordem.

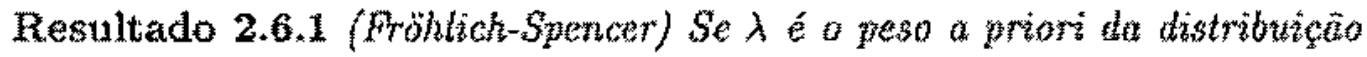
de um gás te Coulomb geral satisfazende a condiço de crescimento

$$
|\lambda(q)| \leq \text { consi. } e^{(\alpha+<b) q^{\prime \prime}}, \quad \varepsilon<1 / 16
$$

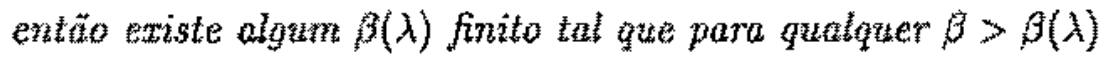

$$
(\hat{D})(\rho, \lambda) \geq \text { const. } e^{-\frac{f^{z}}{2 \beta^{T}} \ln (1+|x|)}
$$

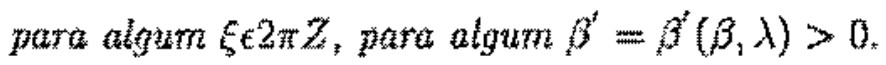


Resultado 2.6.2 (Frohlach-Spencer) Para o modalo de Villon, para coda $0<\xi<1$ existe algum $\beta_{0}(\xi)$ finato sal mue para $\beta>p_{0}(\xi)$

$$
\left(D_{0}\right)(\beta)<\text { const }(1+|x|)^{-\xi^{z} p^{t}}
$$

para algum positizo $\beta^{t}=\beta^{*}(\rho, \xi) \rightarrow \infty$ quando $\theta \rightarrow \infty$. Por expansöes de altas temperaturas

$$
\left(D_{0 x}^{*}\right)(j) \geq \text { const. }>0
$$

unitomenente em $[x]$ quando $\rho$ é suficientemente pequeno. 


\section{Capítulo 3}

\section{Estimativas de Correlação na Representação das Cargas}

\subsection{Introdução}

Antes de prosseguir com os gases de Coulomb fazemos uma pausa para discutir a obtenção de estimativas de correlação em gases de dipolos. Há alguns motivos para isso. Em primeiro lugar, é interessante observar que apesar da interpretação correta fornecida por Kosterlitz e Thouless em 1973 da existência de urna transição de fase no estado fundamental do rotor planoou o modelo $X Y$-, até 1981 nenhuma prova matemática havia dessa transiçäo.

A técnica de prova da transição de Kosterlitz-Thouless, que visa a obter os comportamentos da funçäo de correlaçäo deseritos no capítulo anterior, foi primeiramente desenvolvida e aplieada a gases de dipolos por Frohlich e Spencer [24].

Embora os próprios gases de dipolos não apresentem a transição de Kosterlitz-Thouless, des constituem um ponto de partida natural para o estudo dessa transiģăo em gases de Coulomb porque cargas em täis gases tendem a formar pares com cargas opostas, à semelhança de dipolos, em baixas temperaturas e petuenas atividades.

Aprimorada para poder ser aplicada a gases de Coulomb em geral, essa técnica permitiu a prova da existência da transiçâo de Kosterilizz-Thouless para o gás de caroço duro, o gás de Villain, os rotores, os modelos $\mathbf{Z}_{N}$. 
O principal ingrediente da tếcrica consiste na aplicação da transformaşão de sine-Gordon, Eq. (2.2), tu funçẫo de partiçāo dos gases. É isso o que faremos no prớximo capítulo para o gấs de Villain.

Neste capítulo, porểm, dispensamos a trarşormaçâo de sine-Gordon, e trabalhamos diretamente na representação das cargas do gás de dipolos.

Cono se sabe, diferenternente do comportamento da correlaçāo em sistemas coulombianos, correlaçōes de carga em gases de dipolos tem limite inferior e superior que decaem polinomialmente. O limite inferior é consequência da desigualdade de Jensen aplicada às vartáveis de carga. Jả vimos no Capítulo 2 a que resultados essa desigualdade leva para a correlação de gases de Coulomb. Voltaremos a aplicá-la aos rotores quânticos no Capítulo 6, depois de mapeá-los em um sistema clássico bidimensional.

$E$ É da obtençäo do limite superior para a função de correlação que tratamos aqui. Esperamos que os pormenores da prova que daremos no próximo capitulo para o gás de Villain possam ser mais facilmẹțe entendidos a partir da análise do gás de dipolos.

É um resultado bem conhecido da teoria de campo médio que a correlaçăo dipolar é proporcional à energia de interaçâo de un par de dipolos, com fator de proporcionalidade dado pelo inverso da constante dielétrica $d(z, \beta)$. Esta, por sua vez, é dada pela série

$$
d=1+\sum_{m+n>0} a_{m a n} z^{n} p^{m},
$$

onde $z$ a atividade, e $\beta$ a temperatura inversa.

Os coeficientes $a_{m n}$ săo derivados por tooria de perturbação. Nosso resultado fornece $o$ termo de primeira ordern em $2 \beta$, com $a_{11}=(1+\epsilon)$, para qualquer $\epsilon>0$.

Primeiramente consideramos o mais simples gás de dipolos de comprimento fixo, com orientaçầ discreta, que năo se sobrepôem. Em seguida generalizamos para dipolos de dois comprimentos diferentes. Vamos sempre supor que as cargas externas interagentes com o gás estaräo bem afastadas da regiäo onde os dipolos se encontram. Tais simplificą̧oes visam a reduzir o método de obtençâo de estimativas para a correlação ao essencial da transiação de cargas, e enfatizar a utilizaçäo direta da expansảo de cluster na representação das cargas.

Deixaremos para o próximo capitulo, o problema completo da transição de Kosterlitz-Thouless em gases de Coulonb, mas lá estaremos de volta à re- 
presentaçăo de sine-Gordon.

\subsection{A Função de Partição de Gases de Dipo- los}

Um dipolo de rede é um par de cargas elétricas de mesma intensidade mas sinais opostos situadas em sítios vizinhos da rede.

Consideramos um gấs de dipolos com orientação disereta em uma regiäo quadrada finita $\Lambda$. Seja $l=4 r Z^{2}$ uma sub-rede de $A$ de espaçamento $4 r$, contida en uma caixa $\hat{\Lambda}$ dentro de $\Lambda$ e concêntricu com ela.

Uma configuração dos dipolos é descrita pela função $\vec{\mu}_{L}=\{\vec{\mu}(j)\}$, ondes $\vec{\mu}(j)=\left(\mu_{1}(j), \mu_{2}(j)\right)$ e momento de dipolo total no sitio $j \in L$ da rede.

A funçăo de partiçăo é dada por

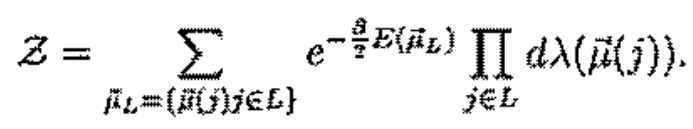

onde

$$
E\left(\vec{\mu}_{L}\right)=\sum_{j, k} \sum_{\alpha, t} \hat{m}_{\alpha}(j) \mu_{\gamma}(k) W_{\alpha \gamma}(j, k)
$$

O potencial dipolar é dádo por

$$
W_{\alpha \gamma}(j, k)=\partial_{\alpha} \partial_{g} C(j, k)
$$

onde $\partial_{\alpha}$ foi definida em $(2.5)$.

Vamos nos restringir à classe de gases de dipolos coulombianos com caroço duro para o qual $C(j, k)$ é a função de Green de um potencial de Coulomb na rede bidimensional, dado pela solução fundamental da equação de Laplace na rede:

$$
C(j)=(-\Delta)^{-1}(j)=\frac{1}{(2 \pi)^{2}} \int_{-\pi}^{\pi} d^{2} \zeta \frac{e^{1 \zeta \cdot j}}{2 \sum_{\alpha}\left(1-\cos \zeta_{\alpha}\right)}
$$

O peso a prioni da distribuiçāo é dado por

$$
\lambda(\vec{\mu})=\delta(\vec{\mu})+z \delta\left(|\vec{\mu}|^{2}-1\right)
$$


onde $z$ é a atividade ou fugacidade.

Nă haverá sobreposição de dipolos se seu comprimento for fixo e igual a $\%$ Por simplicidade tomaremos $r=1$.

A comrelaça de duas cargas externas colocadas no sistema, uma em $+\xi$, situada em $t$, outra $-\xi$ em $w_{1}$ dada por $(2.3)$

$$
G(x, y)=\frac{Z_{L}^{E}(x, y)}{Z_{L}}
$$

onde a função de partição na presença dessas cargas extemunt

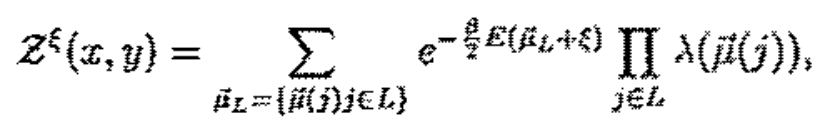

$\operatorname{com} \xi=\xi(\delta(j-x)-\delta(j-y))$

Vamos sempre supor que $w$ e $y$ näo pertencam a $A$.

\subsection{A Desigualdade Eletrostática}

O primeiro passo para encontrar un limite superior para a funçăo de correlação consiste em reprimir a atividade dos dipolos, de modo a obter estimativas válidas para valores pequenos de $z$. Isso é feito pela desigualdade eletrostática (Apêndice $A$ ). Para aplicálạ, é mais conveniente efetuar a soma sobre os momentos de dipolo em (3.1), o que dá

$$
\mathcal{Z}=\sum_{\left\{\mu_{1}(j), \mu_{2}(j), j \in L_{j}\right\}} e^{-\frac{g_{2}}{2} E\left(\vec{\mu}_{\prime}\right)} \prod_{j, \alpha} z_{\alpha}(j)
$$

onde $z_{a}(j)=z(j)^{\left|p_{a}(j)\right|}$.

Se definimos a densidade de carga do gás de dipolos por

$$
p(j)=\sum_{a} b_{\alpha} H_{\mathrm{Fx}}(j)
$$

onde $e_{\alpha}(\alpha=1,2)$ é o vetor unitário de rede na direçăo $\alpha$, a cutergía eletrostatitua pode ser escrita como

$$
E\left(\vec{H}_{L}\right)=E(\rho)=\left(\mu, \mathrm{s}^{-1} \mu\right)
$$


Substituimos a dersidade de carga $p$, cujo suporte esta em $\hat{\Lambda}$, por uma densidade de carga $\vec{\beta}$, cujo suporte situa-se em $A$, de tal modo que a energia de interaçäo entre as tuas densidades de carga renormalizadas näo se altere, mas a auto-energia de $\ddot{p}$ seja menor do que a auto-energia de $p$. Usamos a diferença entre as auto-energias para renormalizar as atividades.

A densidade de carga renormalizada é dada por

$$
\bar{\rho}=\rho+\frac{\Delta \rho}{\|\Delta\|}
$$

com o propósito de renormalizar a atividade tos dipolos, escrevemos [22]

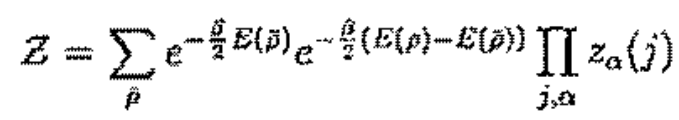

A diferença em auto energia é calculada como segue:

$$
\begin{aligned}
E(\rho)-E(\bar{\rho}) & =\frac{2}{\|\Delta\|}(\rho, p)-\frac{1}{\|\Delta\|^{2}}(\rho,-\Delta \rho) \\
& \geq \frac{1}{\|\Delta\|}(\rho, p)
\end{aligned}
$$

Obtemos

$$
Z=\sum_{\tilde{\rho}} e^{\infty \frac{\beta}{2}\left(\ddot{p}_{1}-\Delta^{-1} \ddot{\rho}\right)} \prod_{j, \alpha} \vec{z}_{\alpha}(j)
$$

com a estimativa

$$
z_{\alpha}(j) \leq z_{\alpha}(j) \exp \left\{-\frac{p}{2\|\Delta\|} p^{2}(j)\right\}
$$

Como as atividades tëm todas o mesmo valor, as atividades renormalizadas podem ser limitadas por (fazendo $\|\Delta\|=8$ )

$$
\bar{z}_{\alpha}(j) \leq z=z \exp \left\{-\frac{\beta}{16}\right\}
$$

a qual pode ser feita arbitrariamente pequena para $\beta$ suficientemente grande.

\subsection{As Cargas Externas}

Aplicamos o raciocínio acima à função de partição na presença de cargas externas, e obtemos

$$
\mathcal{Z}^{\xi}(x, y)=\sum_{\vec{p}} e^{-\frac{\bar{a}}{2} E(\dot{p}+\xi)} \prod_{j, \alpha} \bar{z}_{\alpha}(\hat{j})
$$


Nesse caso, no entanto, precisaremos renomalizar as cargas externas também. Isso ç feito pela tranciàçāo

$$
\bar{\xi}=\xi+\frac{\eta}{\beta}(\Delta a)(j)
$$

onde $\gamma$ depende de $\beta$ de um modo ainda a set determinado,

$$
a(j)=C(j, x)-C(j, y)
$$

A unçâa $a(j)$ satisfaz, para $|x-y|$ grande e para $\left|y-j^{2}\right|=1$, o seguinte

$$
\begin{gathered}
\left|a(j)-a\left(j^{\prime}\right)\right| \leq \text { const } \mid \frac{1}{|j-x+1|}+\frac{1}{|j-y+1|} \\
|a(j)-a(j)| \leq \text { const } \frac{|x-y|}{|j|^{2}}
\end{gathered}
$$

Essas extimativas podem ser deduzidas das propriedades correspondentes da funçāo de Green para $|x-y|$ grande, como dado em [20].

Da defintegto do operador laplaciano e (3.12), temos também que

$$
\sum_{\left|j-j^{\prime}\right|=1}\left[a(j)-a\left(j^{3}\right)\right]^{2} \leq(a,-\Delta a)=a(x)-a(y)
$$

Para $|x-y|$ grande, o operador de covariancia é bem atproximado pelo prom pagador contínuo sen massa em duas dimensōes [2]. Assim

$$
\begin{aligned}
a(x)-a(y) & =2[O(0,0)-C(x, y)] \\
& \approx \frac{1}{\pi} \log |x-y| .
\end{aligned}
$$

Depois da translatâno obtemos, in mesma maneira que em (3.8),

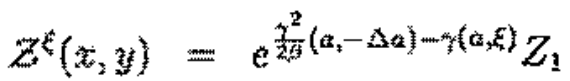

$$
\begin{aligned}
& \left.=E^{\left(\frac{n^{2}}{2}\right.}-\gamma\right)\left(a_{1}-\Delta a\right) Z_{1}
\end{aligned}
$$

onde usamos que $a(j)=\left[\left(-\mathrm{A}^{\cdots}\right) \xi\right](j)$ de modo que $(a, \xi)=\left(a_{3}, \mathrm{~B} a\right)$, e

$$
Z_{1}=\sum_{\bar{p}} e^{-\frac{3}{2} E(\bar{\rho}+\bar{\xi})} \prod_{j, a} z_{t a}(j) e^{\gamma_{\alpha}(j)}
$$

com

$$
\gamma_{\alpha}(j)=\gamma \mu_{\alpha}(j) \delta_{\alpha} a(j)
$$

e, como en (3.7).

$$
\bar{a}(j)=a(j)+\frac{\Delta}{\|\Delta\|} a(j)
$$




\subsection{A Expansão de Cluster}

Até açui, vimos aplicando o mêtodo de translações reais para renormalizar as atividades de carga. Esse método é equivalente ao método das translaçöes complexas introduzido em [24] usando a representação de sine-Gordon.

Agora precisamos de um novo procedimento para derivar um limite superior para a funçăo de correlaçăo. Comeşamos por expandir $\prod_{j, a} \exp \left\{\gamma_{\alpha}(j)\right\} \mathrm{em}$ cluster $[28]$

$$
\prod_{j, a}\left[1+\left(e^{\gamma_{a}(j)}-1\right)\right]=\prod_{a}\left(1+S_{\alpha}+P_{\alpha}\right)
$$

onde

$$
S_{\alpha}=\sum_{m_{\alpha} \geq 1} \sum_{j_{1}, \ldots, j_{m_{\alpha}}} D_{\alpha}\left(j_{1}\right) \ldots D_{\alpha}\left(j_{m_{\alpha}}\right)
$$

com

$$
D_{\alpha}\left(j_{i}\right)=\cosh \gamma_{a}\left(j_{i}\right)-1
$$

A soma (3.21) corre sobre todas as subsequências de $\left\{j_{1}, \ldots j_{m_{\alpha}}\right\}, m_{\alpha} \geq 1$. Temos também que

$$
P_{\alpha}=\sum_{\tau_{\alpha}}^{\prime} \prod_{j}\left(\cosh \gamma_{\alpha}(j)\right)^{\tau_{\alpha}(j)}\left(\sinh \gamma_{\alpha}(j)\right)^{1 \cdots \tau_{\alpha}(j)}
$$

onde $\tau_{\mathrm{i} \alpha}=\left\{\tau_{\alpha}(j)=0,1\right.$ para todo $\left.j\right\}$, e a soma é efetuada sobre todos os conjuntos de valores de $\tau_{a x}$ não identicamente 1 , visto que esse termo já aparece en $S_{\alpha}$.

Fazendo a raultiplicação sobre $\alpha$ en (3.20) e substituindo o resultado em $Z_{1}$, obtemos

$$
Z_{1}=\sum_{\tilde{z}} e^{-\frac{a}{z} E(\hat{a})} \prod_{j, \alpha} \tilde{z}_{\mathrm{w}}(j)\left(1+S+P+S_{1} P_{2}+S_{2} P_{1}\right)
$$

onde $S=S_{1}+S_{2}+S_{1} S_{2}$ eP= $P_{1}+P_{2}+P_{1} P_{2}$.

Nesse ponto convem levar a cabo explicitamente o cálculo dos termos envolvidos ma expansäo de cluster. Nāo é dificil ver que a soma de termos em (3.24) fornece

$$
Z_{1}=Z+\sum_{\bar{x}} e^{-\frac{B}{2} E(\hat{j})} \prod_{j, \alpha} \bar{z}_{\alpha}(j)\left(S_{1}+S_{2}+S_{1} S_{2}+R\right)
$$

\footnotetext{
'Seremos demasiatamentat gerais ą̧ui. Isso se justifica, no entanto, porque quteremos desenvolvar um mettoto, a tamberm porque temos em mente a aplicaçäo da expansăo de clustor em situatoses mals complexas do cue simples gases de dipolos.
} 
onde $\mathcal{Z}$ é a função de partiçäo dada em (3.9), e

$$
R=\prod_{j, \alpha}\left(\cosh \gamma_{\alpha}(j)+\sinh \gamma_{\alpha}(j)\right)-\prod_{j, \alpha}\left(\cosh \gamma_{\alpha}(j)\right)
$$

\subsection{O Limite Superior}

Temos a estimativa bbvia entào

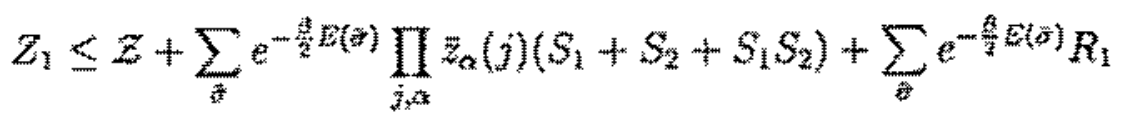

onde

$$
R_{1}=\prod_{j, \alpha}\left(\ddot{z}_{\alpha}(j) \cosh \gamma_{\alpha}(y)+z_{\alpha}(0) \sinh \gamma_{\alpha}(0)\right)
$$

A funçằ $\gamma_{\alpha}(j)$ depende de $\delta_{\alpha} a(j)$. Todavia, note que

$$
b_{a z}(j)=(a(y)-a(j+e)=a(j)-a(j+e)
$$

quando $j ; x_{x} y$, pois

$$
\ddot{a}(j)-a(j)=\frac{1}{\|\Delta\|}(\Delta a)(j)=0
$$

para $j \neq x, y$.

De acordo com a definiçấo (3.19) de $\gamma_{\alpha}$ e as estimativas (3.13), (3.14) para $\left|a(j)-a\left(j^{\prime}\right)\right|$, o argumento de cosh $(\cdot)$ é pequeno para $|j-x| \gg \gamma$ and $|j-y| \gg$ $\gamma$. Então podemos escrever

$$
\cosh \gamma_{\alpha}(j)-1 \leq \frac{1}{2}(1+\epsilon) \gamma^{2}[a(j)-a(j)]^{2}
$$

para algum $\epsilon>0$.

Para $|j-x| \leq O(\gamma),|j-u| \leq O(\gamma)$, de novo por (3.19) e (3.13),(3.14), estimamos $\cosh (\cdot)-1$ por uma constante dependente de $\gamma$.

Em ambos os casos, cada fator em (3.26) pode ser limitado pela unidade para $z$ suficientemente pequeno, e consequentemente, $\left|R_{1}\right| \leq 1$. Além dísso, podemos sempre fazer $\beta$ täo grande que $\sum_{\tilde{\sigma}} \exp -\frac{\beta}{2} E(\bar{\sigma})<1$. Nessas circunstâncias, podemos eliminar o último termo em $(3.25)$ :

$$
Z_{1} \leq K_{\beta}\left\{z+\sum_{j} e^{-\frac{g}{2} E(\bar{\partial})} \prod_{j, a} z_{\alpha}(j)\left(S_{1}+S_{2}+S_{1} S_{2}\right)\right\}
$$


para alguma constante $K_{\hat{p}}$.

Podemos distribuir as atividades nos produtos de $S_{\alpha}$. Isso dá

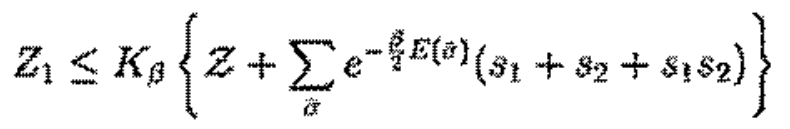

onde $s=s_{1}+s_{2}+s_{1} s_{2}$ e, como em (3.21),

$$
s_{\alpha}=\sum_{m_{\alpha} \geq 1} \sum_{j_{1}, \ldots, j_{m_{\alpha}}} z_{\alpha}\left(j_{1}\right) D_{\alpha}\left(j_{1}\right) \ldots \bar{z}_{\alpha}\left(j_{m_{\alpha}}\right) D_{\alpha}\left(j_{m_{\alpha}}\right)
$$

Vemos entäo que $Z_{1}$ admite o limite superior

$$
Z_{1} \leq K_{1} Z\left(1+s_{1}+s_{2}+s_{1} s_{2}\right)
$$

Aqui os termos $s$ tomam seus valores máximos, os quais ocorrem quando $\mu_{\alpha}(j)=1$ para todo $j_{2} \alpha$, e usamos que $Z>1$.

A equaçäo (3.32) tem o mesmo padräo da equaçăo (3.20) expandida exceto que aqui inserimos as atividades em $S$. Se re-somamos a expansāo de ciuster em sua forma original, vem

$$
Z_{1} \leq K_{\beta} z \prod_{j, \alpha}\left(1+z_{\alpha}(j) D_{\alpha}(j)\right)
$$

com $\mu_{\alpha}(j)=1$ para todo $j, \alpha$.

Substituindo (3.33) em (3.17) e usando (3.11) e (3.15), obtemos

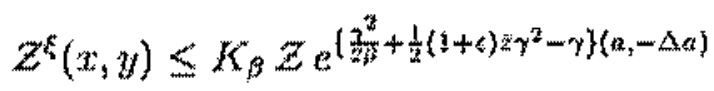

Finalmente, derivando o coeficiente

$$
\frac{\gamma^{2}}{2 \beta}+\frac{1}{2}(1+\epsilon) z \gamma^{2}-\gamma
$$

com relaçäo a $\gamma$ obtemos a escolha btima de $\gamma$

$$
\gamma=\beta(1+(1+c) z \beta))^{-1}
$$

a qual fornece, usando $(3.14)$ e (3.15),

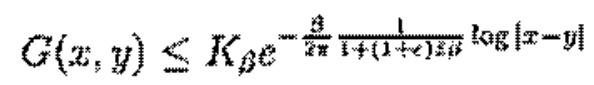




\subsection{Dipolos de Comprimentos Diferentes}

Para estudar o caso mais geral de dipolos que têrn comprimentos diferentes, simulamos a situaçäo em que há duas espécies de dipolos definindo o modelo em duas redes diferentes:

$$
L_{1}=d Z^{2} \cap A \quad \text { e } \quad L_{2}=\left[d L Z^{2}+\left(\frac{d}{2}, 0\right)\right] \cap \Lambda
$$

Note que não havera sobreposição se $d>2 L+2$.

$O$ limite superior para a função de correlação, nesse caso em que o gás possui dipolos de comprimento unitário, como antes, e comprimento $L$, é dado por

$$
G(x, y) \leq C_{g} e^{-m \operatorname{tog}|x-y|}
$$

onde $C_{p}<\infty$

$$
m=\frac{p}{8 \pi}\left(1+\beta \frac{z_{1}+z_{2}}{d^{2}} \text { const. }\right)^{-1}
$$

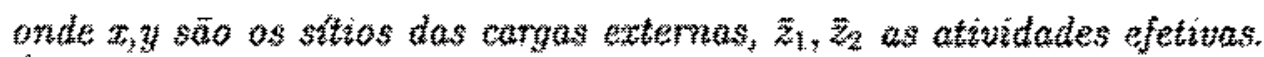

É mahor escrever anteraçao dipolar (3.2) na forma mais geral

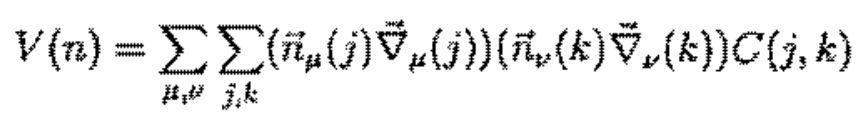

where $C(j, k)$ e dada em $(3.4)$, e o momento de dipolo no sítio $j$ passa a ser $\vec{n}_{k}(j)=\left(n_{k t}(j), n_{k, 2}(j)\right), \vec{n}_{1}^{2}(j)=1, \vec{n}_{2}^{2}(j)=L$.

O potencial efetivo das cargas fracionárias e mesmo dado em (3.5) com

$$
Z(x, y)=\sum_{n=\left\{\vec{n}_{\mu}(j)\right\}} e^{-\frac{\mu}{2} V(n+k)} \prod_{j \in L_{1}} \lambda\left(\vec{n}_{1}(j)\right) \prod_{j \in L_{2}} \lambda\left(\vec{n}_{2}(j)\right) .
$$

correspondendo $a(3.6)$, onde $\mathcal{Z}=\mathcal{Z}(x, y)$ para $\xi=0$ e $\xi(j)=\xi\left(\delta_{j, x}-\delta_{j, y}\right)$. Dipolos de mesmo comprimento tem a mesma atividade distribuição

$$
\begin{aligned}
& \lambda\left(\vec{n}_{1}\right)=\delta\left(\vec{n}_{1}\right)+z_{1} \delta\left(\left|\vec{n}_{1}\right|^{2}-1\right) \\
& \lambda\left(\vec{n}_{2}\right)=\delta\left(\vec{n}_{2}\right)+z_{2} \delta\left(\left|\vec{n}_{2}\right|^{2}-L\right)
\end{aligned}
$$

Agora temos duas densidades de carga

$$
\sigma_{\mu}(j)=\sum_{k, \alpha} n_{\mu \alpha}(j)\left(\delta_{j, k}-\delta_{j, k+\ell_{\mu \alpha} c_{\alpha}}\right) \quad \mu, \alpha=1,2,
$$


e podemos sscrever a função de correlaçăo como

$$
G(x, y)=\sum_{\sigma_{\mu}=\left\{\sigma_{\mu}(j), \mu=1,2\right\}} e^{-\frac{\beta}{2} Y\left(\sigma_{1}+\alpha_{2}+\xi\right)} \prod_{j, \alpha \in L_{1}} z_{\alpha \alpha}(j) \prod_{j, \alpha \in L_{z}} z_{2 \alpha}(j)
$$

novamente $z_{\mu \alpha}(j)=z_{a}(j)^{\left|n_{\mu \alpha}(j)\right|}, \mu=1,2$ (Compare com $\left.(3.9)\right)$

\subsection{Renormalização em $L_{1}$ e $L_{2}$}

A renormalizaçâo dos dipolos em $L_{1}$ é idêntica à anterior (3.7), com a atividade satisfazendo as estimativas (3.10) e (3.11), mesmo quando $d>1$.

Obtemos

$$
\mathcal{Z}(x, y)=\sum_{\tilde{\sigma}_{1}, \sigma_{2}} e^{-\frac{\xi}{2}\left\{\tilde{\sigma}_{1}+\sigma_{2}+\xi_{1}-\Delta^{-1}\left(\bar{\sigma}_{2}+\sigma_{2}+\xi\right)\right)} \prod_{j, \alpha \in L_{1}} \bar{z}_{1 \alpha}(j) \prod_{j, \alpha \in L_{1,2}} z_{2 \alpha}(j)
$$

Já a renormalizaçäo dos diplos em $L_{2}$ requer mais cuidado. É conveniente neste ponto denotar por $\bar{L}_{1}$ e $\bar{L}_{2}$ a coleção de quadrados $B_{1}(j)$ e $B_{2}(j)$ centrados nos sítios das respectivas rede $L_{1}$ e $L_{2}$ de lados de comprimentos $d \mathrm{e}$ dL. respectivamente.

Para cada $j \in \mathrm{L}_{2,}$ fazemos a translaçäo

$$
\bar{\sigma}_{2}=\sigma_{2}+\Delta t_{i}
$$

onde

$$
f_{j}(k)=\left\{\begin{array}{l}
\zeta_{j}(k) g(k) \text { for } k \in \bar{L}_{1} \\
\zeta_{j}(l) g(h),|k-l| \leq 2 \text { for } k \in L_{2}
\end{array}\right.
$$

$\operatorname{com} g(k)=C(j, k)-C(j+L e, k) \mathrm{e}$

$$
\zeta_{j}(k)= \begin{cases}1 & \text { if }|j-k| \leq \frac{3}{2} L \\ 0 & \text { if }|j-k| \geq 2 L\end{cases}
$$

tendo um decaimento suave

$$
\left|\nabla \zeta_{j}(k)\right| \leq \frac{\text { const }}{L} \quad 0 \leq \zeta_{j}(k) \leq 1 .
$$


A função $\zeta_{j}(k)$ permite deslocar a carga dos dipolos em $L_{2}$ sem afetar os dipolos em $L_{1}$, ja que funçoes fora da faixa $\{|k|>2 L\}$, a partir de um ponto em $L_{2}$, nāo se alteram, o o dipolo mais próximo a $j$ está a uma distância maior que $2 L+2$.

Depois da translação escrèvemos

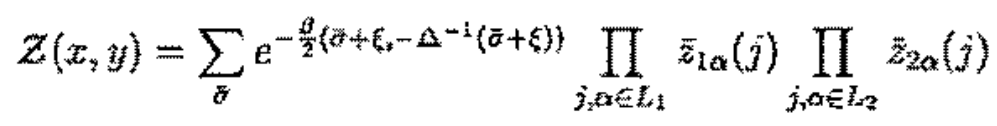

onde $\tilde{\sigma}=\tilde{\sigma}_{1}+\bar{\sigma}_{2}+\xi$.

Agora

$$
\bar{z}_{2 \alpha}(j) \leq e^{\left.-\frac{g}{2}\left(2\left(\sigma_{2} ;\right)\right)-\left(f_{j}-\Delta J_{j}\right)\right)}
$$

Temos as estimativas

$$
\begin{aligned}
\left(\sigma_{2}, f_{l}\right) & \leq f_{j}(j)-f_{j}\left(j+L_{2} e_{n}\right) \\
& \leq \frac{1}{2 \pi} \log L
\end{aligned}
$$

e

$$
\left(f_{j,}-\Delta f_{j}\right) \leq \frac{1}{2 \pi} \frac{\log x}{a^{2}}
$$

onde o lado direito em cada um das estimativas acima pode ser verificado para o caso particular em que $j=0$.

\subsection{Translação}

Falta-nos transladar as cargas externas:

$$
\rho=z+\frac{\gamma}{2 \beta}(\Delta a)(j)
$$

onde agora $a(j)=g(j, x)-g(j, y)$, e $\gamma$ é uma nowa funçầ de $\beta$ para este caso.

A funçăo de partição muda para (Compare com (3.20))

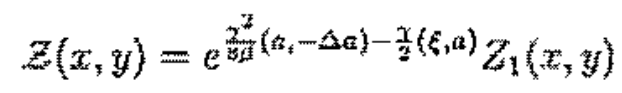

onde, como em (3.18).

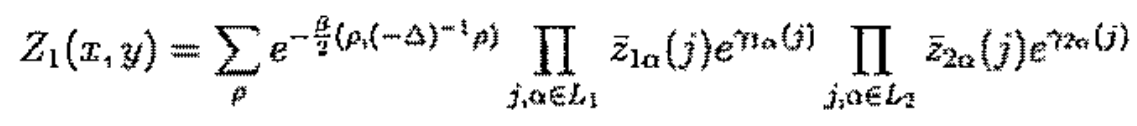


e

$$
\gamma_{1 \alpha}(j)=n_{1 \alpha}(j) \delta_{1} \bar{a}_{\alpha}(j) \quad \gamma_{2 \alpha}(j)=n_{2 \alpha}(j) \delta_{2} \bar{a}_{\alpha}(j)
$$

são análogas a (3.19).

\subsection{Expansões de Cluster}

As expansões de cluster feitas aqui são idênticas à da seção 3.5, exceto que temos de trabalhar um pouco mais antes de aplicá-las.

Para derivar um limite superior para a função de partição (3.37) escrevemos

$$
\prod_{j, \alpha} \bar{z}_{\alpha}(j) e^{\gamma_{\mu \alpha}(j)}=\prod_{j, \alpha} \bar{z}_{\alpha}(j) \cosh \gamma_{\mu \alpha}(j)+R_{\mu}
$$

onde

$$
R_{\mu}=\prod_{j, \alpha}\left(\bar{z}_{\mu \alpha}(j) \cosh \gamma_{\mu \alpha}(j)+\bar{z}_{\alpha}(j) \sinh \gamma_{\mu \alpha}(j)\right)-\prod_{j_{1}} \bar{z}_{\mu \alpha}(j) \cosh \gamma_{\mu \alpha}(j)
$$

para $\mu=1,2$.

Assim (3.37) fica

$$
\begin{aligned}
Z_{1} & =\sum_{\rho} e^{-\frac{\beta}{2} E(\rho)} \prod_{j, \alpha} \bar{z}_{\alpha}(j) \cosh \gamma_{\mu \alpha}(j) \prod_{j, \alpha} \bar{z}_{\alpha}(j) \cosh \gamma_{\mu \alpha}(j)+R_{1} R_{2} \\
& \left.+R_{1} \prod_{j, \alpha} \bar{z}_{\alpha}(j) \cosh \gamma_{\mu \alpha}(j)+R_{2} \prod_{j, \alpha} \bar{z}_{\alpha}(j) \cosh \gamma_{\mu \alpha}(j)\right)
\end{aligned}
$$

Pelas mesmas considerações de (3.27), (3.28) e 3.29), os termos em $R$ de (3.39) podem ser desprezados:

$$
Z_{1} \leq K_{\beta} \sum_{\rho} e^{-\frac{\beta}{2} E(\rho)} \prod_{j, \alpha \in L_{1}} \bar{z}_{1 \alpha}(j) \cosh \gamma_{1 \alpha}(j) \prod_{j, \alpha \in L_{2}} \bar{z}_{2 \alpha}(j) \cosh \gamma_{2 \alpha}(j)
$$

com alguma constante positiva $K_{\beta}$.

Agora desenvolvemos uma expansāo de cluster para $\prod_{j, \alpha} \cosh \gamma_{\mu \alpha}(j) \mu=1,2$ da seguinte forma

$$
\prod_{j, \alpha}\left[1+\left(\cosh \gamma_{\mu \alpha}(j)-1\right)\right]=\prod_{\alpha}\left(1+S_{\mu \alpha}\right)
$$


onde (veja $(3.20)$

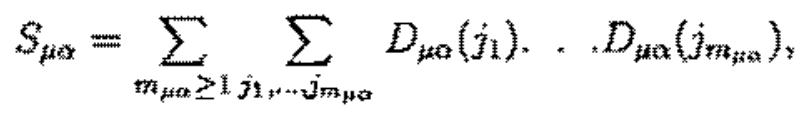

$\operatorname{com}$

$$
D_{\mu \alpha}\left(j_{i}\right)=\cosh \gamma_{\mu k t}\left(j_{i}\right)-1
$$

Expandindo (3.41) obtemos

$$
\prod_{j \alpha}\left[1+\left(\cosh \gamma_{\mu \alpha}(j)-1\right)\right]=1+S_{\mu 1}+S_{\mu \alpha 2}+S_{\mu 1} S_{\mu 2}
$$

Inserindo $(3.44)$ na funç̧ăo de partição $(3.40)$, obtemos

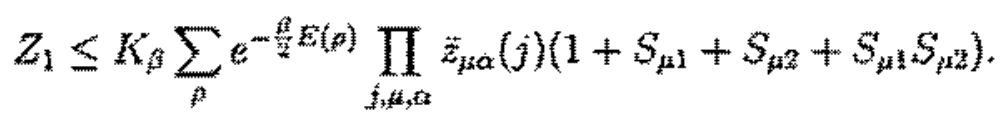

Agora escrevemos

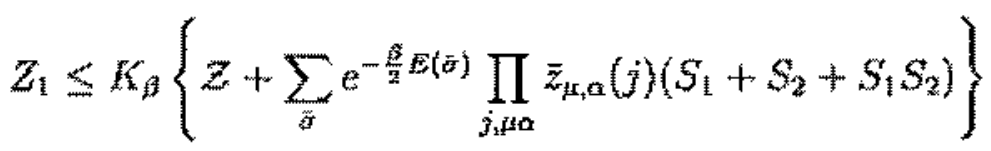

where $S_{\mu}=S_{p k 1}+S_{\mu 2}+S_{\mu 1} S_{\mu 2}, \mu=1,2$.

Claramente $(3.46)$ admite a estimativa

$$
Z_{1} \leq K_{b}\left\{Z+\sum_{\bar{a}} e^{-\frac{B}{2} E(\tilde{a})} \prod_{j, n ; a} z_{\alpha}(j)\left(s_{1}+s_{2}+s_{1} s_{2}\right)\right\}
$$

onde $s_{k 1}=s_{k 1}+s_{i z 2}+s_{k 1} s_{4 k 2}$ e, como em $(3.42)$

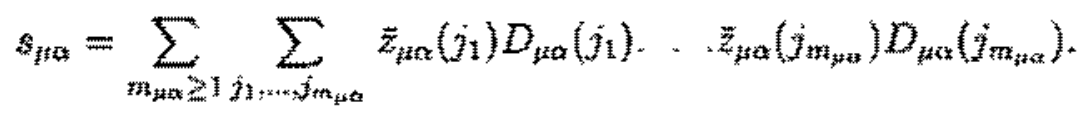

\section{Temos}

$$
\begin{aligned}
z_{1} & \leq K_{\beta} z\left(1+s_{1}+s_{2}+s_{1} s_{2}\right) \\
& \leq K_{\beta} z\left(1+s_{11}+s_{12}+s_{11} s_{12}\right)\left(1+s_{21}+s_{22}+s_{21} s_{22}\right)
\end{aligned}
$$

onde tomamos os termos em $s$ em (3.48) com $n_{\text {tak }}(j)=1$ for all $j_{1} \mu$ e $\alpha$, e usamos que $z>1$. 
De novo desfazemos a expansăo de cluster (3.48) de acordo com as fórmulas (3.41), (3.42) para obter

$$
z_{1} \leq K_{g} z \prod_{j, a \in L_{1}}\left(1+z_{1 a}(j) D_{\mathrm{ka}}(j)\right) \prod_{j, \alpha \dot{\alpha} L_{z}}\left(1+z_{2 \alpha}(j) D_{2 a}(j)\right)
$$

com $n_{\mu \alpha}(j)=1$ para todo $j, \mu$ e $\alpha$

Assim, substituindo a expressäo para $Z_{1}$ ern $(3.36)$, obtemos

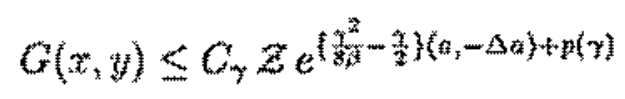

onde

$$
p(\gamma)=z_{4}\left(1+\epsilon_{1}\right) \frac{\gamma^{2}}{2} \sum_{i}\left(\delta_{1} a(j)\right)^{2}+\bar{z}_{2}\left(1+\epsilon_{2}\right) \frac{\gamma^{2}}{2} \sum_{j}\left(\delta_{2} a(j)\right)^{2}
$$

As constantes $\epsilon_{1,} \epsilon_{2}$ yèm do (3.29).

Ambos as somas acima podem ser estimadas pelo mesmo fator

$$
\sum_{j}\left|\delta_{\mu} a(y)\right|^{2}=\text { const } \frac{\log |x-y|}{\pi d^{2}}
$$

O coeficiente da exponencial em $(3.50)$ é

$$
\frac{\gamma^{2}}{8 \beta}-\gamma / 2+\left\{\frac{\gamma^{2}}{2}\left(1+\epsilon_{1}\right) z_{1}+\frac{\gamma^{2}}{2}\left(1+\epsilon_{2}\right) \bar{z}_{2}\right\} \frac{\text { const. }}{d^{2}}
$$

o qual toma o valor ótimo para

$$
2 \beta \gamma^{-1}=1+4 \beta\left\{\left(1+c_{1}\right) z_{1}+\left(1+c_{2}\right) z_{2}\right\} \frac{\text { const }}{d^{2}} .
$$

Entäo obtemos

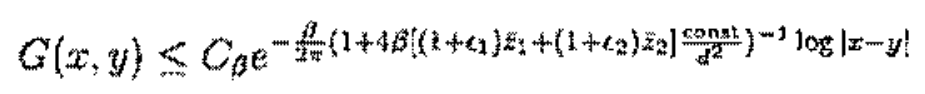




\section{Capítulo 4}

\section{O Modelo de Villain}

Neste capitulo provamos que a fase dipolar do gás de Villain é caracterizada por

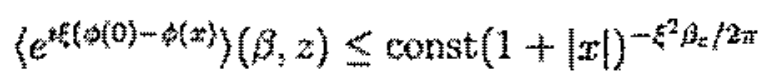

para algum $\beta_{s}(\beta, \xi, z)$.

Reproduzimos o resultado de [20] quanto is estimativas, mas usamos 0 método de multi-escala como em [30].

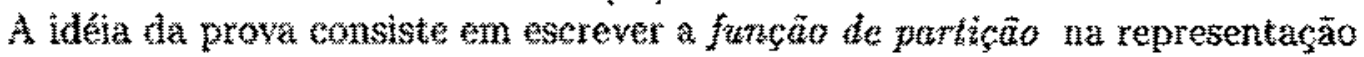
de sine-Gordon (ver 2.7)

$$
Z\left(\sigma_{2}\right)=\int \prod_{z \in \hat{R}} \hat{\lambda}\left(\hat{\phi}+\sigma_{2}\right)(x) d \mu_{\beta}(\phi)
$$

onde

$$
\left.\hat{\lambda}\left(\phi+\sigma_{2}\right)(x)=1+2 \sum_{q=1}^{\infty} \lambda(0) \cos \left(\phi(x)+\sigma_{2}(x)\right) q(x)\right)
$$

como combinaçäo convexa de funçöes de partição de gases de multipolos neutros.

Cada passo do processo consiste em construir ensembles esparsos, o que significa diminnir a densidade do gás. Sabemos que uma alteraçāo na densidade provoca uma mudança na atividade do gás. No caso, estaremos aumentando a atividade. Esse é o custo entrópico do processo. Por outro lado, para que a medida de probabilidade na variável $\phi$ seja positiva - ou equivalentemente -.. a sona em (4.1) convirja, a atividade do gás não poderá ser maior do que 1. Logo deveremos compensar o custo entrópico pela extraçāo da auto-energia 
das cargas de cada ensemble, a qual terá de superar o aumento de entropia resultante do agrupamento de cargas em blocos esparsos.

\subsection{Combinações Convexas}

Aqui começamos a aplicar as técnicas de renormalização para escrever a função de partição como combinações convexas de funções de partição regulares. Como em [25], escolhemos uma sequência $\left\{\zeta_{q(x)}\right\}_{q(x)=1}^{\infty}$ de números positivos, cuja dependência com $q(x)$ há de ser posteriormente estabelecida, satisfazendo a condiçāo

e escrevemos

$$
\sum_{q(x)=1}^{\infty} \zeta_{q(x)}=1
$$

$$
\prod_{x} \hat{\lambda}\left(\phi+\xi \sigma_{2}\right)(x)=\sum_{q_{\Lambda}} \zeta\left(q_{\Lambda}\right) \prod_{x}\left[1+z(q(x)) \cos \left(\left(\phi(x)+\sigma_{2}(x)\right) q(x)\right)\right]
$$

onde

$$
\zeta_{q_{\Lambda}}=\prod_{x \in \Lambda} \zeta_{q(x)} \quad e \quad q_{\Lambda}=\{q(x)=1,2,3, \ldots ; x \in \Lambda\} .
$$

e $z(q(x))=2 \lambda(q(x)) / \zeta_{q(x)}$.

A sequência $\zeta_{q}$ pode ser escolhida arbitrariamente, contanto que

$$
\left|\zeta_{q}\right|^{-1} \leq \text { conste } e^{c q^{2}}
$$

com $0<c<\infty$.

Note que $\sigma(x)=\xi(-\Delta)^{-1} \partial_{2} f^{x}$ nāo será afetado pela renormalização de cargas, e será pequeno se $\rho$ for neutro e tiver suporte longe de 0 ou $x$.

Temos que $\mathcal{Z}\left(\sigma_{2}\right)$ será combinação convexa de funções da forma

$$
\mathcal{Z}\left(\sigma_{2}\right)=\sum_{q_{\Lambda}} \zeta\left(q_{\Lambda}\right) \int \prod_{x}\left[1+z_{x} \cos \left(\phi(x)+\sigma_{2}(x)\right) q(x)\right] d \mu_{\beta}(\phi)
$$




\subsection{A Construção dos Blocos}

A seguir, fixamos a geometria do problema. Dividimos a rede em blocos. Denotamos por $B(x, L)$ o quadrado em $Z^{2}$ de lado $L$ com centro em $x$

$$
B(x, L)=\left\{y \in Z^{2}:|z-y|<L / 2\right\}
$$

tal que

$$
A=\bigcup_{y \in A} B_{0}(y, L) \quad \hat{A}=A \cap 3 Z^{2}
$$

Fixado $q_{h}$

$$
\prod_{x \in \hat{\Lambda}}\left(1+z(q(x)) \cos \left(q(x) \phi^{\prime}(z)\right)\right)=\prod_{y \in \hat{A} u \in B_{n}(y)}\left(1+z(q(u)) \cos \left(q(u) \phi^{z}(u)\right)\right.
$$

onde $\phi^{\prime}=\phi+\sigma$.

Seguindo [30] reapresentamos o [Lemma 2.1], com o qual o produto acima é escrito como combinação convexa de fatores similares correspondentes a multipolos de dimensẫo variável.

Lema 4.1 Seja $z_{i}>0, \phi_{i}^{t} \in \mathbf{R}, i=1,2, \ldots, N$. Expandimos o produto acima eru soma de produtos por meio da identidades

$$
\prod_{i=1}^{N}\left(1+z_{i} \cos \phi_{i}^{\prime}\right)=\sum_{\tau \in g N} c_{\tau}\left(1+z_{i} \cos \phi^{\prime}(r)\right)
$$

onde $g_{t}\{\tau: 1,2, \ldots, N \rightarrow 0,1,-1, \tau \equiv 0\}, \phi^{\prime}(\tau)=\sum_{i=1}^{N} \phi_{i} \tau_{i}, 0<c_{r}$ $\sum_{\mathrm{rE} E_{N}} c_{\tau}=1, \mathrm{e}$

$$
z_{r}=\prod_{i=1}^{N}\left(b_{N} z_{i}\right)^{\left|r_{i}\right|}
$$

Note-se que a magnitude do coeficiente $c_{T}$ năo é importante; mas sim seu sinal, $c_{r}>0$. A variável de bloco $b_{N}$ depende unicamente do número $\mathrm{N}$ de elementos do conjunto $\tau_{y}$, com a única condiçẫo de que

$$
\sum_{\tau_{y}} c_{r_{y}}=\sum_{\gamma_{y}}\left(\frac{1}{2 b_{N}}\right)^{\left|\gamma_{y}(x t)\right|}=1
$$

\footnotetext{
${ }^{1}$ Usamos a identüdade: $\prod_{i=1}^{N} \cos \phi_{i}^{t}=(1 / 2)^{N} \sum_{\{+\}} \cos \left(\sum_{i=1}^{N} \pi_{i} \phi_{i}^{t}\right) \operatorname{com} t_{i}= \pm 1, i=$ $1,2, \ldots, N$
} 
Essa condiçāo determina unicamente a constante $b_{N}$ visto que

$$
1=\sum_{7_{y}}\left(\frac{1}{2 b_{N}}\right)^{\left|w_{y}(w)\right|}=\sum_{n=1}^{N} \frac{1}{b_{N}}=\left(1+\frac{1}{b_{N}}\right)^{N}-1
$$

ou

$$
b_{N}=\left(2^{1 / N}-1\right)^{-1} \leq \frac{N}{b_{N}}
$$

Disso resulta a estimativa

$$
z_{y} \leq \prod_{u \in B_{u}(y)}\left(\frac{9}{\log 2} \frac{2 \lambda\left(\rho_{y}(u)\right)}{\zeta_{\rho_{y}}(u)}\right)
$$

(Em nosso caso, $\left.\left|B_{0}(y)\right|=9.\right)$

Esse lema é aplicado em cada $y \in \hat{\Lambda}$ para escrever (4.3) como combinaçāo convexa de funçôes do tipo

$$
\int \prod_{y \in \mathbb{A}}\left[1+z_{y} \cos \left(\phi\left(\rho_{y}\right)+\sigma_{2}\left(\rho_{y}\right)\right)\right] d u_{b}(\phi)
$$

Aqui

$$
\phi(p)=\sum_{u \in B_{0}(y)} \phi(u) \rho(u)
$$

\subsection{Reprimindo a Atividade}

Para $\beta$ suficientemente grande, o gás de Coulomb comportar-se-á como gás de multipolos em que grandes multipolos serão improvŭveis se a atividade efetiva em funçăo de $\beta$ for pequena. Visando a reprimir a atividade $z_{\sigma_{y}}$ das eargas do bloco $B_{0}(y)$, gostariamos de extraír o termo de auto-energia de $E\left(g_{n}\right)$ mediante a seguinte desigualdade eletrostatica. Isso é feito pela translação complexa $\phi \rightarrow \phi+\imath a, a \in \mathbf{R}_{\text {: }}$ usando o seguinte lema.

Lema 4.2 Seja $\rho: \mathbf{Z}^{2} \rightarrow \mathbf{R}$ com suporte compacto, e seja $G(\rho)$ um funcional independente de $\phi(i): i \in$ suppp. Então

$$
\int e^{z \phi(\phi)} G(\phi) d \mu_{\beta}=e^{-\beta\left(\rho(\beta, a)-\frac{1}{2}\left(a_{n}-\Delta_{n}\right)\right)} \int e^{z \phi(\phi+\Delta a)} G(\phi) d \mu_{\beta}
$$


A renormalizaçầo da densidade de carga se faz como em $(3,7)$ :

$$
\tilde{\rho}=p+\frac{1}{\|\Delta\|} \Delta \rho
$$

O laplaciano, porĕ̉m, deslocaliza as cargas efetivas do bloco original, podendo com isso ocorrer em $\bar{q}_{A}$ uma superposiçào de cargas inicialmente em blocos distintos. Evitamos esse efeito observando que a desigualdade eletrostática é valida ainda quando a transformaçäo de cargas seja efetuada apenas num subconjunto de $q_{A}$ - cuja escolha será especificada em conformidade com a construção de blocos que impusermos. Com isso, formamos blocos maiores que contenham alguns $B_{0}(y)$ e selecionamos - a seleção näa é única - em cada um desses blocos um ponto para o qual seja lícito aplicar a desigualdade eletrostática sem deslocar as cargas escolhidas para fora da fronteira comum. O ponto selecionado, $u_{y}$, aquele em que

$$
p_{y}^{2}\left(t_{y}\right) \geq \frac{1}{9} \sum_{u \in B_{g}(y)} p_{y}^{2}(u)
$$

Os blocos agrupados definem uma equivalência segundo o critério

$$
y \sim y^{*} \leftrightarrow\left|u_{y}-u_{y}\right|=1
$$

e o agrupamento, a classe de equivalência $Y$ :

$$
B_{0}(Y)=\bigcup_{y \in Y^{Y}} B_{0}(y)
$$

Cumpre observar que há quatro formas possiveis para eada $Y$, conforme $|Y|=1,2,3$ ou 4 . Esse procedimento vai garantir que, em cada bloco, o gás seja suficientemente diluto à fim de ser possível extrair a porção local da auto-energia eletrostática exp[const $\beta E(q)]$ das cargas $u_{y}$. Em termos dos novos blocos

$$
\prod_{y \in \hat{A}}\left(1+z_{y} \cos \phi^{\prime}\left(\rho_{3}\right)\right)=\prod_{Y} \prod_{y \in Y^{Y}}\left(1+z_{y} \cos \phi^{\prime}\left(\rho_{y}\right)\right)
$$

A repetição da transformação do produto dos termos de $B_{0}(y), y \in Y$ em soma convexa sobre um conjunto $\tau$ cujos elementos são funçöes constantes 
nos blocos constituintes de $\mathrm{Y}$, com valores possiveis em cada um deles iguais a $0, \pm 1$, conduz a

$$
\mathcal{Z}=\sum_{n_{\Lambda}} \zeta_{n_{\Lambda}} \sum_{\tau} c_{\tau} \sum_{\omega} c_{\omega} Z_{n_{\Lambda}, r, \omega}
$$

agora,

$$
Z_{n_{A_{1}} t_{i}, \omega}=\prod_{Y}\left(1+z_{Y} \cos \phi^{\prime}\left(p_{Y}\right)\right)
$$

correspondentemerte $Y$ possui a distribuigâo de cargas compatível com a dos blocos internos:

$$
p(u)=w_{y} p_{y}(z), \quad y \in B_{0}(Y)
$$

O bloco $Y$ que maximiza a atividade é o de $|Y|=4 \operatorname{com}\left|\omega_{y}(u)\right|=1$ para todos os quatro pontos y internos, resultando na estimativa:

$$
0<z_{Y} \leq\left(\frac{4}{\log 2}\right)^{4} \prod_{u \in B_{u}(Y)}\left[\frac{9}{\log 2} z_{w\{u)}\right]
$$

Neste ponto, aplicamos a desigualdade eletrostática restrita ao conjunto de pontos $U_{Y}=\left\{u_{y}, y \in Y\right\}$ cujas cargas efetivas säo $\tilde{p}_{Y}$ com o fito de reprimir a atividade $z_{Y}$ do bloco $Y$ pelo fator de auto-energia das cargas em $U_{Y}$ :

$$
z_{Y}=z_{Y} \exp \left\{-\frac{\beta}{16} \sum_{y \in U_{Y}} p_{y}^{2}\left(a_{y}\right)\right\}
$$

Pelo critério de seleção dos pontos selecionados daquele conjunto, segue a estimativat

$$
z_{Y} \leq z_{Y} \exp \left\{-\frac{\beta}{144} \sum_{u \in B_{0}(Y)} p^{2}(u)\right\}
$$

Para qualquer $Y$, as atividades säo limitadas pela funçăo de $\beta$

$$
\bar{z}_{Y} \leq K_{0}(\rho)=\left(\frac{4}{\log 2}\right)^{4} \frac{18}{\log 2} \sup _{q=1,2,3, \ldots}\left[\frac{\lambda(q)}{\zeta_{q}} e^{-\frac{g}{\beta_{q} q^{2}}}\right] .
$$

\subsection{Um Passo Intermediário}

$O$ inconveniente com os blocos $Y$ sāo suas diferentes formas. A maneira de recuperarmos os blocos quadrados é passarmos a uma escala superior que 
agrupe todas as quatros formas possiveis de cada $Y$, mediante o seguinte procedimente:

1) Definimos uma coleção de densidades de carga cujos suportes são mutuamente disjuntos;

2) As áreas de densidades de carga são quadrados $\Lambda^{(n)}=\Lambda \cap L_{1} Z^{2}$ de lado $L_{1}=3^{n_{1}}\left(n_{1} \geq 3\right.$, inteiro $)$, necessários para cobrir o suporte dessas densidades;

3) O critério de localizaçâo das densidades de carga em $B\left(y, L_{1}\right)$ é

$$
\text { sutpp } \rho \subset B\left(y, \frac{4}{3} L_{1}\right)=\bar{B}\left(y, L_{1}\right)
$$

$\mathrm{Na}$ escala assim definida, temos a seguinte igualdade para cada par $(\rho, z(\rho))$ de densidade de carga e da respectiva atividade localizado no quadrado $B\left(y, L_{1}\right):^{2}$

$$
\int \prod_{\left.y \in x^{2}\right)}\left[1+z_{y} \cos \left(\phi\left(\tilde{p}_{y}\right)+\alpha_{2}\left(p_{y}\right)\right) d u_{2}(\phi)\right.
$$

onde $\Lambda^{(1)}=\Lambda \cap L_{1} Z^{2}$, tom $L_{1}=3^{n+}, \odot n_{1}>3$ é um inteiro. Agora a densidade de carga esta localizada em $B_{1}(y)=B\left(y, L_{1}\right)$, com

$$
z\left(\rho_{y}\right) \leq C_{1} \prod_{u \in B_{1}(y)}\left[\frac{9}{\log 2} \frac{2 \lambda\left(\rho_{y}(u)\right)}{\zeta\left(\rho_{y}(u)\right)}\right]
$$

e $C_{1}=(1 / \log 2)^{5}\left(64 / 9 L_{1}\right)^{2}$. O valor de $z_{0}$ depende das formas dos blocos constituintes das classes de equivalència. O máximo valor ocorre quando esses blocos sào todos iguais e tền $|Y|=1$, do modo que ternos somente um $t_{y}$ em cada $Y$, perfazendo o número total

$$
\frac{1}{9}\left(\frac{4}{3} L_{1}\right)^{2}
$$

de pontos em cada $\Lambda^{(1)}$. Correspondentemente temos a estimativa:

$$
z_{u^{*}} \leq\left(\frac{4}{9} L_{1}\right)^{2} \frac{K_{0}(\beta)}{\log 2}=K_{1}\left(L_{1}, \beta\right)
$$

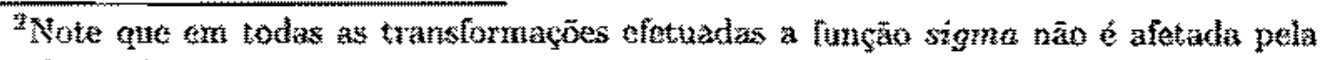
translaçẩo de catga.
} 
Ao concluirmos essa sequencia de transformações , a função de partiçăo de um gảs de Coulomb bidimensional está escrita como combinação convexa de funçöes de partição de um gás de multipolos da forma (4.5). Essas são as condiçóes iniciais para a realização de um processo indutivo sobre a função de partiçäo na ausência de cargas externas apenas. Tanto o numerador quanto o denominador podem ser tratados da mesma maneira.

\subsection{O Procedimento Indutivo para a Função de Partição}

Aqui continuamos o processo de renormalização de densidades de cargas e atividades cujo objetivo é escrever a funçāo de partição cono combinação convexa de funçōes de partição regulares, que serão definidas em seguida, nas escalas de $\rho$.

Dado $n_{1} \geq 3$, e construida a primeira escala de lado $L_{1}=3^{n_{3}}$; a funçäo de partiçầo do gás de Coulomb está escrita cono combinaç̧o convexa de expressoes da forma $(4.5)$

$$
\int \prod_{y \in A}\left(1+z\left(\bar{\beta}_{y}\right) \cos \left(\phi\left(\bar{\beta}_{y}\right)+\sigma_{z}\left(\rho_{y}\right)\right)\right) d u_{y}(\phi)
$$

com atividade $K_{1}\left(L_{1}, \beta\right)<1$.

Para $k=1,2,3, \ldots$, vamos construir funçöes, chamadas funçōes de partiçāo regulares, que obedecem a uma relação de recorrância entre as escalas. Será possivel, entäo, identificar a função de partição do gás como combinaçäo convexa, em cada escala, de funçōes de partição regulares, a partir de sua identificaçäo com com as funções regulares da primeira escala. Com isso, poderemos propagar as estimativas das atividades extraindo os termos de auto-energia dos elementos carregados em cada escala pela aplicaçăo da desigualdade eletrostática a eles.

As escalas usadas são da forma $L_{k+1}=L_{k,}^{\alpha} \operatorname{com} \alpha>1$. Fixando uma delas, queremos que na rede $B\left(y, L_{k}\right)=B_{k}(y)$, formada por blocos de lado $L_{k}$, a função de partiç̧ăo do gás seja escrita como combinação convexa de funçöes indexadas pelos parâmetros $(k, r)$, chamadas funçōes de partiçầo regulares, 
e definidas por

$$
Z_{(k, r)}=\int F\left(\mathcal{N}_{(k, y, r)} ; \phi\right) \prod_{y \in \Lambda^{(k)}}\left[1+z\left(\rho_{y}\right) \cos \left(\phi\left(\bar{\rho}_{y}\right)+\sigma_{2}\left(\rho_{y}\right)\right)\right]
$$

As densidades de carga $\rho_{y}$ localizadas em $B_{k}(y)$ para todo $y \in \Lambda^{(k)}$ com supp $\rho_{y} \subset \bar{B}\left(y, L_{k}\right)$ satisfazendo $z\left(\rho_{y}\right) \leq L_{k}{ }^{-r}$, são chamadas densidades admissíveis de carga $(k, y, r)$.

Seguindo a notação de $[30]$, temos

i) $\overline{\rho_{y}}$ está localizada em $B\left(y, \frac{4}{3} L_{k}\right)$

ii) $\rho_{y}$ está localizada em $B\left(y, L_{k}\right)$

iii) $z\left(\rho_{y}\right) \leq L_{k}^{-t} e^{-C|\rho|^{2}}$

O fator $\mathbf{F}\left(\mathcal{N}_{(k, y, r)}\right)$ é dado por

$$
F\left(\mathcal{N}_{(k, y, r)} ; \phi\right)=\int \prod_{\rho, \bar{\rho} \in \mathcal{N}_{k, y, r}}\left[1+z(\rho) \cos \left(\phi(\bar{\rho})+\sigma_{2}(\rho)\right)\right] d \mu_{\beta}(\phi)
$$

onde $\left\{\mathcal{N}_{k, y, r}, \rho_{y}, \bar{\rho}_{y}, z_{y}\right\}$ é uma coleção de cargas, e $\rho=(\rho, \bar{\rho}, z(\rho))$ é uma densidade de carga admissivel $(k, y, t)$.

Nessa expansão, todo $\rho$ em $\mathcal{N}_{k, y, r}$ é neutro e separado de outros.

Esse fator estabelece um vínculo com as escalas anteriores pois depende das coleções de densidades de carga $\mathcal{N}_{(k, y)}$ nelas contidas. Essas coleções reúnem as cargas das escalas até $k-1$ iterativamente por

$$
\mathcal{N}_{(k, y)}=\left[\bigcup_{y^{\prime} \in B\left(y^{\prime}, L_{k}\right)} \mathcal{N}_{\left(k, y^{\prime}\right)}\right] \cup\{\rho\}
$$

a partir da primeira escala onde $\mathcal{N}_{(k=1, y)}=\emptyset$. A carga $\rho$ está localizada em $B_{k}\left(y^{\prime}\right)$ para algum $y^{\prime} \in B_{k+1}^{(k)}(y)$ cuja atividade é $z\left(\rho_{y}\right) \leq\left(\frac{8}{\log 2}\right)^{4} L_{k}^{-r}$.

Em face dessas propriedades, a funçāo de partiçāo do gás da primeira escala é identificada com a funçāo de partiçāo regular $(k=1, r+2(\alpha-1))$, para a qual a admissibilidade de seus parâmetros se realiza pela estimativa

$$
K_{1}\left(L_{1}, \beta\right) \leq L_{1}^{-[r+2(a-1)]} .
$$




\subsection{A Hipótese Indutiva}

A hipótese indutiva é a de que, dada a condição inicial $\mathcal{N}_{(k=1, y)}=\emptyset$, qualquer funçăo de partição regular $(k+1, r)$ pode ser obtida das funçöes de partição regulares $(k, r)$ para $k=1,2,3, \ldots, L_{k+1}=3^{n_{k+1}}$ e $n_{k+1}=\left[\alpha n_{k}\right]$.

De fato, se a funçäo de partiçăo regular na escala $k$ é

$$
z_{(k, v)}=\prod_{y \in A^{(k)}} \mathrm{F}\left(\mathcal{N}_{(k, y, n)}\right) \int \prod_{y \in A^{(k)}}\left(1+z\left(p_{y}\right) \cos \left(\phi\left(\vec{\rho}_{y}\right)+\sigma_{2}\left(\rho_{y}\right)\right)\right) d t_{\phi}(\phi)
$$

é possivel passar a escala $k+1$ usando o Lema $4 . \lambda$ para transformar os dois termos do lado direito de $(4.6)$.

O produto

$$
\prod_{y \in x^{(k)}}\left(1+z\left(\hat{\rho}_{y}\right) \cos \left(\phi\left(\hat{\rho}_{y}\right)+\sigma_{2}\left(\hat{p}_{y}\right)\right)\right.
$$

se transforma em combinaçäo convexa de funçōes de partiçẫo na rede de centros em pontos $t \in \Lambda^{(k+1)}$

$$
\prod_{u \in \lambda^{(k+1)}}\left(1+z_{u}^{*} \cos \left(\phi\left(\vec{\rho}_{y}^{*}\right)+o_{z}\left(\rho_{y}^{*}\right)\right)\right.
$$

O termo $F$ por sua vez se transforma em

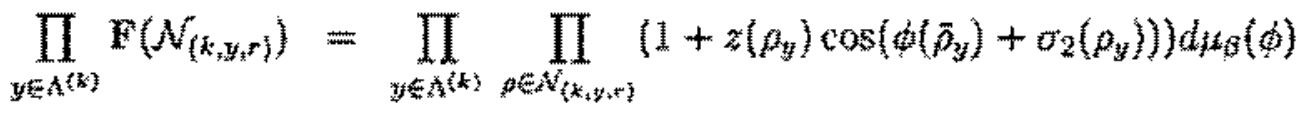

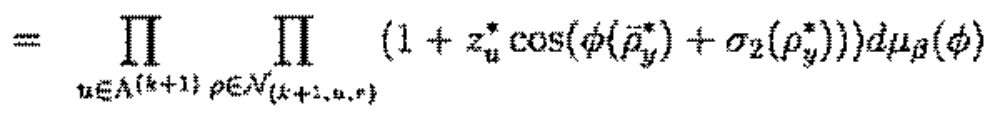

$$
\begin{aligned}
& =\prod_{\mathcal{H} \in N^{k+1}} F\left(N_{(k+1, a, r)}\right)
\end{aligned}
$$

onde

$$
\tilde{N}_{(k+1, z, r)}=\bigcup_{y \in B_{k+1}^{k}(z)} \mathcal{N}_{(k, t, r)}
$$

e

$$
\hat{\rho}_{u}^{*}=\sum_{y \in B_{k+3}^{(k)}\{u)} \tau_{y} p_{y}, \quad \text { e } \rho_{u}^{*}=\sum_{y \in R_{k+1}^{(k)}\{(u)} \tau_{y} \rho_{y}
$$


onde $B_{k+1}^{(k)}(u)=B_{k}(t) \cap L_{k+1} Z^{2}$, e $\tau \in G(L)=\{\tau: T \rightarrow 0,1, \cdots 1\}$.

O par $\left(p^{*}(u), z_{u}^{*}\right)$ é da forma $p^{*}(u)=\tau_{y} p_{y}(u)$ para algum $\tau \in \mathcal{G}\left(A_{k+1}^{(x)}(u)\right)$.

A estimativa para a atividade fica

$$
z_{u}^{*} \leq\left[\frac{1}{\log 2}\left(\frac{L_{k+1}}{L_{k}}\right)^{2} \frac{1}{L_{k}^{r+2(a-1)}}\right]^{\sum_{x}\left|\tau_{y}\right|} .
$$

Substituindo $L_{k+1}=L_{k}{ }^{\circ}$ esulta

$$
z^{*}(a) \leq\left(\frac{1}{\log 2} \frac{1}{L_{k}{ }^{2}}\right)^{\sum_{k}\left|z_{8}\right|}
$$

Todo $\rho$ em $\tilde{\mathcal{N}}$ é neutro, exceto $\rho=\rho^{*}$ que é carregado.

\subsection{Extração da Energia de Cargas Isoladas}

Em alguns casos sera preciso generalizar o procedimento de extrait a aum tomenergia eletrostatica dos coustituintes carregados das densidades do carga

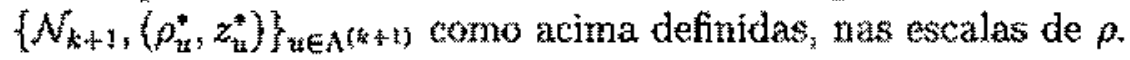

Isso é feito generalizando o Lema 4.2 de deslocamento de cargas para acom modar uma translação das cargas pelo laplaciano de uma função $f_{k}$ em cada escala, à semelhanç do que fizemos para os dipolos.

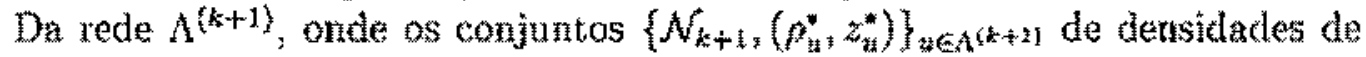
carga se localizam, vamos supor que em am de seus blocos, por axemplo, no

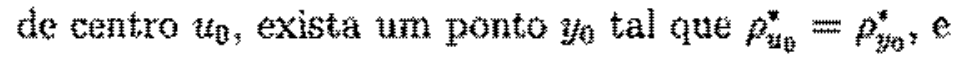

$$
B\left(y_{0}, \frac{1}{3} L_{k+1}\right) \cap \operatorname{sipp} \rho_{1+0}^{*}=0
$$

para todo $u \in \Lambda^{(k+1)}, u \neq u_{0}$.

Admitiremos, por simplicidade, que $\rho_{u_{0}}^{*}=\rho_{0}^{*}$ estat localizado em $B_{k}(0)$. A condição de validade da hipótese acima é supp $\rho_{u}^{*} \cap B\left(0, \frac{1}{3} L_{k+1}\right)=0$ para todo $u_{i} \in A^{(k+1)}$, $\quad$ it 0 . Queremos aplicar a desigualdade eletrostática apenas a $\rho_{0}^{*}$ o que exigira uma melhor demareaço da região $B\left(0, \frac{1}{3} L_{1+1}\right)$ onde está o suporte da carga.

Imaginamos, entăo, que cada funçāo $\rho_{y} \in N_{(x+1,0, r)}$ está localizada numa caixa $B_{n y}(y)$, onde $n_{y}=\inf \left\{n \in \mathbb{N}: \operatorname{supp} p_{y} \in B_{n}(y)\right\}$. 
Com isso, podemos definir no bloco $B_{k}, 1,(0)$, gue contem as caixas, as classes de equivalència distintas $Y_{1, \ldots,}, Y_{\text {p }}$ pela regra

$$
y \sim y^{\prime} \leftrightarrow B_{n_{y}}(y) \bigcap B_{p_{y}}\left(y^{*}\right)=0 \text {. }
$$

A reuniäo das densidudes de carga contidas numa mesma classe de equivalencia forma os conjuntos

$$
B\left(Y_{t}\right)=U w_{n} B_{z_{k}}(y)
$$

$t=1, \ldots, p$

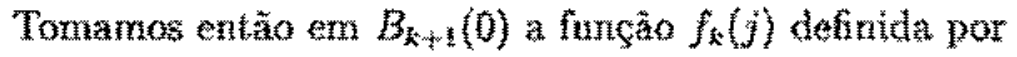

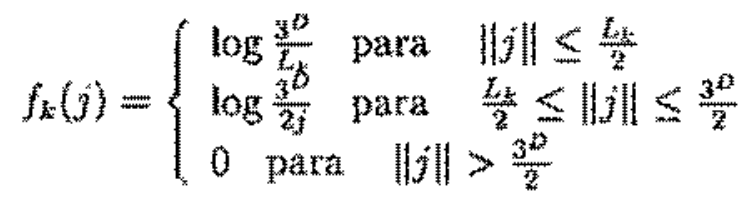

e introduzimos

$$
q=\sum_{v \in B_{*}(0)} p_{0}^{*}(y)
$$

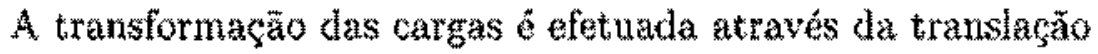

$$
\vec{p}_{A}=p_{A}+\kappa q \Delta \hat{f}_{k}
$$

onde $k \dot{e}$ um parâmetro a ser escolhido posteriormente, e a funçấo $\vec{f}_{k}(j)$ é definida em termos de $f_{k}(j)$ como segue

$$
\hat{f}_{k}(j)=\left\{\begin{array}{l}
f_{k}(j) \text { se } j \in \mathcal{U}_{t=1}^{n} B\left(Y_{t}\right) \\
f_{k}\left(y_{t}\right) \text { se } j \in B\left(Y_{t}\right), \quad t=1, \ldots, p,
\end{array}\right.
$$

para algum ponto $\tilde{w}_{h}$ en $B\left(Y_{t}\right)$.

Da definiçăo de $f_{k}(j)$

$$
\left(p_{k}, f_{k}\right)=\log \frac{3^{D}}{L_{k}}
$$

e, se conseguirmos o limite superior

$$
\left(\Delta f_{k}, f_{k}\right) \leq \delta \log \frac{3^{D}}{L_{k}}
$$


para alguma constante $\delta<\infty$, teremos extraido o fator de decaimento

$$
\left(\frac{L_{k+1}}{3 L_{k}}\right)^{-8 \kappa q^{2}} \leq Y^{(k)} \leq\left(\frac{L_{k+1}}{3 L_{k}}\right)^{-\beta \kappa q^{2}(1-\kappa / 2 \pi)}
$$

De fato, aquele limite de $\left(\Delta f_{k}, f_{k}\right)$ existe. A estimativa $\delta^{-1}=8+O\left(L_{1}^{3+2 \alpha}\right)$ é obtida como en [MKPYO].

Assim, sendo \%o arbitrário, se $\alpha>3 / 2$, para qualquer $\kappa>0$, a funçäo de partiçäo, anteriormente obtida como produto de termos sobre todos os pontos u da rede $\Lambda^{(k+1)}$, agora terá o termo relativo ao ponto $t_{0}$ substituido pelo fator de decaimento $Y^{(k)}$.

Assim, para algum $\tilde{p}_{\mathrm{up}}^{*} \in \Lambda^{(k+1)}$, mantendo $p^{*}$ inalterado, obtemos

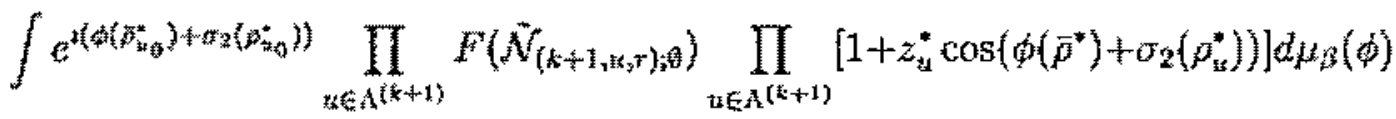

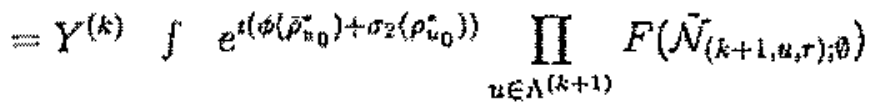

$$
\begin{aligned}
& \prod_{u \in \Lambda^{(k+1)}}\left[1+z_{u} \cos \left(\phi\left(\bar{\rho}^{*}\right)+\sigma_{2}\left(\rho_{u}^{*}\right)\right)\right] d \mu_{\beta}(\phi)
\end{aligned}
$$

onde

$$
q=Q\left(\rho_{u}^{*}\right)=\sum_{y \in B\left(u, \frac{1}{3} L_{k}\right)} \rho_{u}^{*}(y)
$$

e $\hat{o}^{-1}=8+0\left(L_{1}^{3-2 \alpha}\right)$ é independente de $\kappa$, e $\bar{\rho}_{u_{0}}^{*}$ é a densidade de carga localizada em $B\left(w_{0}, \frac{1}{3} L_{k+1}\right)$ tal que $Q\left(\bar{\rho}_{u_{0}}^{*}\right)=Q\left(\rho_{\mathrm{u}_{0}}^{*}\right)$.

Note-se que a transformaçăo das cargas nenhuma alteração acarreta em $\mathcal{F}\left(\tilde{N}_{k+1, i, n, p}\right)$.

O processo descrito acima é levado até a escala $N$. Para completar o prom cesso, construímos um próximo bloco tal que $L_{N+1}=\infty$, de modo que $Y(j) \cdots+1)=0$. Assim, a contribuição de todos os termos contendo fatores $z_{*} \cos \phi\left(p^{*}\right) \operatorname{com} Q\left(\rho_{* 0}^{*}\right) \neq 0$ se anula.

$A$ análise deve acomodax ainda aquelas situaçōes em que não valha a condiçâo $B\left(y, \frac{L}{3} L_{k+1}\right) \cap$ supp $p_{t a}^{*}=0$. Para isso remetemos o leitor a [30].

Terminamos assim o tratamento da funçäo de partição do şás na ausencia de cargas externas, baseando nossa analise na obtenção de combinaf̧ōes convexas da função de partição e na construção de condiçōes apropriadas para 
aplicar a desigualdade eletrostática de modo que reproduzissemos os resultados da referência acima.

Para $3 / 2<\alpha<2,2 \alpha(\alpha-1) /(2-\alpha)<r<\beta \delta / 2-2 \alpha$, a função de partiçăo está escrita cono combinaçāo convexa de funç̧es de partiçảo regulares, com os parâmetros acima, para qualquer $k=1,2, \ldots$ se $L_{1}$ for suficientemente grande.

\subsection{A Função de Correlação das Cargas Ex- ternas}

A expansão acima é teita até que todas as cargas $p$ sejam noutras, ou seja, até que a contribuçăo de todos os termos contendo um fator $z \cos \phi(p)$ com $Q(\rho) \neq 0$ se cancele. Seja $\mathcal{N}$ a coleção de cargas neutras resultantes do procedimento acima, e $Z_{M}\left(\sigma_{2}\right)$ a função de partiçăo regular para aquela coleção de cargas na escala $N$. Temos

$$
z_{N}\left(\sigma_{2}\right)=\int \prod_{p \in N}\left[1+z(\rho) \cos \left(\phi(\bar{\rho})+\sigma_{2}(\rho)\right)\right] d \mu_{p}(\phi)
$$

$\operatorname{com} \mathcal{Z}=\mathcal{Z}_{\mathcal{N}}\left(\sigma_{2}=0\right)$.

O limite inferior para a expressāo acima pode é obtido fatorando a dependência de $\sigma_{2}$ tha funçäo de partiçāo, como em [25].

Definimos

$$
x=z(\rho) \cos \phi(\bar{\rho}) \quad y=z(\rho) \cos \left(\phi(\ddot{\rho})+\alpha_{2}(\rho)\right)-x
$$

e escrevemos

$$
0<1+z(p) \cos \left(\phi(p)+o_{2}(p)\right)=\exp \ln (1+x+y)
$$

Expandindo o logaritmo em torno de $y=0$, usando o teorema de Taylor com resto até segunda ordem em $y$, vem

$$
1+z(\rho) \cos \left(\phi(\tilde{\rho})+\sigma_{2}(\rho)\right)=(1+x) \exp \left\{\frac{y}{1+x}-\frac{1}{2} \frac{y^{2}}{(1+x+\theta y)}\right\}
$$

para algum $\theta \in(0,1)$.

A aplicaçăo da fórmula açima gera

$$
Z_{N}\left(\sigma_{2}\right)=\int d \mu_{\beta}(\phi) \prod_{\beta \in \mathcal{N}}(1+z(\rho) \cos \phi(\bar{\rho})) e^{-S(\phi, \phi)}
$$




$$
\exp \left\{\frac{z(\rho) \cos \phi(\bar{\rho})\left(\cos \sigma_{2}(\rho)-1\right)}{1+z(\rho) \cos \varphi(\rho)}-\frac{1}{2} \frac{y^{2}}{(1+x+\theta y)^{2}}\right\}
$$

onde

$$
S(\rho, \phi)=\frac{z(\rho) \sin \phi(\bar{\rho}) \sin \alpha_{2}(\rho)}{1+z(\rho) \cos \phi(\tilde{\rho})}
$$

Temos estes limites inferiores

$$
\frac{z(\rho) \cos \phi(\rho)\left(\cos \left(\sigma_{2}(\rho)\right)-1\right)}{1+z(\rho) \cos \phi(\rho)} \geq-\frac{z(\rho)}{2(1-z(\rho))}[\sigma(\rho)]_{2 \pi}^{2}
$$

e

$$
-\frac{y^{2}}{(1+x+\theta y)^{2}}>-(1+\pi) \frac{z^{2}(\rho)}{(1-4 z(\rho))^{2}}\left(\sigma_{2}(\rho)\right)_{2 \pi}^{2}
$$

onde fizemos a aproximação $1-\cos k \leq k^{2} / 2, \mathrm{e}$

$$
z_{1}(\rho)=\frac{z(\rho)}{1-z(\rho)} \quad z_{2}(\rho)=(1+\pi) \frac{z^{2}(\rho)}{(1-4 z(\rho))^{2}}
$$

Assim

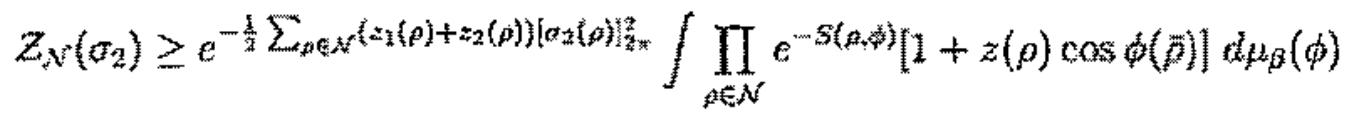

Como a funçảo $S(\rho, \phi)$ impar em $\phi$, a desigualdade de Jensen naquela variável dá

$$
Z_{\mathcal{N}}\left(\sigma_{2}\right) \geq \sum_{p \in \mathcal{N}} c_{N} e^{-[\max \phi s(p, \phi)]} \int \prod_{p \in \mathcal{N}}[1+z(p) \cos \phi(\bar{p})] d \mu_{p}(\phi)
$$

Uma yez que

$$
\left.S(p, \phi) \leq C \sum_{\rho \in \mathcal{N}} z(p)\left|\sigma_{2}(p)\right| \bmod 2 \pi\right]^{2}
$$

para alguma constant $\mathrm{C}$, contanto que $\beta$ seja tüo grande que $z(\rho)<1$, obtemos

$$
Z_{\mathcal{N}}\left(\sigma_{2}\right) \geq \mathrm{e}^{-\frac{1}{2} \sum_{\rho \in \mathcal{M}}\left\{\mu_{1}(\rho)+z_{2}(\rho)\right)\left[\sigma_{2}(\rho)\right]_{2 \pi}^{z}} \int \prod_{\rho \in \mathcal{N}}[1+z(\rho) \cos \phi(\bar{\rho})] d \mu_{\hat{\rho}}(\hat{\varphi})
$$

É possivel encontrar um limite superior para

$$
\left(z_{1}(\rho)+z_{2}(\rho)\right)\left[\sigma_{2}\right]_{2 \pi}^{2}
$$


usando linearidade de $\sigma_{2}(p)$ nas cargas e neutralidade de $p$.

Pela neutralidade, potemos denotamos por $p_{p}$ e $n_{t h}$ sítios contidos no suporte de $p$, tais que

$$
p_{z} \in\left\{(j: \rho(j)>0\}, \quad n_{z *} \in\{: p(j)>0\}\right.
$$

e escrevemos

$$
v_{2}(p)=\sum_{j \neq}\left[o_{2}\left(m_{n}\right)-\sigma_{2}\left(n_{n}\right)\right]
$$

onde a soma em $\mu$ contém $\frac{1}{2} \Sigma_{3}, p(j)$ termos. Lembrando que é sempre possivel representar uma densidade arbitrária de carga como o divergente de um carnpo vetorial es escrevemos

$$
\sigma_{2}\left(p_{15}\right)-\sigma_{2}\left(\eta_{k}\right)=\sum_{j}\left\{\epsilon_{1}(j) \partial_{1} \sigma_{2}(j)+\epsilon_{2}(j) \partial_{2} \sigma_{2}(j)\right\}
$$

onde $\epsilon_{k}=0, \pm 1$, sup $\epsilon_{k} \subset D(\rho)$, para $k=1,2$, com

$$
\left.\sum_{i}\left\|\epsilon_{1}(j)\right\|+\left\|\epsilon_{2}(j)\right\|\right\} \leq 2 d(p)
$$

Da definiça de de $\sigma_{2}(\rho)$ vem

$$
\xi^{-1}\left(\partial_{1} \sigma_{2}\right)\left(j_{1}, j_{2}\right)=\partial_{2}\left[C\left(j_{1}, j_{2}\right)-C\left(j_{1}-x, j_{2}\right)\right]
$$

e

$$
\xi^{-1}\left(\partial_{2} \sigma_{2}\right)\left(j_{1}, j_{2}\right)=-f^{*}\left(j_{1}, j_{2}\right)+\partial_{1}\left[C\left(j_{1}, j_{2}\right)-C\left(j_{1}-x, j_{2}\right)\right]
$$

Logo, temos um limite superior da forma

$$
\left(z_{1}(\rho)+z_{2}(\rho)\right)\left[\sigma_{2}\right]_{2 \pi}^{2} \leq z(d(\rho) \log d(\rho))^{2} \xi^{2}\left|\Delta \sigma_{2}\right|^{2}
$$

onde $d(\rho)$ é o diâmetro do suporte de $\rho$, o qual em nosso caso é $\left(4 / 3 L_{k}\right)^{2}$. Conforme as renormalizaçöes das atividades do gás, vimos que, para $\beta$ suficientemente grande, há sempre uma constante $t$ tal que

$$
z \leq \exp \left(-t \log L_{k}\right) \exp \left(-C_{\beta}|\rho|^{2}\right)
$$

Substituindo esses valores em (4.8) obtemos, para $|z|<1 / 4$,

$$
\begin{aligned}
& \left\langle D_{0 x}^{*}(\beta, \lambda)\right\rangle \leq e^{C( \pm)} e^{-(1 / 2) E_{x}^{(2-1)}} e^{-\beta^{-1} \xi^{2} \delta(C(0)-C(x)} \\
& \leq e^{-\left\{\hat{k}^{2} / 2 p\right) \log (1+|x|)}
\end{aligned}
$$

para $\delta<1,0$ que completa 0 argimento. 


\section{Capítulo 5}

\section{Rotores Quânticos}

Para esta apresentação procuramos ser o mais geral possivel quanto ta escoTha do sistema de rotores que escolheremos. O único requerimento imposto ao Hamiltoniano dos rotores é que seu potencial seja periódico. A análise do modelo tem duas partes. Primeiro, usamos uma representatăa de espaço de fase - por meio da fórmula de Trotter - para mapear o sistema quânitico unidimensional ern um sistema classico bidimensional. Como exemplo, escrevemos a funçăo de partição de rotores cujo Hamiltoniano tem parte cinética quadrática no momento angular e interação da forma co-senoidal.

En seguida, por meio de uma transformaçäo de dualidake aplicada ao sistema clássico, obtemos a função de partição do gás no espaço dual (A transformaçăo de dualidade é tema do próximo capítulo). Dessa representaçâo passamos à representação das cargas, na qual a função de partiçăo obtida $\dot{e}$ aguela de um gás interagente através de um potencial geral em duas dimensōes, dependente das funçôes que definem o Hamiltontano os rotores.

\subsection{A Função de Partição}

Seja $\Lambda$ um subconjunto finito de $Z$. A cada ponto $x \in Z$ está associada uma variável $\theta(x) \in[-\pi ; \pi]$, a coordenada angular do rotor. $\operatorname{Seja} a^{1} p(x)=-1 \frac{\partial}{\partial \theta(x)} \circ$ momento angular conjugado à variável $\theta(x)$. Vamos definir o Hamiltoniano de um sistema de $N$ rotores em termos de funçöes arbitrárias tanto para a parte cinética quanto para a parte da interação do Hamiltoniano. Assim,

\footnotetext{
in $=1$
} 
para esses rotores am $A=Z \cap[-N, N]$ definimos

$$
H=\sum_{x=-N}^{N} f(p(x))-\sum_{x=-N}^{N} V(\theta(x)-\theta(x+1))
$$

Periodicidade em $\theta$ é a única condiçăo que impomos à interaçấo. O opera dor $p(x)$ é tomado com condiçöes periódicas de contorno, de modo que seu espectro é discreto $\left\{k_{x}, k_{x} \in Z\right\}$, e satitsfaz as relaçồ de comutação:

$$
[p(x), b(y)]=-i \delta_{z z}
$$

$O$ sistema e tomado com condicoos pariólieas de contorno, $0(N+1)=\theta(-N)$. O Hamitoniano age no espaço the Hilbert $L^{2}\left([-\pi,+\pi]^{|\lambda|}\right)$, ou seja, o espaço de $N=|\Lambda|$ particulas movendo-se em circulo.

Lema 5.1 A função de partição do modelo de rotores quânticos em $\mathrm{d}=1$ dimensäo pode ser escrita como uma funçã̃o de particho de um gás clássico em $d=2$ dimensōes, cuja medida do estado de equilíbrio é dado por

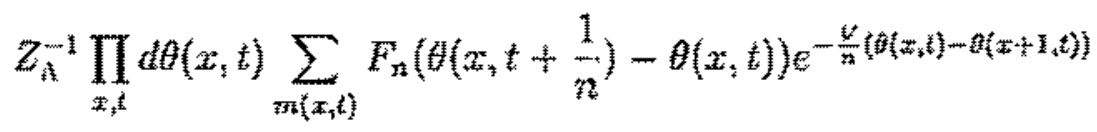

onde

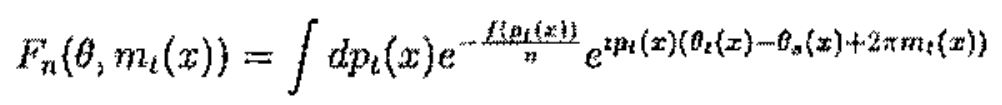

e $m_{t}(x)$ säo nümeros inteiros.

Para mostrar esse resultado, partumos da definição da funçäo de partição do nodelo:

$$
\mathcal{Z}=r \mathrm{r}\left[\exp -g\left(\mu_{0}+\nu\right)\right]
$$

onde usnmos a notaçăo

$$
H_{0}=\sum_{x=-N}^{N} f(p(x))
$$

e

$$
V=\sum_{x=-N}^{N} V(\theta(x)-\theta(x+1))
$$

Para calonlaz $(3.1)$ adotamos a base que hagonaliza $V$, que

$$
\mid \theta)=\prod_{x=-N}^{N}|\theta(x)\rangle
$$


e corn a qual podemos efetuar o cálculo explicito do traço acima:

$$
Z=\int_{[-;, \pi]}^{N} D \theta\left\langle\theta\left|\exp -\theta\left(H_{0}+\nu\right)\right| \theta\right\rangle
$$

onde

$$
D \theta=\prod_{x=1}^{N} \int_{\ldots ;}^{n} d \theta(x)
$$

Para reduzir o problema de tratar com operadores que nëo comutam a um problema semelhante ao problema clássico em uma dimensäo maior, fazemos uso da formula de Trotter.

Lema 5.2 Para operadores $A, B$ limitados inferiomente

$$
e^{A+n}=\lim _{n \rightarrow \infty}\left(e^{n / n} e^{k / n}\right)^{n}
$$

Prova. (Ver [10]) Escrevemos, entäo

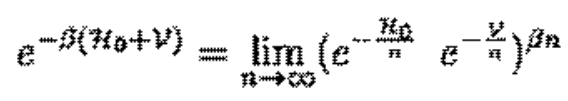

Vamos supor que in seja inteiro."

Ao efetuar o cálculo acima inserimos a decomposição da unidade

$$
\int_{-\pi}^{\pi} d \theta_{t}\left|b_{t}\right|\left\langle\theta_{t}\right|
$$

entre cada um tos $n \beta-1$ pares de fatores do produto de exponenciais que forma $e^{\text {ti }}$.

Chegamos à representação

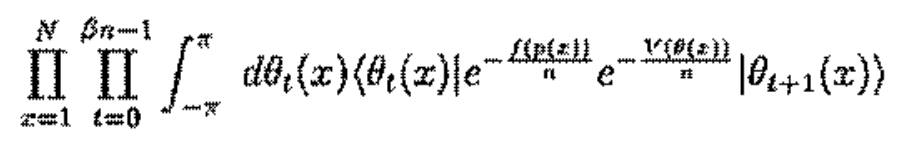

com a condiçăo de periodicidade $\theta_{0}=\theta_{\beta n}{ }^{3}$

Já que $\mathrm{V}$ é diagonal na base escolhida, temos

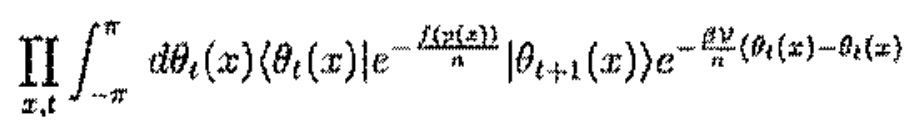

\footnotetext{
"Isso é so urna conveniência aqqui. Adiante faremos $\beta$ qualqquer.

${ }^{3}$ A partir daqui quando se escrever o produtório de $x$ e $t$ entenda-se produto em $-N \leq$ $x \leq N$, e $0 \leq t \leq \beta n-1$
} 
O cálculo de cada $\left\langle\theta_{t}(x)\left|\exp \left[-\frac{(p i x)}{n}\right]\right| p_{s}(x)\right)$ é idèntico àquele do valor esperado, na representaçāo das coordenadas, de uma funçăo do momento de uma partícula movendo-se em círculo. Efenamo-lo assim

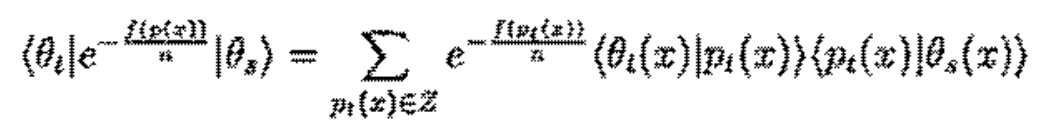

Substituindo o valor esperado

$$
\left\{\theta_{t}(x) \mid p_{t}(x)\right)=\frac{1}{\sqrt{2 \pi}} e^{z p_{t}(x) \theta_{t}(x)}
$$

vern

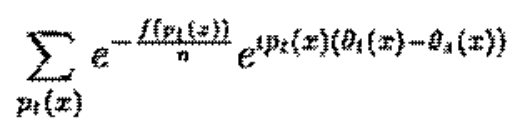

A fórmula de Poisson nos garante que ê licito integrar nas variảveis p, como se contínuas fossem, contanto que alteremos o argumento em $\theta$ do que está para

$$
\theta_{4}(x)-\theta_{s}(x)+2 \pi m_{l}(x)
$$

com $m_{t}(x)$ inteiro, e acrescentemos uma soma externa sobre $m_{t}(x)^{4}$ :

$$
\sum_{m_{t}(x) \in Z} \int d p_{t}(x) e^{-\frac{\left(p_{i}(x)\right)}{n}} e^{i p t(x)\left(p_{t}(x)-\theta_{n}(x)+2 \pi m_{t}(x)\right\}}
$$

Seja $F_{n}\left(\theta, m_{y}(x)\right)$ é a transformada de Fourier de da parte cinética do Hamiltoniano:

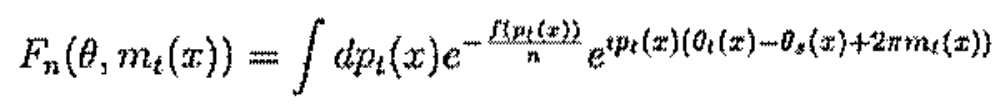

A funçäo de partição fica

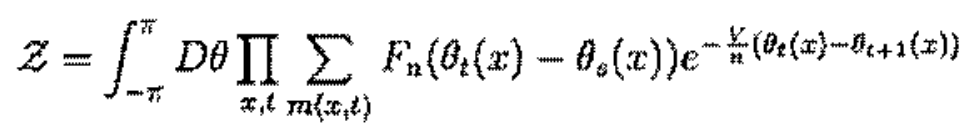

com condiços periódicas de contorno $\theta_{0}(x)=\theta_{B n}(x)$.

Uxn passo para a simplificą̧ão dessa fốrmula consiste em deserever cada

\footnotetext{
"Estamos elininamos conktantes destecessárous
} 
possivel conjunto de valores de $f_{t}(x)$ como um campo bidimensional, introduzindo uma nova variável na direção 'temporal'

$$
\theta_{t}(x) \rightarrow(x, 0)
$$

Simultaneamente, fazemos um reescalonamento da variavel $t$ da forma

$$
t \rightarrow \frac{t}{n}-\frac{p}{2}
$$

de modo a termos uma estrutura de teoria no continuo. Corresyondentemente temos

$$
\theta=\left\{\theta(x, b), x \in A, t \in \frac{Z}{n} \cap\left[-\frac{p}{2}, \frac{\beta}{2}\right)\right\}
$$

Assim se completa a correspondência entre o modelo quântico unidimensional s o modelo clássico bidimensional.

\subsection{Exemplos de Rotores Planos}

O potencial do rotor plano é descrito pela interaçäo

$$
\nu=J \sum_{x=-N}^{N} \cos (\theta(x) \cdots \theta(x+1))
$$

com acoplamento ferromagético, $J>0$.

Dois exemplos de formas admissiveis para o termo cinético são

1. O Módulo. Seja

$$
H_{0}=\sum_{x=-N i}^{N} \frac{|p(x)|}{2 I}
$$

onde I é o momento de inércia de cada rotor da rede.

A função de partição é dada por ${ }^{5}$

$$
Z=\int_{-\pi}^{\pi} \prod_{x, i} w_{i}\left(\theta(x, t)-\theta\left(x, t+\frac{1}{n}\right)\right) v_{t}(\theta(x, t)-\theta(x+1, t)) d \eta(x, t)
$$

\footnotetext{
${ }^{3}$ Kote que devido ao reescalonamento na vatútyel, o produtóto corre em $-N \leq x \leq N$ e $-\frac{\beta 3}{2} \leq t \leq \frac{a}{2}$.
} 
com

$u_{t}\left(\theta(x, t)-\theta\left(x, t+\frac{1}{n}\right)\right)-\sum_{m_{0}} \frac{-(1 / 1 n)}{(1 / 2 I n)^{2}+\left[\theta(x, t)-\partial\left(x_{1} t+\frac{1}{n}\right)+2 \pi m_{0}(x, t)\right]^{2}}$

e

$$
v_{J}(\theta(x, t)-\theta(x+1, t))=\exp \left\{-\frac{J}{n} \cos (\theta(x, t)-\theta(x+1, t))\right\}
$$

onde $m=\left\{m(x, b), x \in \Lambda_{*}, E \frac{z}{n} \cap\left[-\frac{2}{2}, \frac{j}{2}\right]\right\}$.

2 Fr, cés Qar aticas Seja

$$
H_{0}=\sum_{x=-N}^{N} \frac{p^{2}(x)}{2 Y}
$$

Nesse caso a função de partiçăo ainda toma a forma (5.1), com

$$
v_{1}\left(\theta(x, t)-\theta\left(x, t+\frac{1}{n}\right)\right)=\sum_{m_{0}} \exp \left\{-\frac{I n}{2 \beta}\left[\theta(x, t)-\theta\left(x, t+\frac{1}{n}\right)+2 \pi m_{0}(x, t)\right]^{2}\right\}
$$

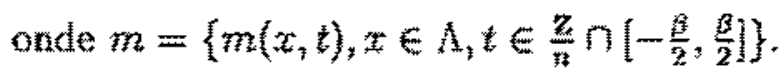

Ǩlein e Perez [33] têm resultados de localizaçāo do stado fundamental desses rotores com momantos de inércia aleatórios.

\subsection{A Aproximaçāo de Villain}

A interação de Villain k obtida pela substituição da forma de co-seno da interação por uma gaussiana periotizada. Note que na funģăo de partiçầo do rotor plano dada na seço anterior, a forma de Villain foi tomada na direçăo temporal. Neste ponto, gostarímos de adotar o potencial de Vllain também na direçăo espacial. Vilain determiniou as condiçoes para que a funçäo

$$
e^{z \cos \hat{\theta}}
$$

pudesse ser aproximada por

$$
\sum_{J n=Z} e^{-\gamma(z)(0+2 \vec{n} m)^{2}}
$$


onde, em nosso caso, $z=\frac{J}{n}$, e $\gamma(z)$ é uma constante a ser determinada. O resultado é a identificação [12], [19].

$$
\gamma(z)= \begin{cases}\frac{z}{2}\left(1-\frac{1}{2 z}\right) & \text { para } z \rightarrow \infty \\ \frac{1}{4 \log \frac{2}{z}} & \text { para } z \rightarrow 0\end{cases}
$$

Em nosso caso $z=J / n$ é pequeno. No entanto, vamos desconsiderar isso, e adotar a aproximaçāo para $z \gg 1$ assim mesmo. Em essência estamos mudando nosso sistema inicial. $\mathrm{O}$ efeito disso será trocar a dependência de $\gamma(z)$ de $1 / \log n$ para $1 / n$. Esta última forma é mais adequada para a realização de transformações de dualidade que faremos no Capítulo 6 Assim, em vez do co-seno, a nova interação será dada por

$$
\sum_{m=-\infty}^{\infty} e^{-(z / 2)\left(\theta_{i}-\theta_{j}+2 \pi m\right)^{2}}
$$

Repetindo o raciocínio para cada para da interação, vem

$$
\exp -\frac{\mathcal{V}(\theta)}{n}=\prod_{x} \sum_{m(x)=-\infty}^{\infty} \exp -\frac{J}{2 n}[\theta(x)-\theta(x+1)+2 \pi m(x)]^{2}
$$

com $m(x)$ inteiro.

Note que essa transformação preserva a simetria do problema, no sentido de que em baixas temperaturas os dois modelos tem o mesmo comportamento. O novo potencial possui a simetria abeliana global na variable $\theta$.

Nessa aproximação a função de partiçảo pode ser escrita como

$$
\mathcal{Z}=\int_{-\pi}^{\pi} \prod_{x, t} v_{f}\left(\theta(x, t)-\theta\left(x, t+\frac{1}{n}\right)\right) v_{J}(\theta(x, t)-\theta(x+1, t)) d \theta(x, t)
$$

com

$$
v_{I}\left(\theta(x, t)-\theta\left(x, t+\frac{1}{n}\right)=\sum_{m_{0}} \exp \left\{-\frac{I n}{2}\left[\theta(x, t)-\theta\left(x, t+\frac{1}{n}\right)+2 \pi m_{0}(x, t)\right]^{2}\right\}\right.
$$

$\mathrm{e}$

$$
v_{J}(\theta(x, t)-\theta(x+1, t))=\sum_{m} \exp \left\{-\frac{J}{2 n}[\theta(x, t)-\theta(x+1, t)+2 \pi m(x, t)]^{2}\right\}
$$

onde $m=\left\{m(x, t), x \in \Lambda, t \in \frac{\mathbf{Z}}{n} \cap\left\{-\frac{\beta}{2}, \frac{\beta}{2}\right]\right\}$. 
Observação . Há uma formulação alternativa do problema que consiste em preservar a temperatura inversa $\beta$ como parâmetro explícito na fórmula de Trotter. Seguindo esse caminho, em vez de (5.2), obtemos

$$
e^{-\beta\left(\mathcal{H}_{0}+\mathcal{V}\right)}=\lim _{n \rightarrow \infty}\left(e^{-\frac{\beta \mathcal{H}_{0}}{n}} e^{-\frac{\beta \mathcal{V}}{n}}\right)^{n}
$$

Com isso a funçāo de partiçāo (5.3) fica

$$
\mathcal{Z}=\int_{-\pi}^{\pi} D \theta \prod_{x, t} \sum_{m(x, t)} F_{n}\left(\theta_{t}(x)-\theta_{s}(x), m_{t}(x)\right) e^{-\frac{\partial V}{n}\left(\theta_{t}(x)-\theta_{t+1}(x)\right)}
$$

com

$$
F_{n}\left(\theta, m_{t}(x)\right)=\int d p_{t}(x) e^{-\frac{\beta f\left(p_{t}(x)\right)}{n}} e^{t p_{t}(x)\left(\theta_{t}(x)-\theta_{s}(x)+2 \pi m_{t}(x)\right)}
$$

onde a variável $t$ assume valores de 0 a $n$. Aqui também cabe introduzir uma nova variável na direção temporal pelo reescalonamento de $t$, agora da forma

$$
t \rightarrow \frac{\beta}{n} t-\frac{\beta}{2}
$$

A funçāo de partiçāo passa a ser dada por

$$
\mathcal{Z}=\int_{-\pi}^{\pi} \prod_{x, t} v_{I}\left(\theta(x, t)-\theta\left(x, t+\frac{\beta}{n}\right)\right) v_{J}(\theta(x, t)-\theta(x+1, t)) d \theta(x, t)
$$

com

$$
v_{I}\left(\theta(x, t)-\theta\left(x, t+\frac{\beta}{n}\right)=\sum_{m_{0}} \exp \left\{-\frac{I n}{2 \beta}\left[\theta(x, t)-\theta\left(x, t+\frac{\beta}{n}\right)+2 \pi m_{0}(x, t)\right]^{2}\right\}\right.
$$

e

$$
v_{J}(\theta(x, t)-\theta(x+1, t))=\sum_{m} \exp \left\{-\frac{J \beta}{2 n}[\theta(x, t)-\theta(x+1, t)+2 \pi m(x, t)]^{2}\right\}
$$

Correspondentemente temos

$$
\theta=\left\{\theta(x, t), x \in \Lambda, t \in \frac{\beta \mathrm{Z}}{n} \cap\left[-\frac{\beta}{2}, \frac{\beta}{2}\right]\right\}
$$

De agora em diante vamos trabalhar nessa rede de espaçamento unitário na direçāo $x$, e espaçamento $\delta=\beta / n$ na direção temporal. 


\subsection{A Estimativa de McBryan-Spencer}

Essa estimativa é uma garantia de que não há transição de primeira ordem, embora nada se possa afirmar acerca de transicões de ordens mais altas. Os ingredientes da prova sāo periodicidade e analiticidade. Nosso resultado é de mesma natureza daquele obtido em [33], exceto que aqueles autores consideraram a forma do co-seno do potencial, sem aproximações . No entanto a translação complexa por eles efetuada é função do tempo apenas. Em nosso caso, podemos realizar uma translaçāo arbitrária.

Teorema 5.3 Seja

$$
G_{\Lambda, \beta}^{(n)}\left((y, s),\left(y^{\prime}, s^{\prime}\right)\right)=\left\langle e^{\mathrm{z}\left(\theta(y, s)-\theta\left(y^{\prime}, s^{\prime}\right)\right.}\right\rangle_{\lambda, \beta}^{(n)}
$$

onde $\langle\cdot\rangle_{\Lambda, \beta}^{(n)}$ é o valor esperado com relaçāo à medida dada em (5.8).

Seja $G\left((y, s),\left(y^{\prime}, s^{\prime}\right)\right)$ o limite de $G_{\Lambda, \beta}^{(n)}\left((y, s),\left(y^{\prime}, s^{\prime}\right)\right)$ quando $\Lambda, \beta, n \rightarrow \infty$.

Entāo

$$
G\left((y, s),\left(y^{\prime}, s^{\prime}\right)\right) \leq e^{-\sqrt{\frac{I J}{4 \pi^{2}}} \ln \sqrt{\left(y-y^{\prime}\right)^{2}+(J / I)\left(s-s^{\prime}\right)^{2}}}
$$

Prova. Seja $a(x, t)$ uma função quadraticamente integrável. Fazemos a translação complexa [33]

$$
\theta(x, t) \rightarrow \theta(x, t)+\imath a(x, t)
$$

nas variáveis de integração na expressão da função de correlação. Aqui $a$ é real e será escolhido logo adiante. Substituindo a translação acima na função de correlaçāo, obtemos a estimativa

$$
\begin{array}{r}
\left\langle e^{z\left(O(y, s)-O\left\langle y^{\prime}, s^{\prime}\right)\right.}\right\rangle \leq e^{-\left(a(y, s)-a\left(y^{\prime}, s^{\prime}\right)\right.} e^{-\frac{\ln }{2 \phi} \sum_{x, t}(a(x, t)-a(x, t+\beta / n))^{2}} \\
e^{\frac{J \beta}{2 n} \sum_{x, t}(a(x+1, t)-a(x, t))^{2}}
\end{array}
$$

onde os termos complexos foram estimados pelo módulo, $\left|e^{i x}\right|=1$, e a função de partição no denominador foi cancelada.

O lado direito de (5.9) pode ser escrito como forma quadrática

$$
\left\langle e^{z\left(\theta(y, s)-\theta\left(y^{\prime}, s^{\prime}\right)\right.}\right\rangle \leq e^{-\left(a(y, s)-a\left(y^{\prime}, s^{\prime}\right)\right)} e^{-\frac{\beta}{2 n}(a,-\Delta a)}
$$


onde definimos o produto escalar por

$$
(f, n)=\sum_{x, \imath} f(x, t) g(x, t)
$$

e é o Laplaciano de diferenga finta em duas dimensões, dado pot

$$
-\Delta=\sqrt{ } \partial_{1}^{*} \partial_{1}+I \partial_{2}^{*} \partial_{2}
$$

onde $\partial^{*}$ e operador adjunto definido en $(2.5)$

$$
a_{1}(x, t)=f(x+1, t)-f(x, b)
$$

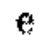

$$
\partial_{2} f\left(x_{1} t\right)=\frac{f\left(x_{2} t+\delta\right)-f\left(x_{2} t\right)}{\delta}
$$

Desse modo temos que

$$
\begin{aligned}
-\Delta f(x, t) & =2 f(x, y)-f(x+1, t)-f(x-1, t) \\
& +\delta^{-2}(2 f(x, t)-f(x, t+\delta)-f(x, t-\delta)
\end{aligned}
$$

Escolhemos

$$
a(x, t)=C(x-y, t-s)-C(x-y, t-s)
$$

onde $C(x, t)$ é a funçăo de Green do operador $A$, dada por

$$
C(x-y, t-s)=\frac{1}{2 N+1} \frac{1}{\beta} \sum_{p q q} \frac{\exp (\imath p(x-y)+q(t-s))}{I^{-1}(2-2 \cos p)+J^{-1} \delta^{-2}(2-2 \cos q \delta)}
$$

com

$$
p=\frac{2 \pi}{2 N+1} m_{1}, \quad-N \leq m_{1} \leq N
$$

e

$$
q=\frac{2 \pi}{p} m_{2}, \quad-\frac{n}{2} \leq m_{2} \leq \frac{n}{2}
$$

Então

$$
\left\langle a_{3}-\Delta a\right\rangle=a(n)-a\left(y^{\prime} s^{\prime}\right)
$$


Obtemos

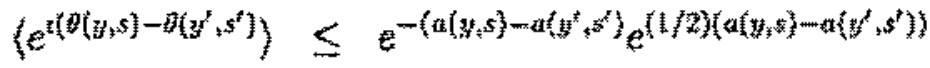

$$
\begin{aligned}
& \leq e^{-\{1 / 2)\left(a(y, s)-a\left(y^{2}, x^{2}\right)\right\}}
\end{aligned}
$$

Agora

$$
a(y, s)-a\left(y^{\prime}, s^{t}\right)=2\left(C(0,0)-C\left(y-y^{\prime} ; s-s^{\prime}\right)\right)
$$

$O$ comportanento assintótico da diferença $C(0,0)-C\left(y-y^{\prime}: s-s^{\prime}\right)$ quando $n \rightarrow \infty$ é calculado no Apêndice B para o caso especial em que $s-s^{t}=0$. O resultado geral, no entanto, pode ser facilmente deduzido da exposiçẵo que damos lá A conclusão é que para $\left|y-y^{*}\right|_{1}\left|s-s^{*}\right|$ suficienternente grandes $C(0,0)-C\left(y-y^{f}, s-s^{t}\right)$, é bern aproximado pelo propagador escalar contínuo sern massa em dutas dimensões [20]. Logo,

$$
\begin{aligned}
& \left(e^{s t\left(Q\{y, s\}-O\left\{y^{f}, s^{2}\right)\right.}\right) \leq e^{2\left(C\left(y-y^{f}, s-s^{f}\right\}-C(0,0)\right)} \\
& \leq e^{-\sqrt{\frac{13}{\pi^{2}}} \ln \sqrt{\left(y-y^{\prime}\right)^{2}+(J / I)\left[s-s^{\prime}\right)^{2}}}
\end{aligned}
$$




\section{Capítulo 6}

\section{A Transformação de Dualidade}

Por transformaçăo de dualidade entendemos o estudo de modelos duais ao Villain, os quais são obtidos por transformaçôes de Fourier nas variáveis angulares da funçầo de partiçảo (5.6):

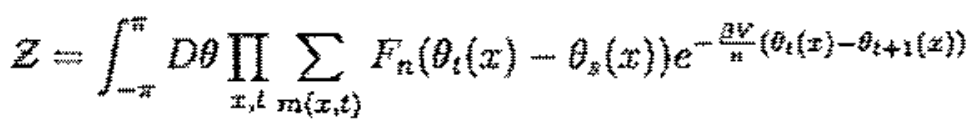

onde $F_{n}(\theta)$ é dado em $(5.7)$, e $V$ é ainda da forma co-seno.

A rede dual é conatruída pelo deslocamento dos sítios da rede original por metade do espaçamento de rede em todas as direşōes simultaneamente.

Consideramos o modelo em uma caixa retangular $\Lambda$ com condiçōes livres de contorno em $\partial \Lambda$. A cada par de vizinhos mais próximos $\left\{(x, t)\left(x^{\prime}, t^{t}\right)\right\}$ associamos funçồ com período $2 \pi$.

\subsection{Transformada de Fourier}

Partimos da funçäo de partição como obtida no capitulo anterior, e representamos tanto $F_{n}(\theta)$ quanto o potencial pelas respectivas transformadas de Fourier.

Os coeficientes da trausformada de Fourier de $F_{n}(\theta)$ têm a forma original da parte cinética da interaçăo, a única alteraçăo ế que a variável agora näo é mais $p$, mas um bond (pares de vizinhos mais próximos) $k_{x, t}$ :

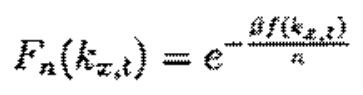


Já para o potencial admitiremos a seguinte expansāo.

$$
\left(e^{-\beta V^{\prime}(\theta)}\right)^{1 / n}=\sum_{l_{x, t} \in Z} g_{n}\left(l_{x, t}\right) e^{u_{x, t}(0(x, t)-\dot{O}(x+1, t))}
$$

$k_{x, t}$ e $l_{x, \mathrm{t}}$ são variáveis orientadas de bond direcionados de $(\mathrm{x}, \mathrm{t})$ a $(\mathrm{x}, \mathrm{t}+1)$, e de $(x, t)$ a $(x+1, t)$ respectivamente. ${ }^{1}$ Em teoria de gauge na rede, as variáveis fundamentais sāo definidas sobre bonds.

Substituimos a expressão acima na funçāo de partição (5.6), resulta

$$
\mathcal{Z}_{\Lambda}=\int_{-\pi}^{\pi} d \Theta \prod_{x, l} \sum_{k_{x, t}, l_{x, t}} e^{-\frac{\theta f\left(k_{x, t}\right)}{n}} g_{n}\left(l_{x, t}\right) e^{\left\{\left[k_{x, l}(\theta(x, t)-\theta(x+1, t))+l_{x, t}(\theta(x, t)-\theta(x, t+1))\right]\right.}
$$

A integral em $\theta$ gera funções deltas:

$$
\int_{-\pi}^{\pi} \prod_{x, t} d \theta(x, t) e^{\imath \theta(x, t)\left(\partial_{x} k_{x, t}+\partial_{t} l_{x, t}\right)}=\prod_{x, t} \delta\left(\partial_{x} k+\partial_{t} l\right)
$$

Agora, estabelecemos uma correspondência entre as variáveis de bond e variáveis inteiras definidas no centro de uma curva fechada formada por quatro bonds (plaquetas). Isso vai nos permitir eliminar a restrição da soma sobre valores das variáveis de bond que aparece devido à condição de divergência zero.

Usamos a versāo na rede do lema de Poincaré

$$
* \partial * u=0 \rightarrow u=* \partial \phi
$$

onde u é um campo vetorial $(1-$ form $)$ na rede, e $\phi$ é um $(\nu-2)-$ form, ou um campo escalar em $\nu=2$.

Temos

$$
\partial u=0, u=\partial m
$$

com $m$ um 2-form. Então, fazendo $m=* \phi$,

$$
u=\partial m=\partial * \phi=* \partial \phi
$$

Estabelecemos a correspondência nesse novo conjunto de variáveis definidas no centro dos sítios da rede dual $\Lambda^{*}=\Lambda+(1 / 2,1 / 2)$

$$
\begin{aligned}
l_{x^{*}, t^{*}} & =\phi\left(x^{*}, t^{*}\right)-\phi\left(x^{*}+1, t^{*}\right) \\
k_{x^{*}, t^{*}} & =\phi\left(x^{*}, t^{*}\right)-\phi\left(x^{*}, t^{*}+1\right)
\end{aligned}
$$

\footnotetext{
${ }^{1}$ Para evitar confusāo e salientar o carácter de variável de bond, deveríamos escrever $k_{(x, t)(x, t+1)}$ e $l_{(x, t)(x+1, t)}$ A notaçāo ficaria ruim, no entanto.
} 
As restrições são satisfeitas automaticamente nessas novas variáveis e, substituindo as expressöes (6.3) na função de partição acima, obtemos ${ }^{2}$

$$
Z_{\Lambda}=\sum_{\phi \in Z^{L+n}} \prod_{x, t} d \phi(x, t) e^{-\frac{\partial f\left(\partial_{x} \phi\right)}{n}} g_{n}\left(\partial_{t} \phi\right)
$$

onde a soma irrestrita sobre as variáveis da rede dual. ${ }^{3}$

Para escrever a iunção de partição na forma integral, impomos a condição de integrabilidade em $\phi$ através de funcōes deltas:

$$
Z_{n}=\int \prod_{x, l}\left(\sum_{n\{t, k)} \delta(\phi(x, t)-m(x, t))\right) d u_{f, g}(\phi)
$$

onde

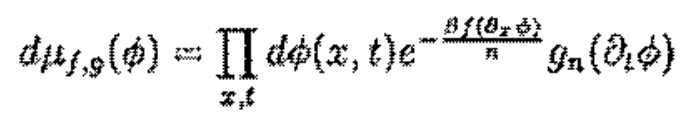

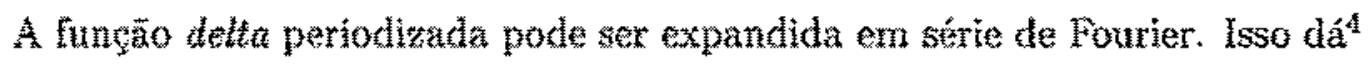

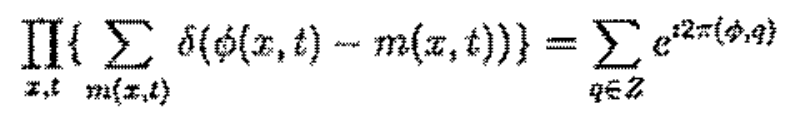

onde

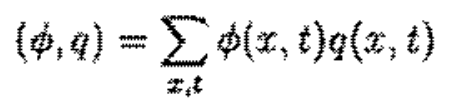

De forma cue

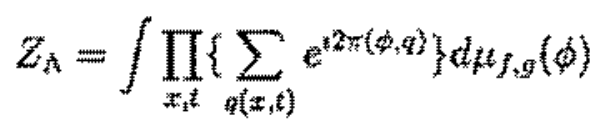

on

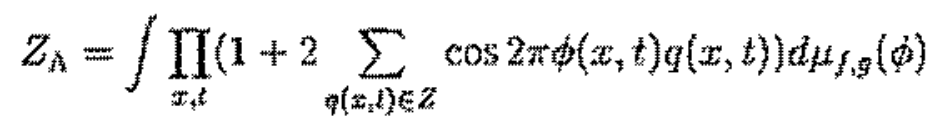

\footnotetext{
"Yoltamos a usar $x$ para representar os sitios da rede dual.

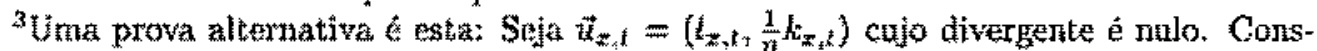
truinos, a partir de $\vec{t}_{x, t}$, o vetor $\tilde{y}_{x, t}=\left(-\frac{1}{t} k_{x, t}, l_{x, t}\right)$, cujo rotacional é zero. $\dot{\mathrm{E}}$ sempre possivel escolher como o gradiente de um campo escalar $M_{2}$ de modo que $l_{x ; t}=n(M(x, t)-M(x+1, t)) \cdot k_{x, t}=n(M(x, t)-M(x, t+1))$. Como $M \in \frac{\phi}{n}$, com $\phi \in Z$, temos $(6.4)$ de novo.

"Quando $d \mu_{v, f}$ êma medida gaussiana com média fe covariuncia $y$ a substituição da série de fourier pela soma sobre m na funçăo de partiçăo, perfazendo a integração em $\phi$, dá a representaçăo de Blanks do gás de Coulonb [14]: $Z=\sum_{q \in Z} e^{e^{t(q . J)}} e^{-\frac{1}{2}(q, v q)}$.
} 
Essa fórmula é uma prescrição para obter a função de partição dado o Hamiltoniano. A parte cinética se reproduz na função de partição - apenas seu argumento muda de $p(x)$ para $\partial_{x}(x, t)$. Quanto ao potencial, toma-se a sua transformada de Fourier - alterando-lhe o argumento de $\theta$ para $\partial_{t}(x, t)$.

$O$ potencial original é de fato uma função da derivada espacial de $\theta$ na rede de espaçamento unitário. Após a transformação de dualidade essa função passou a depender da derivada da nova variável na direção extra que surgiu como decorrência da aplicaçāo da fórmula de Trotter. Isso ocorre porque a transformaçāo de dualidade troca bonds horizontais e verticais.

\subsection{O Modelo Dual de Villain e a Represen- tação das Cargas}

Vamos nos restringir ao segundo modelo dado na seção 5.2 - cuja parte cinética sāo funçōes quadráticas do momento angular --, com a aproximação de Villain para o potencial. Para esse modelo, a função de partição é dada em (5.8). A transformaçāo de dualidade que leva a (6.8) fornece aqui

$$
Z_{\Lambda}(x)=\int \prod_{x, t}\left\{\sum_{\{q(x, l)\}} e^{22 \pi(\phi, q)}\right\} e^{-\frac{\delta}{2}(\phi,-\Delta \phi)}
$$

onde o índice $v$ indica a medida de Villain

$$
d \mu_{v}(\phi)=e^{\frac{-\delta}{2} \sum_{x, t}\left\{\frac{2}{T}(\phi(x, t)-\phi(x+1, t))^{2}+\frac{1}{J}\left(\frac{\phi(x, t)-\phi(x, t+\beta / n)}{\beta / n}\right)^{2}\right\}} D \phi
$$

$\operatorname{com} D \phi=\prod_{x, t} d \phi(x, t)$.

Estamos usando a notaçāo de produto escalar da seção 5.4, com laplaciano dado por

$$
-\Delta=\frac{1}{I} \partial_{1}^{*} \partial_{1}+\frac{1}{J} \partial_{2}^{*} \partial_{2}
$$

Efetuando a integral Gaussiana (6.10), obtemos a função de partição na representação das cargas. Usamos a identidade

$$
\int e^{z(\phi, q)} d \mu_{v}(\phi)=\int e^{-\frac{1}{2}\left(q,(-\Delta)^{-1} q\right)} \prod_{x, t} d \lambda(q(x, t))
$$


onde

$$
d \lambda(q)=\sum_{m \in} \delta(q-2 \pi m) d q
$$

Na representaçäo das cargas a funçẫo de particẫo ê

$$
\mathcal{Z}=\int e^{-\frac{1}{2}\left(a,-\Delta^{-1} q\right)} \prod_{x, t} d \lambda(g(x, t))
$$

O problema está entăo mapeado em um problema de cargas interagentes corn neutralidade elétrical geral- através de um potencial de Coulomb.

\subsection{Estimativa da Correlação entre Cargas Externas}

O resultado desta seção é de mesma natureza daquele apresentado sem provas na seção 3.3 para a correlaçäo de gases clássicos, mas claro que levando em conta os limites a serem tomados.

Dado o gás de Villain cuja funçẫo de partiçăo é (6.13), colocamos no sistema duas cargas fracionárias, uma $+\xi$, situada na origem, outra, $-\xi$, situada en 2. Na presença dessas cargas externas a funçăo de partição passa a ser

$$
\mathcal{Z}^{\xi}(x)=\int \mathrm{e}^{-(1) / 2)\left(q_{\mathrm{N}}+p(y, t),-\Delta^{-1}\left(\gamma_{\alpha}+p(y, t)\right)\right.} \prod_{y, i} d \lambda q(y, t)
$$

onde

$$
\rho(y, t)=\xi\left(\partial_{y 0}-\delta_{y z}\right) \delta_{40}
$$

A função tle correlação ê dada por

$$
G_{A}^{k}(x)=Z^{-1} \int e^{-(1 / 2) E\left(g_{A}+p(y, k)\right)} \prod_{y, k \in A} d \lambda(q(y, t))
$$

onde

$$
E\left(q_{\mathrm{A}}+\rho(y, t)\right)=(1 / 2)\left(q_{\mathrm{A}}+\rho(y, t),-\Delta^{-1}\left(q_{\mathrm{A}}+\rho(y, t)\right)\right)
$$

Podemos escrever a função de correlação como

$$
G_{A}^{*}(x)=\int e^{-(1 / 2)\left[E\left(q_{A}+p(z, t)\right]-E\left(t_{k}\right)\right.} d \lambda^{*}\left(a_{A}\right)
$$


onde

$$
d \lambda^{\prime}\left(q_{\Lambda}\right)=\mathcal{Z}^{-1} e^{-(1 / 2) E\left(q_{N}\right)} \prod_{y_{i} \in \in} d \lambda(q(y, t))
$$

Aplicamos a desigualdade de Jensen na variável $q$ para obter

$$
G_{A}(x) \geq e^{-(1 / 2) \int\left[E\left\{q_{\Lambda}+p(y, t)\right)-E\left(q_{\Lambda}\right)\right] d \lambda^{2}\left(q_{\Lambda}\right)}
$$

Expandindo o expoente $E\left(q_{A}+\rho(t, t)\right)-E\left(q_{A}\right)$, obtemos

$$
G_{A}^{t}(x) \geq e^{-(1)} \int d \lambda^{t}\left(q_{A}\right)\left\{\left(q_{A}(-\Delta)^{-1} p(t, t)\right]+\left(p(y, t),(-\Delta)^{-1} p(y, t)\right)\right]
$$

Como a nova medida $d \lambda^{\prime}\left(q_{N}\right)$ é simátrica e normalizada, obtemos simplesmente

$$
G_{A}^{\xi}(x) \geq e^{-(1 / 2)\left(p(y, t),(-\Delta)^{-1} p(s, t)\right)}
$$

Ternos

$$
\left(p(y, t),(-\Delta)^{-1} p(y, t)\right)=2 z^{2}(C(0,0)-C(x, 0))
$$

No Apêndice B calculamos exatamente a diferença entre as funçöes de Green acima, obtendo

$$
C(0,0)-C(x, 0) \sim \sqrt{\frac{I J}{4 \pi^{2}}} \ln |x|
$$

Então

$$
G_{\Lambda}^{\xi}(x) \geq C_{\xi} e^{-\xi^{2} \sqrt{\frac{l J}{t \pi^{2}}} \ln |x|}
$$

$\operatorname{com} 0<C_{\xi}<\infty .2$

\subsection{O Operador de Desordem}

Na seção 6.1 a transformaçà̃o de dualidade foi aplicada à funçăo de partiçăo da forma

$$
f(\theta)=F_{n}(\theta) e^{-\frac{V(\theta)}{i}}
$$

onde $F_{n}(\theta)$ e dada por $(5.7)$, e $V(\theta)$ foi definida implicitamente por (6.2). Passamos, entäo, a representação da funcao de partição no espaço dual (6.5). Note que é também possivel obter a função de correlaçấo no espaço dual realizando a transformaçà̃o de dualidade a uma função convenientemento 
escolhida.

Seja $\omega$ uma trajecótia qualquer de $0 \mathrm{a} \times$. Se definimos a função

$$
f_{x, y}(\theta)=f(\theta) \exp \left[-\imath \chi_{x y} \xi \theta\right]
$$

onde $\chi_{x y}=1$ se $(x, y)$ é um par de vizinhos mais próximos contidos em $w$, arientado de acordo com $\omega_{1}$ e $\chi_{x y}=0$, caso contrário, e a ela aplicarmos a transformaçâo de dualidade, vamos obter a funçāo de correlaçäo $\left\langle e^{t \xi\left(\theta_{b}-\theta_{x}\right)}\right\rangle$ na representaçâo de sine-Gordon.

A transformaçäo de dualidade nos leva às variáveis duais correspondentes a $(6.4)^{*}$

$$
l_{x, 2}+\xi \chi_{x y}=\phi(x, 1)-\phi(x+1, t)
$$

e

$$
k_{x, i}+\xi \chi_{x y}=\phi(x, t)-\phi(x, t+1)
$$

A funçâo de correlaçăo $(5,9)$ entre os pontos $(y, s)=(0,0)$ e $\left(y^{\prime}, s^{\prime}\right)=(N, 0)$ no espaço dual ê entăo dada por

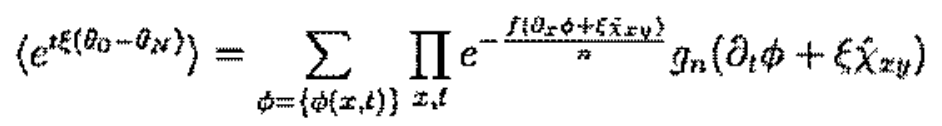

onde $\hat{\chi}_{x y}=1$. quardo $(x, y)$ é um par ordenado de vizinhos mais próximos em $\Lambda^{*}$, dual ao um bond na trajetória $\omega$, e $\hat{\chi}_{x, y}=0$ nos outros cassos.

Tomando a trajetória $\omega$ entre os pontos 0 e $N$ ao longo do eiso $x$, o valor esperado da correlaçäo para o modelo de Villain pode ser posto em função do valor esperado do operador de desordem:

$$
\left\langle e^{r\left(\theta_{0}-\hat{a}_{N}\right)}\right\rangle=\left\langle D_{0 N}^{E}\right\rangle
$$

onde $D_{b N}^{k}$ e o operador de desordem, aqui dado por

$$
\hat{D}_{N}^{\xi}(\phi)=\frac{v\left(\partial_{2} \phi+\xi\right)}{v\left(\partial_{2} \phi\right)}
$$

com

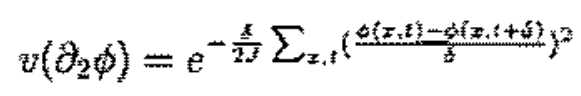

\footnotetext{
Dowxamos te usar o asteristo para indicar as variáveis do espaço dual.
} 
e $\xi(x, t)=\xi f^{N}(x, t)$ onde $f^{N}$ é definida por

$$
f^{N}(x, t)= \begin{cases}1, & \text { if } 1 \leq x \leq N, t=0 \\ 0, & \text { otherwise }\end{cases}
$$

de maneira análoga a (2.4), exceto que agora estamos levando en conta o espaçamento $\delta$ na direção temporal.

Deservolvendo (6.15), temos

$$
\hat{D}_{0 N}^{\xi}(\phi)=e^{-\frac{\delta}{2} \xi^{z} N} e^{-\frac{\xi}{J} \sum_{z=1}^{N}\{\phi(x, 0)-\phi(x,-\delta)\}}
$$

O valor esperado do operador de desordem é

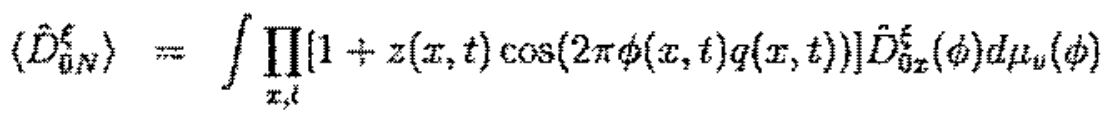

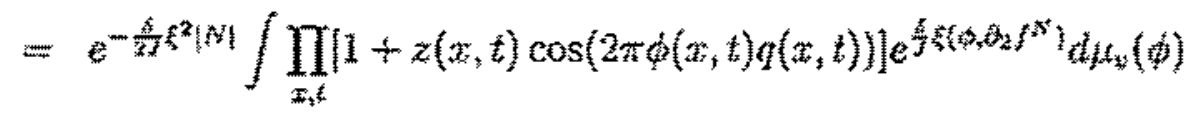

onde usamos que

$$
\left(\phi, \partial_{2} f^{N}\right)=\sum_{x=1}^{N} \frac{\phi(x,-\delta)-\phi(x, 0)}{\delta}
$$

Efetuamos a translaçâo rẹal $\phi \rightarrow \phi+\sigma$, onde $\sigma(x, t)=\frac{\varepsilon}{d}\left(-\Delta^{-1}\right) \partial_{2} f^{N}(x, t)$. $O$ motivo dessa escolha val ficar obvio adiante.

Com a mudança de variável, a medida gaussiana muda para

$$
d \mu(\phi+\sigma)=d \mu_{v}(\phi) e^{-\delta(\phi,-\Delta \sigma)-\frac{\xi_{2}}{2}(\sigma,-\Delta \sigma)},
$$

enquanto que

$$
\left(\phi+\sigma, \partial_{2} f^{N}\right)=\left(\phi, \partial_{2} f^{N}\right)\left(\sigma, \partial_{2} f^{N}\right)
$$

Corn a translação escolhida, o termo $-\left(\phi_{2}-\Delta \sigma\right)$ da medida cancelasse a exponencial de $\phi$ no integrando do valor esperado:

$$
-\left(\phi_{2}-\Delta \sigma\right)+\frac{\xi}{J}\left(\phi, \partial_{2} f^{N}\right)=0
$$

Quanto ao terno que sobra na medida gaussiana:

$$
-\frac{\delta}{2}\left(\sigma_{1}-\Delta \sigma\right)=-\frac{\delta \xi}{2 j}\left(\sigma, \partial_{2} f^{N}\right)
$$


Combinado com o fator da exponencial obtemos

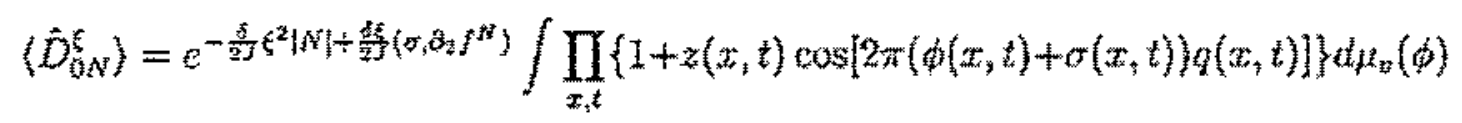

Mas

$$
\begin{aligned}
\frac{\delta \xi}{2 J}\left(\sigma, \partial_{2} f^{N}\right) & =\frac{\delta E}{2 J} \sum_{x, t} \sigma(x, b)\left(\partial_{2} f^{N}\right)(x, b) \\
& =\frac{\delta \xi}{2 J} \sum_{x=1}^{N} \frac{\partial(x,-n)-\sigma(x, 0)}{\delta}
\end{aligned}
$$

Temos

$$
\begin{aligned}
\sigma(x, t) & =\frac{\xi}{J} \sum_{y, s} C(x-y, t-s)\left(\partial_{2} f^{N}\right)(y, s) \\
& =\frac{\xi}{J} \frac{1}{\delta} \sum_{y=1}^{N}(C(x-y, t+\delta)-C(x-y, t))
\end{aligned}
$$

Logo,

$$
\sigma(x,-\delta)-\sigma(x, 0)=\frac{\xi}{J} \frac{1}{\delta} \sum_{y=1}^{N}(2 C(x-y, 0)-C(x-y, \delta)-C(x-y,-\delta))
$$

Substituindo a diferença acima em (6.17), obtemos

$$
\frac{\delta \xi}{2 J}\left(\sigma, \partial_{2} f^{N}\right)=\frac{\delta \xi^{2}}{2 J^{2}} \sum_{x, y=1}^{N} \frac{1}{\delta^{2}}\left(2 C\left(x-y_{1} 0\right)-C\left(x-y_{;}-\delta\right)-C(x-y, \delta)\right)
$$

Usando que (6.11), podemos escrever

$$
\begin{aligned}
\frac{\delta \xi}{2 J}\left(\sigma, \partial_{2} f^{N}\right) & =\frac{\delta \xi^{2}}{2 J^{2}}\{J N \\
& \left.+\frac{J}{I} \sum_{x, y=1}^{N}(C(x-y+1,0)-2 C(x-y, 0)+C(x-y-1,0))\right\}
\end{aligned}
$$

A soma acima se reduz à diferença entre os dois pontos $x-1, N$ :

$$
\frac{\delta \xi}{2 J}\left(\sigma, \partial_{2} f^{N}\right)=\frac{\delta \xi^{2}}{2 J^{2}}\left\{J N-2 \frac{J}{I}(C(0,0)-C(N, 0))\right\}
$$


Finalmente,

$$
\left\langle\hat{D}_{0 N}^{\xi}\right\rangle=e^{G(N)} Z(\sigma)
$$

onde $G(N)$ é a contribuição de ondas de spin ao valor esperado do operador de desordem:

$$
G(N)=-\frac{\delta \xi^{2}}{I J}(C(0,0)-C(N, 0))
$$

$\mathrm{e}$

$$
Z(\sigma)=\int \prod_{x_{1}, l}[1+z(x, t) \cos (2 \pi(\phi(x, t)+\sigma(x, t)) q(x, t))] d \mu_{v}(\phi)
$$

\subsubsection{Comentários}

Partindo da fórmula (6.18), gostaríamos de derivar uma expressāo para a função de partição como combinação convexa de estados que descrevem gáses diluídos de multipolos neutros, com a qual poderíamos estimar o valor esperado do operador de desordem. Como vimos no Capítulo 4, cada passo do processo consiste em construir ensembles esparsos, o que significa diminuir a densidade do gás. Sabemos que uma alteração na densidade provoca uma mudança na atividade do gás. No caso, estaremos aumentando a atividade. Esse é o custo entrópico do processo. Por outro lado, para que a medida de probabilidade na variável $\phi$ seja positiva, a atividade do gás não poderá ser maior do que 1. Logo o custo entrópico é compensado pela extração da auto-energia das cargas de cada ensemble, a qual tem de superar o aumento de entropia resultante do agrupamento de cargas em blocos esparsos.

É justamente aqui que o processo falha para o gás de Villain obtido acima. Uma translação complexa $\phi \rightarrow \phi+\imath a$ na variável de integração em $Z(\sigma)$ altera a medida para

$$
d \mu_{v}(\phi+\imath a)=d \mu_{v}(\phi) e^{-2 \frac{g}{n}(\phi,-\Delta a)} e^{-\frac{\delta}{2}\left(a_{1}-\Delta a\right)}
$$

e a exponencial para

$$
e^{2(\phi+i, q)}=e^{t(\phi, q)-(\alpha, q)}
$$

Assim

$$
Z(\sigma)=e^{-E(a)} \int \prod_{x, t}[1+z(x, t) \cos (2 \pi q(x, t)(\phi(x, t)+\sigma(x, t)))] d \mu_{v}(\phi)
$$


onde

$$
E(a)=-(a, a)+\frac{p}{2 a n}\|\Delta\|(a, a)
$$

A maximizaçăo de E(a) en relação a a leva ao valor buimo desse parámetro

$$
a=\frac{n}{\rho\|\Delta\|} \|^{n}
$$

para o gual

$$
E(a)=\frac{\beta J}{2 n}(a, a)
$$

onde fizemos $\|\Delta\|=\frac{1}{4}\left(\frac{n}{1}\right)^{2}$.

No caso gaussiano o fator de decaimento é exatamente a funçăo de Green do laplaciano calculada em $(0,0)$. A dependência mm $n$ é

$$
C(0,0)=\int_{-r+\beta / \beta}^{n+\beta / \beta} \frac{1}{p^{2}+q^{2}} \approx \frac{\beta}{n}
$$

Esse fator não gera nentum decaimento.

Jấ o custo entrópico de formaçäo de blocos cresce com n. Isso provém do cálculo do número de pontos em um bloco de lado $L$ em um plano cujo espaçamento é unitário na direçäo horizontal e vale $\beta / n$ na direçäo vertical. O número de pontos no interior do bloco é simplesmente

$$
\frac{n}{\beta} L^{2}
$$

Logo, para que o método empregado no capítulo 4 seja funcional, é necessário que a competição energiamentropia se realize favoravelmente da seguinte forma

$$
\frac{n}{\beta} L^{2} e^{-\frac{\alpha 2}{2 m}(q, q)}<1
$$

para algum J sufoientemente grande, porérm independente de $n_{\xi}$ o que năo acontece aqui. 


\subsection{Conclusão}

Vale aqui um cometário sobre a utilização da expansão de cluster para obter estimativas de correlaçāo e provar a transiçāo de fase de Kosterlitz-Thouless em outros modelos além do gás de dipolos.

Primeiramente nos parece razoável repetir os resultados existentes para os gases de Coulomb, a fim de nos assegurarmos do método. É claro que nesses gases também chegaremos a uma expressāo do tipo (3.20) para a função que deverá ser expandida. No capítulo 3 essa função envolvia o termo real $\gamma_{\alpha}(j)$. Nos gases de Coulomb podemos ter funções reais ou imaginárias, dependendo da escolha da própria função de partição ou do operador de desordem para o cálculo do valor esperado da correlação .

A maior diferença, porém, está relacionada com as cargas dos gases de Coulomb, especialmente as do gás de Villain, que podem assumir qualquer valor. Isso afetará as estimativas (3.13), (3.14) que nos dipolos são bem simples por envolver a diferença entre as funçōes de Green calculadas em dois pontos apenas $x$ e $x+L$, onde $L$ é o comprimento do dipolo. Nos gases de Coulomb teremos a interação entre todos os pontos da rede.

Quanto à busca de novos resultados, ainda recentemente ficamos sabendo da proposta de T. Spencer de provar a transição de fase de Kosterlitz-Thouless em uma versão dos modelos s-o-s. A transição de fase no modelo s-o-s foi provada pelo próprio Spencer com os métodos expostos nesta tese. Nessa versão do modelo a função de partição na representação de sine-Gordon a medida é função de $\phi$, mas essa variável é restrita de modo a só poder dar saltos unitários entre sítios. Achamos que a utilização da representação das cargas pode ser proveitosa aqui, visto que na sine-Gordon, nem mesmo Spencer tem uma pista de como proceder. 


\section{Apêndice A}

\section{A Desigualdade Eletrostática}

Obter a desigualdade eletrostática é um simples exercício de completar quadrados no peso de Gibbs. Vamos demonstrá-la para o caso restrito de transformação de cargas. Assim, seja $\mathcal{U}$ um subeonjunto de cargas do găs a que aplicaremos a deslocamento

$$
q_{A}=q_{A}+\frac{1}{8} \Delta\left(\chi_{u} q_{A}\right)
$$

onde

$$
x_{u}=\left\{\begin{array}{l}
1 \text { se } u \in U \\
0 \text { se } u \in U
\end{array}\right.
$$

Por substituição direta

$$
\begin{aligned}
\left(\overline{\mu_{A}}(-\Delta)^{-1} q_{\Lambda}\right) & =\left(q_{\Lambda}+\frac{1}{8} \Delta \chi_{u} q_{\Lambda}(-\Delta)^{-1} q_{\Lambda}+\frac{1}{8} \Delta \chi_{u} q_{\Lambda}\right) \\
& =\left(q_{\Lambda},(-\Delta)^{-1} q_{\Lambda}\right)+\frac{1}{8}\left(\chi_{u} q_{A},-\chi_{u} q_{\Lambda}\right) \\
& +\frac{1}{8}\left(\Delta \chi_{u} q_{\Lambda}(-\Delta)^{-1} \chi_{u} q_{\Lambda}\right)+\frac{1}{64}\left(\Delta \chi_{u} q_{A}, \chi_{u} q_{\Lambda}\right)
\end{aligned}
$$

Agrupando os termos iguais

$$
\left(q_{\Lambda}(-\Delta)^{-1} q_{\Lambda}\right)=\left(q_{A}(-\Delta)^{-1} \bar{\eta}_{\Lambda}\right)+\frac{1}{4}\left(\chi_{u} q_{\Lambda}-\chi_{u} q_{\Lambda}\right)-\frac{1}{64}\left(\Delta \chi_{u} q_{\Lambda}, \chi_{u} q_{\Lambda}\right)
$$

A desigualdade surge quando aproximamos o Laplaciano aplicado à carga pela própria carga, usando que $|\Delta|=8$

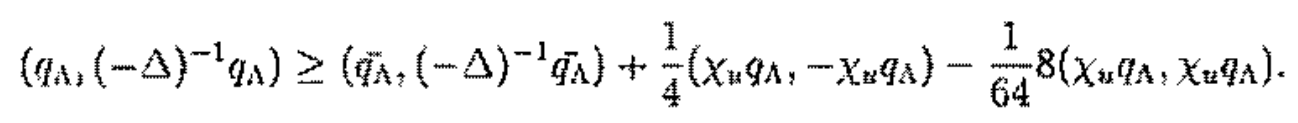


Da substituiçāo no peso de Giblss resulta

$$
\exp \left\{-\frac{\beta}{2}\left(q_{N},(-\Delta)^{-1} q_{n}\right)\right\} \leq \exp \left\{-\frac{\beta}{2}\left(q_{n},(-\Delta)^{-1} \overrightarrow{q_{n}}\right)-\frac{\beta}{16}\left(\chi_{u} q_{n},-\chi_{t} q_{A}\right)\right\}
$$

obtendo o fator de auto-energia

$$
\vec{z}_{y}=z_{y} \exp \left\{-\frac{\beta}{16} \sum_{y \in U} p_{y}^{2}(u)\right\}
$$




\section{Apêndice B}

\section{A Função de Green}

A função de Green (5.11) para os rotores, obtida na seção 5.4, com todos os parâmetros $(N, n, \beta)$ explícitos, foi

$$
C(x, t)=\frac{1}{2 N+1} \frac{1}{\beta} \sum_{p, q} \frac{e^{\imath(p x+q t)}}{\frac{2-2 \cos p}{I}+\frac{2-2 \cos q \delta}{J \delta^{2}}}
$$

$\operatorname{com} \delta=\beta / n \mathrm{e}$

c

$$
p=\frac{2 \pi}{2 N+1} m_{1}, \quad-N \leq m_{1} \leq N
$$

$$
q=\frac{2 \pi}{\beta} m_{2}, \quad-\frac{n}{2} \leq m_{2} \leq \frac{n}{2}
$$

Para obter a função de Green no limite do contínuo, devemos fazer $N \rightarrow \infty$, depois $n \rightarrow \infty$, e por fim tomar $\beta \rightarrow \infty$. A ordem dos dois últimos limites é consequência da fórmula de Trotter.

Quando $N \rightarrow \infty$, (B.1) se torna

$$
C(x, t)=\frac{1}{\beta} \int_{-\pi}^{\pi} \frac{d p}{2 \pi} \sum_{q=-\pi / \delta}^{-\pi / \delta} \frac{e^{t(p x+q t)}}{\frac{2-2 \cos p}{I}+\frac{2-2 \cos \eta \delta}{J \delta^{2}}} .
$$

Em seguida, ao fazermos $n \rightarrow \infty$, o que corresponde a $\delta \rightarrow 0$, e $C(x, t)$ passa a ser dada pela integral

$$
C(x, t)=\frac{1}{\beta} \int_{-\pi}^{\pi} \frac{d p}{2 \pi} \sum_{q=-\infty}^{\infty} \frac{e^{2(p x+q t)}}{\frac{2-2 \cos p}{I}+\frac{q^{2}}{J}}
$$


onde fizemos a aproximação $2-2 \cos q \delta \approx q^{2} \delta^{2}$.

Finalmente tazemos $\beta \rightarrow \infty$. Chegamos a

$$
C(x, t)=\frac{1}{2 \pi^{2}} \int_{-\pi}^{\pi} d p \int_{-\infty}^{\infty} d q \frac{e^{2(p x+q)}}{\frac{2-2 \cos p}{I}+\frac{q^{2}}{J}} .
$$

Nossos resultados dependem da diferença $C(x, t)-C(0,0)$. Vamos calculá-la para o caso particular em que $t=0$. Nesse caso a integral en $q$ pode ser facilmente calculada pela substituição de variảvel $q=\sqrt{(J / I)(2-2 \cos p)} \tan \theta$ :

$$
\int_{-\infty}^{\infty} d q \frac{1}{\frac{2-2 \cos p}{i}+\frac{q^{2}}{J}}=\pi \sqrt{I J} \frac{1}{\sqrt{2-2 \cos p}}
$$

Temos entăo

$$
C(x, 0)-C(0,0)=\frac{1}{4 \pi} \sqrt{I J} \int_{-\pi}^{\pi} d p \frac{e^{\mathrm{x} p x}-1}{\sqrt{2-2 \cos p}} .
$$

É mais conveniente escrever

$$
C(x, 0)-C(0,0)=\frac{1}{2 \pi} \sqrt{I J} \int_{0}^{\pi} d p \frac{\cos p x-1}{\sqrt{2-2 \cos p}}
$$

onde usamos que $2 \cos x=e^{x}+e^{-x}$.

Para calcular a integral (B.3), diwidimos o intervalo de integração em duas partes

$$
C(x, 0)-C(0,0)=\frac{1}{2 \pi} \sqrt{I J} \int_{0}^{1} d p \frac{\cos p-1}{\sqrt{2-2 \cos p}}+\frac{1}{2 \pi} \sqrt{I J} \int_{1}^{\pi} d p \frac{\cos p x-1}{\sqrt{2-2 \cos p}}
$$

A segunda integral pode ser estimada por uma constante independente de $x$. Quanto à primeira integral, no intervalo $[0,1]$ podemos aproximar $2-2 \cos p \approx$ $p^{2}$. Logo,

$$
C(z, 0)-C(0,0) \sim \frac{1}{2 \pi} \sqrt{I J} \int_{0}^{1} d p \frac{\cos p x-1}{p}
$$

A mudanģa de variável $\xi \rightleftharpoons p x$, leva a (para $x>0$ )

$$
C(x, 0)-C(0,0) \times \frac{1}{2 \pi} \sqrt{I J} \int_{0}^{x} d \xi \frac{\cos \xi-1}{\xi}
$$


No intervalo $[0,1)$ expandimos $\cos \xi-1 \approx-(1 / 2) \xi^{2}$. A contribuiçăo dessa regito se integra para uma constante. No intervalo $[1, x]$ usamos integraçăo por partes:

$$
\int_{1}^{x} d \xi \frac{\cos \xi-1}{\xi}=\left.\frac{\sin \xi-\xi}{\xi}\right|_{1} ^{x}+\int_{1}^{x} d \xi \frac{\sin \xi-\xi}{\xi^{2}}
$$

É fácil ver que o valor assintótico é

$$
C(x, 0)-C(0,0) \approx-\sqrt{\frac{I y}{4 \pi^{2}}} \ln |x|
$$

mesmo quando $x<0$. 


\section{Bibliografia}

[1] L. Onsager: "Crystal Statistics I: A Two-Dimensional Model with an Order-Disorder Transition" Phys. Rev. 65, 117 (1944)

[2] F. Spitser: "Principles of Random Walk", van Nostrand, Princeton (1064)

[3] L.Kadanoff: "Scaling Laws for Ising Models near $T_{c}$ " Physics. 2, 263 (1965)

[4] N.D.Mermin, H.Wagner: "Absence of Ferromagnetism or Antiferromagnetism in One- or Two-Dimensional Heisenberg Models." Phys.Rev.Lett. $17,1133(1960)$

[5] H.E.Stanley, T.A.Kaplan: "Possibility of a Phase Transition for the TwoDimensional Heisenberg Model." Phys.Rev.Lett. 17, 913 (1964)

[6] L.P.Kadanoff, W.Gotze, D.Hamblen, R.Hecht, E.A.S.Lewis, V.V.Palciauskas, M.Rayl, J.Swift, D.Aspen, J.Kane: "Statie Phenomena Near Critieal Points: Theory and Experiment" Rew.Mod. Phys. 11967

[7] D.W.Robinson: "A Proof of the Exisitence of Phase Transition in the Anisotropic Heisenberg Model." Commun.Moth. Phys. 14, 195 (1969)

[8] L.P. Kadanof, H. Ceva: "Determination of an Operator Algebra for the Two-Dimensional Ising Model" Phys. Rev. B 3, 3918 (1971)

[9] F. Wegner: "Duality in Generalized Ising Models and Phase Transitions without Order Parameters" J.Math. Phys. 12, 22591971

[10] M. Reed, B. Simon Methods of Modern Mathematical Physics vol. I, Academic Press, NY (1972) 
[11] J.M.Kosterlitz, D.J.Thouless: "Ordering, Metastability and Phase Transition in Two-Dirnensional Systems." J.Phys. C6, 1181 (1973)

[12] J.Villain: "Theory of One- and Two-Dimensional Magnets With An Easy Magnetization Plane". J.Phys. (Paris) 32, 581 (1975)

[13] J.Föhlieh, B.Simon, T.Spencer: "Infrared Bounds, Phase Transition and Continuous Symmetry Breaking." Commun.Moth.Phys, 50, 79 (1976)

[14] T.Blanks, R.Myerson, J.Kogut: "Plase Transition in Abelian Lattice Gauge Theories.", Nucl. Phys. B 129, 493 (1977).

[15] O.A.McBryan, T.Spencer: "On the Decay of Correlations in SO(n)-Syrnmetric Ferromagnets" Commun. Math. Phys. 53, 299 (1977)

[16] F.Dyson, E.H.Lieb, B.Simon: "Phase Transition in Quantum Spin Sysytems with Isotropic and Nonisotropic Interaction." J. Stat. Phys. 18, $335(1978)$

[17] J.Fröhlich, E.H.Lieb: "Phase Transition in Anisotropic Lattice Spin Sys" tems." Commun. Math. Phys. 60, 233 (1978)

[18] V.A.Malyshev: "Phase Transition in Classical Heisenberg Ferromagnets with Arbitrary Parameter of Anisotropy." Commun.Math.Fhys. 40, 75 (1978)

[19] P.M.Bleher, E.Zalys: "Existence of Long-Range Order in the Migdal Recursion Equations" Commun.Math.Phys. 67, 17 (1979)

[20] J.Kogut: "An Introduction to Lattice Gauge Theory and Spin Systems." Rev. Mod. Phys. 51, 659 (1979)

[21] D.Brydges, P.Federbush: "Debye Screening", Commun, Math.Phys. 73 p.197 (1980).

[22] J.Frohlich, T. Spencer: "Phase Diagram and Critical Properties of (Classical) Coulomb Systems" Erie Lectures (1980) 
[23] J.Fröhlich, C. Pfister: "On the Absence of Spontaneous Symmetry Breaking and of Crystalline Ordering in Two-Dimensional Systems" Commun.Math.Phys. 81, 277 (1981)

[24] J.Fröhlich, T.Spencer: "On the Statistical Mechanics of Classical Coulomb and Dipole Gases." J.Stat.Phys. 24, 4 (1981)

[25] J.Fröhlich, T.Spencer: "The Kosterlitz-Thouless Transition in TwoDimensional Abelian Spin Systems and the Coulomb Gas." Commun.Math.Phys. 81, 527 (1981)

[26] J.Glimm and A.Jaffe, "Quantum Physics - A Functional Integral Point of View", Springer-Verlag (1981)

[27] J.Fröhlich, T.Spencer: The Berezinski-Kosterlitz-Thouless Transition (energy-entropy arguments and renormalization in efect gases in Progress in Physics Vol. 7 (1983)

[28] D.Brydges, A Short Course on Cluster Expansions In: Critical Phenomena, Random Systems Gauge Theories. Osterwalder, K. Stora, R. (eds.) Amsterdam: Elsevier, 1986

[29] W.S.Yang: "Debye Screening for Two-Dimensional Coulomb Systems at High Temperature." J.Stat.Phys. 49, 1 (1987)

[30] D.H.U.Marchetti, A.Klein, J.Fernando Perez: "Power-Law Falloff In The Kosterlitz-Thouless Phase Of a Two-Dimensional Lattice Coulomb Gas." J.Stat.Phys. 60, 137 (1990)

[31] J.Dimock, T.R.Hurd: "A Renormalization Group Analysis of the Kosterlitz-Thouless Phase." Comm.Math.Phys. 137, 263

[32] D.H.U.Marchetti, A.Klein: "Power-Law Falloff In Two-Dimensional Coulomb Gases At Inverse Temperature $\beta>8 \pi . "$ J.Stat.Phys. 64, 135 (1991)

[33] A.Klein, J.Fernando Perez: "Localization in the Ground State of a Disordered Array of Quantum Rotators." Commun.Math.Phys. 147, 241 (1992) 
[34] D.H.U.Marchetti: "Upper Bound on the Truncated Connectivity in One-Dimensinal $\beta /|x-y|^{2}$ Percolation Models at $\beta>1$ ". Rev. Math.Phys. 7, 723 (1995) 